\title{
TREHALOSE GLYCOLIPIDS AS IMMUNOMODULATORS AND THEIR INCORPORATION INTO LIPOSOMES FOR DRUG DELIVERY
}

by

Stephanie Houping Chee

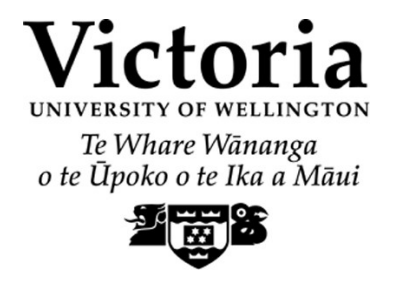

\begin{abstract}
A thesis
submitted to the Victoria University of Wellington in fulfilment of the requirements for the degree of Master of Science in Chemistry
\end{abstract}

Victoria University of Wellington 


\section{ABSTRACT}

The development of novel therapies for cancer and other diseases is an area of enormous research effort due to the growing need for better patient outcomes. As such, not only is the chemical synthesis of new drugs and adjuvants required, but ways to improve drug delivery also need to be explored. Accordingly, there has been much recent effort towards the synthesis and the biological evaluation of bacterial cell wall components as immunomodulatory compounds. To this end, trehalose glycolipids (TGs), which have been isolated from bacteria of the Mycobacteria family, are of significant interest, due to their anti-tumour and adjuvant activities.

In this thesis, the efficient synthesis of trehalose monoesters (TMEs) was investigated and the ability of these monoesters to activate macrophages via Mincle was studied and compared to the activities of their trehalose diester (TDE) counterparts. In this way, a better understanding of how TG structure influences biological activity was explored. Liposomes containing a representative TG (the C26 TDE) were also synthesised, with the objective being to explore whether TGliposomes could be used as improved drug delivery vehicles. To meet these overall objectives, TGs in solution, as well as TG incorporated into liposomes, were tested for their ability to activate macrophages derived from both $\mathrm{C} 57 \mathrm{BL} / 6$ and $\mathrm{Mincle}^{-/-}$ mice, whereby the Mincle receptor is a known receptor for TDEs.

In the TME studies, an optimised synthesis of the monoesters was developed. The ability of the TMEs to active macrophages was explored and, for the first time, it was observed that TMEs have similar biological activities to TDEs. In the TGliposome studies, a variety of liposomes containing different concentrations of phosphatidylcholine (PC) as well as the C26 TDE was prepared, so as to explore how differences in these two constituent parts influence the activation of macrophages. From this work, it was observed that increasing concentrations of TG in the liposome and increasing concentrations of liposomes gave increased 
macrophage activation. A concentration of PC above $200 \mu \mathrm{M}$ also led to macrophage activation, and non-specific cell death was observed at time points $>48$ $\mathrm{h}$ (for the wild type macrophages) and at time points $\geq 48 \mathrm{~h}$ for the Mincle $^{-/-}$ macrophages. Thus, in the case of the TG liposomes, macrophage activation is independent of Mincle, which was unusual as macrophage activation in the case of the individual TGs was dependent on this receptor.

Taken as a whole, these results pave the way for further investigations into utilising TGs in the treatment of diseases. In particular, this work provided insight into the requirement of TG/Mincle binding for improved TGs as potential adjuvants. Moreover, these studies demonstrated that the incorporation of TGs into liposomes leads to enhanced macrophage activation and therefore, potentially enhanced phagocytosis by these immune cells. Accordingly, TG-liposomes may find future application as drug delivery vehicles, in diseases where macrophages play a prominent role. 


\section{PUBLICATION}

The following publication was based upon work carried out during this research project, with the key results discussed in Chapter two of this thesis.

Stocker, B. L.; Khan, A. A.; Chee, S. H.; Kamena, F.; Timmer, M. S. M. 'On One Leg: Trehalose Monoesters Activate Macrophages in a Mincle-Dependent Manner' ChemBioChem 2014, 15, 382. 


\section{ACKNOWLEDGMENTS}

First and foremost, I must thank my supervisors Dr. Bridget Stocker and Dr. Mattie Timmer for their guidance and support. I want to take the time to express my gratitude for your supervision through the course of this project, your patience and commitment to me is very much appreciated. Furthermore, I would like to thank you for fully funding this Master's degree, the financial support you have provided me is much appreciated. I have been blessed to have both of you shape my education.

I wish to extend my thanks to Dr. Jacquie Harper and the Malaghan Institute of Medical Research for allowing me to conduct my 'bio experiments' in their specialised facilities, and for giving me the opportunity to make the transition from chemistry to immunology. Additionally, I would like to thank the Immunoglycomics group, as well as the Arthritis and Inflammation group; it has been a pleasure to work alongside all of you in the lab. To the staff and fellow postgraduate students here in the School of Chemical and Physical Sciences at VUW, I extend my thanks to you all. To my friends outside of the University, thank you for your friendship, kindness and understanding.

Finally, I wish to thank my family. Thank you to Mum, Dad and James for your love, support and encouragement. 


\section{TABLE OF CONTENTS}

Acknowledgments $\quad$ v

List of Figures viii

List of Schemes $\quad$ ix

List of Tables $\quad x$

List of Abbreviations $\quad$ xi

$\begin{array}{lr}\text { Chapter 1: Background } & 15\end{array}$

$\begin{array}{lll}1.1 & \text { Mycobacteria in disease } & 15\end{array}$

1.2 Trehalose Glycolipids: The Trehalose 6,6'-diester family 17

1.3 The TDM Receptor: Macrophage Inducible C-type Lectin (Mincle) 28

$\begin{array}{lll}1.4 & \text { Liposomes } & 34\end{array}$

1.5 Macrophages in disease 40

$\begin{array}{lll}1.6 & \text { Research aims } & 42\end{array}$

Chapter 2: Synthesis and Biological Evaluation of C26-TME 43

2.1 Overview 43

2.2 Synthesis of TDE 43

$\begin{array}{lll}2.3 & \text { Synthesis of TME } & 47\end{array}$

2.4 Biological evaluation of TMEs $\quad 57$

$\begin{array}{lll}2.5 & \text { Conclusion } & 64\end{array}$

Chapter 3: Formation and Biological Evaluation of TGincorporated Liposomes $\quad 65$

$\begin{array}{lll}3.1 & \text { Overview } & 65\end{array}$ 
3.3 Characterisation of Liposomes: Percentage of C26 TDE incorporated into liposomes

3.4 Characterisation of Liposomes: Size and distribution

3.5 Ability of Liposomes to Activate Macrophages

3.6 Conclusion

Chapter 4: Conclusions and Future prospects

4.1 Conclusions

4.2 Future Prospects

Chapter 5: Experimental Procedures 93

References

103 


\section{LIST OF FIGURES}

Figure 1. The family of trehalose glycolipids............................................... 19

Figure 2. The Th-1/Th-17 immune response by Mincle ................................ 30

Figure 3. Mincle-trehalose disaccharide interactions at both the primary and

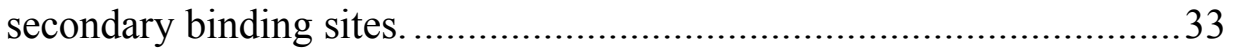

Figure 4. ${ }^{1} \mathrm{H}$ NMR spectra of TMS-protected TDE 25.....................................55

Figure 5. ${ }^{1} \mathrm{H}$ NMR spectra of TMS-protected TME 31 ...................................56

Figure 6. Schematic for Bone Marrow Isolation and BMM Differentiation.........58

Figure 7. Production of NO by TDB/TME-treated BMMs. ............................ 61

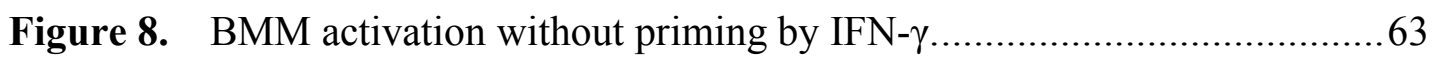

Figure 9. Standard curve of $\alpha, \alpha^{\prime}$-trehalose in distilled water...........................69

Figure 10. Titration of $\mathrm{PC} /$ cholesterol liposomes in BMMs.............................73

Figure 11. Cartoon representing the three different formulations of C26 TDE incorporated liposomes that were prepared....................................... 78

Figure 12. The liposomes containing a $6.3 \%$ weight loading of the C26 TDE for the initial $24 \mu \mathrm{M}$ solution, which was then serially diluted to $12 \mu \mathrm{M}$ and $6 \mu \mathrm{M}$.

Figure 13. Titration of C26 TDE liposomes (6.3 weight \%) in C57BL/6 BMMs. 80

Figure 14 Titration of C26 TDE liposomes (6.3 weight \%) in Mincle ${ }^{-/-}$BMMs...82

Figure 15. The liposomes containing a weight loading of $6.3,2.3$ and $0.5 \%$ of C26 TDE.

Figure 16. Activation of BMMs from C57BL/6 by C26-TDE liposomes and C26 TDE solutions. 


\section{LIST OF SCHEMES}

Scheme 1. Synthesis of the $\alpha$-alkyl- $\beta$-hydroxy fragment..................................23

Scheme 2. Synthesis of the meromycolate branch of the mycolic acid...................25

Scheme 3. Synthesis of $\alpha$-TDM 22 and TMM 23 ...........................................27

Scheme 4. Synthesis of TDE 26 (C26-diester) .................................................44

Scheme 5. Reaction mechanism for BSA mediated silylation of trehalose. ${ }^{28}$.........46

Scheme 6. Chemoenzymatic synthesis of mono-acylated trehalose derivatives 29

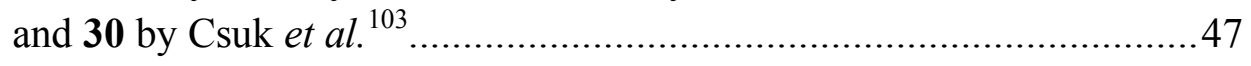

Scheme 7. Synthesis of TME 32 (C26-monoester) ............................................50

Scheme 8. Mechanism for the formation of $N$-hexacosanoyl urea side-product and

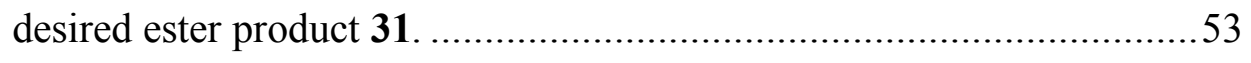

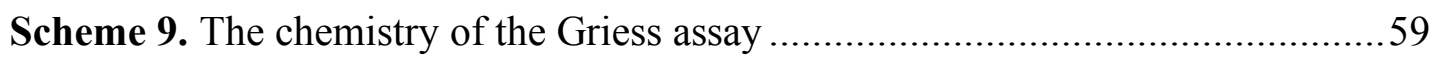

Scheme 10. The mechanism of the phenol-sulfuric acid colourimetric assay. .........68 


\section{LIST OF TABLES}

Table 1. Optimisation of synthesis of mono-ester 31 ...................................51

Table 2. Percentage of C26-TDE incorporated into liposome stock suspensions. .. 70 


\section{LIST OF ABBREVIATIONS}

\begin{tabular}{|c|c|}
\hline ACAT & acyl-CoA:cholesterol acyltransferase \\
\hline Apa & alanine proline antigen \\
\hline APC & antigen presenting cell \\
\hline BCG & Bacillus Calmette-Guérin \\
\hline $\mathrm{BMM}$ & bone marrow-derived macrophage \\
\hline BODIPY & boron-dipyrromethene \\
\hline BSA & $\mathrm{N}, \mathrm{O}$-bis(trimethylsilyl)acetamide \\
\hline CAS & chemical abstracts service \\
\hline $\mathrm{CD}$ & cluster of differentiation \\
\hline CRD & carbohydrate recognition domain \\
\hline $\mathrm{DC}$ & dendritic cell \\
\hline DCP & dicetylphosphate \\
\hline DDA & dimethyl dioctadecylammonium \\
\hline DEAD & diethyl azodicarboxylate \\
\hline DIAD & diisopropyl azodicarboxylate \\
\hline DLS & dynamic light scattering \\
\hline DMAP & 4-dimethylaminopyridine \\
\hline $\mathrm{DMF}$ & dimethylformamide \\
\hline DMSO & dimethyl sulfoxide \\
\hline DNA & deoxyribonucleic acid \\
\hline DSPE & distearoylphosphatidylethanolamine \\
\hline $\mathrm{E} 1 \mathrm{cB}$ & unimolecular elimination conjugate base \\
\hline EDCI & 1-ethyl-3-(3-dimethylaminopropyl)carbodiimide \\
\hline equiv. & equivalent \\
\hline FCA & Freund's complete adjuvant \\
\hline FDA & food and drug administration \\
\hline
\end{tabular}




\begin{tabular}{|c|c|}
\hline GM-CSF & granulocyte macrophage colony-stimulating factor \\
\hline HIV-1 & human immunodeficiency virus type 1 \\
\hline HMPA & hexamethylphosphoramide \\
\hline i.t. & intratracheal \\
\hline i.v. & intravenous \\
\hline IFN & interferon \\
\hline IL & interleukin \\
\hline iNOS & inducible nitric oxide synthase \\
\hline LAL & limulus amebocyte lysate \\
\hline LDA & lithium diisopropylamide \\
\hline LiHMDS & lithium bis(trimethylsilyl)amide \\
\hline LPS & lipopolysaccharide \\
\hline LUV & large unilamellar vesicle \\
\hline $\mathrm{M \varphi}$ & macrophage \\
\hline M. $t b$ & Mycobacterium tuberculosis \\
\hline $\mathrm{MCP}$ & monocyte chemoattractant protein \\
\hline$m \mathrm{CPBA}$ & meta-chloroperoxybenzoic acid \\
\hline MFI & mean fluorescence intensity \\
\hline $\mathrm{MHC}$ & major histocompatibility complex \\
\hline Mincle & macrophage inducible C-type lectin \\
\hline MIP & macrophage inflammatory protein \\
\hline MLV & multilamellar vesicle \\
\hline MP & melting point \\
\hline MTT & 3-(4,5-dimethylthiazol-2-yl)-2,5-diphenyltetrazolium bromide \\
\hline NK & natural killer \\
\hline NMR & nuclear magnetic resonance \\
\hline $\mathrm{NO}$ & nitric oxide \\
\hline NOD & nucleotide binding oligomerisation domain \\
\hline OVA & ovalbumin \\
\hline PAMP & pathogen associated molecular pattern \\
\hline
\end{tabular}




\begin{tabular}{|c|c|}
\hline PBS & phosphate-buffered saline \\
\hline $\mathrm{PC}$ & phosphatidylcholine \\
\hline PCC & pyridinium chlorochromate \\
\hline PEG & polyethylene glycol \\
\hline PG & phosphatidylglycerol \\
\hline PRR & pattern recognition receptor \\
\hline PS & phosphatidylserine \\
\hline RPM & revolutions per minute \\
\hline SEM & standard errors of the mean \\
\hline SPR & surface plasmon resonance \\
\hline SUV & small unilamellar vesicle \\
\hline TAM & tumour-associated macrophage \\
\hline TB & tuberculosis \\
\hline TBAF & tetra-butylammonium fluoride \\
\hline TBDPS & tert-Butyldiphenylsilyl \\
\hline TBTU & tetra-methyluronium tetrafluoroborate \\
\hline $\mathrm{T}_{c}$ & transition temperature \\
\hline TDB & trehalose dibehenate \\
\hline TDCM & trehalose dicorynomycolate \\
\hline TDE & trehalose diester \\
\hline TDM & trehalose dimycolate \\
\hline TG & trehalose glycolipid \\
\hline TGF & transforming growth factor \\
\hline $\mathrm{Th}$ & T helper \\
\hline THF & tetrahydrofuran \\
\hline THP & tetrahydropyranyl \\
\hline TLC & thin-layer chromatography \\
\hline TLR & toll-like receptors \\
\hline TME & trehalose monoester \\
\hline TMM & trehalose monomycolate \\
\hline
\end{tabular}


TMS trimethylsilyl

TNF tumour necrosis factor 


\section{CHAPTER 1:}

\section{BACKGROUND}

\subsection{Mycobacteria in disease}

The interactions between humans and bacteria are among the most diverse investigative fields in the medical sciences. Although chronic infections due to bacteria have contributed to their negative image, there are also many bacteria which can be beneficial to humans, such as intestinal microflora and commensal microbes found on the body's surface. ${ }^{1}$ As the presence of bacteria often stimulates the immune system, the use of bacteria in the treatment of diseases has also been explored.

Before there was much knowledge and understanding of how the immune system acts on tumours, a few medical doctors reported that infection could cause tumour regression. ${ }^{2}$ These observations date back to the 1800's when German physicians W. Busch and F. Fehleisen separately noticed tumour regression in cancer patients who were infected by erysipelas. Fehleisen later determined that erysipelas is caused by Streptococcus pyrogenes. ${ }^{1}$

A short time later, a New York surgeon, William Coley, observed that malignant tumours regressed in patients who were suffering from bacterial infections. ${ }^{3}$ Coley treated bone and soft tissue sarcoma patients with erysipelas and noted infectionassociated tumour regression. ${ }^{1}$ He then experimented with treatments involving live Streptococci and while patient outcomes were encouraging, systemic Streptococcus infection was fatal. Subsequently, Coley used heat-killed Streptococcus organisms with heat-killed Serratia marcescens, which became a bacterial vaccine known as "Coley's toxin". ${ }^{4}$ Over the next 40 years, Coley treated more than 1000 cancer patients with 
bacteria or bacterial products, which led to a high success rate in the treatment of sarcomas and other malignancies, including carcinomas (e.g. breast and renal cancer), lymphomas and melanomas. However, his work was criticised as his results could not be reproduced due to poor documentation and the follow-up of patients, and inconsistencies in the preparation of the toxins. There were thirteen different toxin preparation methods reported, which all varied in effectiveness. With the emergence of chemotherapy and radiotherapy, Coley's work thus gradually fell out of favour.

Today, however, it is well established that bacteria, as well as bacterial components, can activate the immune system ${ }^{5}$ and can be used in the treatment of disease. There are several examples of attenuated bacteria that have been used in vaccines. For example, Salmonella typhi is used in the Ty21a vaccine to vaccinate against typhoid fever, ${ }^{6}$ Vibrio cholerae is used in CVD $103-\mathrm{HgR}$ to vaccinate against cholera, ${ }^{6}$ and Mycobacterium tuberculosis in Calmette-Guérin (BCG) for vaccination against tuberculosis. ${ }^{7}$ Another prominent example is the use of attenuated Mycobacterium tuberculosis $(M$. tb), which forms a key component in Freund's complete adjuvant (FCA) system. FCA is effective in stimulating cell-mediated immunity and has thus been used in a number of animal models of disease. FCA has also been found to have potential in the prevention of juvenile-onset diabetes in mouse models, ${ }^{8}$ as well as in Parkinson's disease in rat models, ${ }^{9}$ however, the adjuvant cannot yet be applied in human studies due to issues with toxicity. ${ }^{10}$ To this end, there has been much interest in determining how the individual components of the mycobacterial cell wall influence the immune response.

Of particular interest to my Masters research is exploring the potential of trehalose glycolipids in the treatment of disease. Complex trehalose glycolipids, the trehalose dimycolates (TDMs), are found in the outer cell wall of $M$. $t b$ and related mycobacteria and have been found to be highly immunostimulatory. Indeed, the Th1 and Th17 immune response by FCA has recently been attributed to TDM and peptidoglycan, ${ }^{11}$ while the CAF01 adjuvant system, which consists of a cationic liposome containing a synthetic trehalose glycolipid (trehalose dibehenate, TDB) and a recombinant $M$. Tb 
fusion protein has shown promise in the treatment of tuberculosis. ${ }^{12} \mathrm{TDM}$ is the most abundant lipid produced by virulent $M$. $t b$, yet despite this, the mechanism of activity of the glycolipid in the context of tuberculosis pathogenicity still remains puzzling. This is due in part to the glycolipid's ability to change from non-toxic to highly toxic depending on the aggregation state of the glycolipids. ${ }^{13}$ The exact structure of the glycolipid has also been found to affect the specific immune reponse. ${ }^{13}$

\subsection{Trehalose Glycolipids: The Trehalose 6,6'-diester family}

Trehalose glycolipids were initially discovered as an important factor in the mycobacterial cell wall that contributes to the bacteria's virulence. ${ }^{14}$ In the early 1950 s, Bloch found that virulent strains of mycobacteria contained a material called "cord factor," which was essential for the pathogenesis of mycobacteria. ${ }^{15}$ In subsequent years, cord factor was found to be a class of compounds called trehalose [ $\alpha$-D-glucosyl- $(1 \rightarrow 1)$ $\alpha$-D-glucose] dimycolates. ${ }^{16,17}$ A variety of trehalose glycolipids have subsequently been isolated from bacteria such as Mycobacteria and Corynebacteria, and many of these glycolipids exhibit important and often different biological properties. ${ }^{14}$

The family of trehalose glycolipids (Figure 1) is divided into two classes: 6,6'-trehalose diesters [including fatty acid esters (TDEs, $\mathbf{1}$ ), trehalose dicorynomycolates (TDCMs, 2) and trehalose dimycolates (TDMs, 3)] and 2,3-trehalose diesters. ${ }^{14}$ TDMs are the most widely studied of the trehalose glycolipids, and are characterised by the presence of a mycolic acid and a trehalose moiety. ${ }^{14}$ The mycolic acid contains at least two chiral centres ( $\alpha$ - and $\beta$ - to the carboxylic acid), both with $R$-configuration. ${ }^{18}$ In mycolic acids (4), the lipid chain positioned $\alpha$ - to the carboxylate is called the alpha- $(\alpha-)$ branch, while the other portion is known as the meromycolate branch. In different TDMs, only the $\alpha$-branch differs in the number of carbon atoms, however, the meromycolate is more variable and contains different functional groups. ${ }^{19}$ These differences allow mycolic acids to be classified into two groups: the $\alpha$-mycolates, which do not contain 
oxygenated functional groups in the meromycolate branch, and the oxygenated mycolates. The most common $\alpha$-mycolates are those which have cis cyclopropane groups on the meromycolate branch $(\mathbf{4 A})$, while for the oxygenated meromycolates, those containing keto- $(\mathbf{4 B})$ or epoxy-groups $(\mathbf{4 C})$ are the most common. ${ }^{20}$

Today, there are still an extraordinary number of novel trehalose glycolipids being discovered, including maradolipids, a much shorter glycolipid found in the dauer larvae of C. elegans in $2010 .^{21}$ The sheer wealth of trehalose glycolipids found within biological systems, however, has its disadvantages. For example, TDM extracts from mycobacteria alone contain over 500 different compounds which differ in chain length and functional groups (e.g. ketones, cyclopropanes, methoxy groups, Figure 1). ${ }^{21}$ As a consequence, the immunological profile of distinct compounds is difficult to discern. In view of this, much effort has been placed on obtaining homogenous materials through chemical synthesis so that the biological profile of each individual glycolipid can be better understood. 

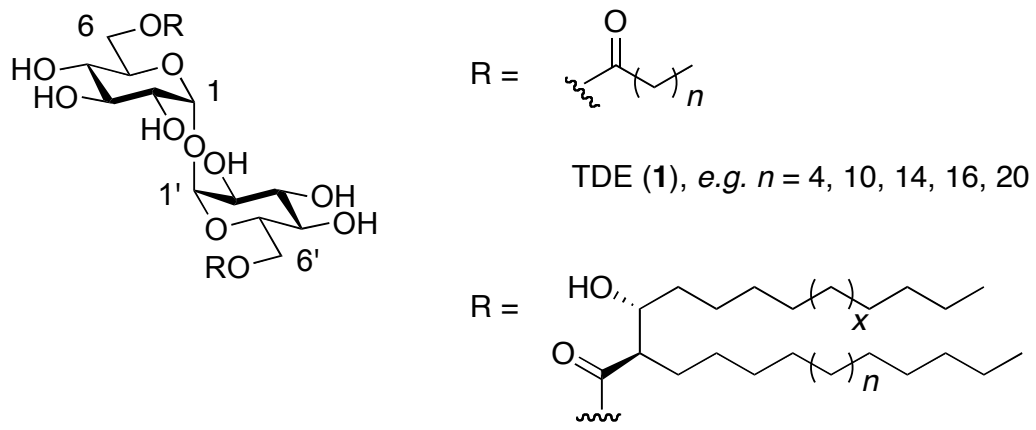

$\operatorname{TDCM}(2)$, e.g. $n=5, x=7$

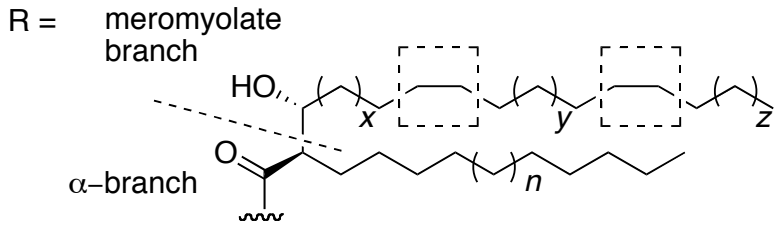

$\operatorname{TDM}(3)$, e.g. $n=13, x=16, y=12, z=17$

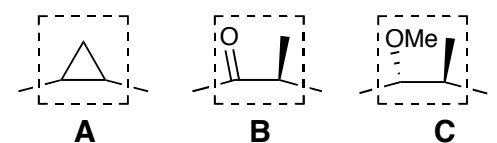

Mycolic acids (4), $x, y, z$ : total chain length is $\mathrm{C}_{60}-\mathrm{C}_{90}, n=13-19$

Figure 1. The family of trehalose glycolipids.

\subsubsection{Synthesis of Trehalose diesters (TDEs)}

The simplest glycolipids found in the trehalose $6,6^{\prime}$-diester family are trehalose diesters of fatty acids (TDEs, e.g. 1, Figure 1). TDEs are differentiated by their lipid length in the $\alpha$ branch of the mycolic acid. It is only in recent years that these compounds have been discovered in the animal kingdom, ${ }^{21}$ although they have been synthesised and

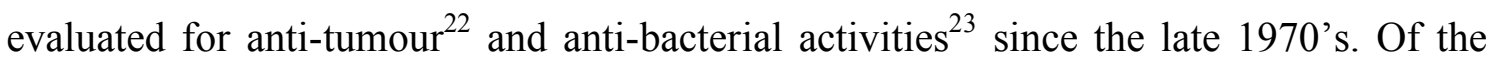
TDEs, the most well-studied is the C22 derivative, trehalose dibehenate (TDB, $n=20$, 
Figure 1), which has been found to bind and activate macrophages $(\mathrm{M} \varphi)$ in the same way as the more complex TDMs. ${ }^{11}$

Several different approaches have been developed over time for the synthesis of TDEs. One of the first synthetic methods, reported in 1973 by Toubiana et al., involved the use of a potassium carbonate mediated trans-esterification of fatty acid methyl esters with trehalose. ${ }^{24}$ Although this reaction successfully allowed for the formation of esters, it proved unselective and mixtures of mono-, di- and tri-esters were also formed. Others have reported on the reaction of $6,6^{\prime}$-di-O-tosyl-trehalose with the potassium salt of palmitic acid, ${ }^{25}$ though a major drawback of this methodology is the formation of 3,6anhydro-trehalose products and other by-products that are hard to separate from the desired TDE. This methodology was later improved by Toccane ${ }^{26}$ and Toubiana et al. ${ }^{32}$ who proposed the use of another starting material, 6,6'-dihalo-6,6'-dideoxy-trehalose, for the synthesis of TDEs to eliminate the occurrence of these anhydro-trehalose side products. It has also been suggested that the esterification reaction en route to the synthesis of TDEs can be improved through the use of benzyl-protected trehalose $\left(2,2^{\prime}, 3,3^{\prime}, 4,4^{\prime}\right.$-hexa-O-benzyl-trehalose $)$ as an intermediate, ${ }^{27}$ however, our group observed that subsequent removal of the benzyl groups from glycolipids with a fatty acid chain of more than 18 carbon atoms was difficult. ${ }^{28}$ Here, hydrogenation proved to be sluggish, which has also been reported previously, ${ }^{29}$ and was likely due to the poor solubility of these amphiphilic compounds. The reaction of trehalose with fatty acids in the presence of diisopropyl azodicarboxylate (DIAD) and triphenyl phosphine $\left(\mathrm{PPh}_{3}\right)$ has also been reported for the synthesis of TDEs. ${ }^{30}$ Here, unprotected trehalose and palmitic acid were subjected to a Mitsunubo reaction to give 6,6'-trehalose dipalmitate in $59 \%$ yield. $^{30}$ In later studies, the scope of this methodology was extended to the synthesis of more complex TDMs. ${ }^{31}$

The use of TMS-protected trehalose $\left(2,2^{\prime}, 3,3^{\prime}, 4,4^{\prime}\right.$-hexa-O-trimethylsilyl-trehalose $)$ has proven to be a useful intermediate in the synthesis of a variety of TDEs. TMS-protected trehalose is appealing because of the ease with which the TMS groups can be removed 
after esterification. Toubiana and co-workers initially synthesised hexa-trimethylsilyl trehalose in two steps from commercially available trehalose, ${ }^{32}$ and later Johnson developed a two-step one-pot synthesis of this key intermediate, ${ }^{33}$ which was subsequently used for the formation of trehalose dipalmitate. ${ }^{34}$ Datta et al. have also used similar methodology, whereby the amount of carboxylic acid was manipulated to give both the mono- and di-esters in good yields, ${ }^{35}$ while Khan et al. used this intermediate in the synthesis of TDEs of various lipid lengths. ${ }^{28}$

The chemoenzymatic synthesis of fatty acid mono- and di-esters of trehalose has also been explored. There is great interest in chemoenzymatic syntheses due to the ability to regio- and stereo-selectively transform saccharides. The most common enzyme used in trehalose acylation is a lipase from Candida antarctia. Oosterom et al. reported the use of this lipase for the synthesis of the 6-butanoyl ester of trehalose in tert-butyl alcohol ${ }^{36}$ and Part et al. have also used this lipase for yielding trehalose 6,6'-vinyladipoyl diesters. ${ }^{37}$ All enzymatic TDE synthesis to date, however, have contained lipids that are short in length.

\subsubsection{Biological activity of TDEs}

TDEs have been found to exhibit a number of interesting biological properties, including anti-cancer activity and an ability to confer protection against pathogenic challenge. ${ }^{14,38,39}$ That said, there is limited knowledge about the underlying cause of the stated biological effects, which in part is due to inconsistencies across the various experimental models. In the case of the anti-cancer activity of TDEs, it is not well understood how the structure of the TDE affects their tumoricidal properties, though it was observed that at least 10 carbons on the acyl chain were required to induce antitumour activity in Ehlrich ascites carcinoma. ${ }^{22}$ Moreover, the addition of lipopolysaccharide (LPS), a major component of the outer membrane of Gram-negative bacteria, has been found to enhance the anti-tumour activities of linear TDEs in in vivo 
tumour studies, ${ }^{40}$ and the presence of an oil-vehicle also appears to enhance the antitumour response. ${ }^{41}$ No insight was provided as to why oil/water emulsions increase the anti-tumour activity of TDEs, though later studies with TDMs has led to the hypothesis that TDMs in oil/water leads to the formation of monolayers, and it is these monolayers that are cytotoxic. ${ }^{42}$ With regard to the ability of TDEs to confer protection against pathogenic organisms, neither the C16 and C22 linear TDEs conferred protection against Klebsiella pneumoniae and Listeria monocytogenes, ${ }^{23}$ however, the C16 TDE did confer protection when tested on Schistosoma mansoni, ${ }^{38}$ and Salmonella typhi and Salmonella typhimurium. ${ }^{39}$

In an attempt to better understand the biological activities of TDEs, and in particular how the TDE lipid length affects the innate immune response of M $\varphi s$, Khan et al. explored the ability of a series of TDEs to activate Bone marrow derived macrophages (BMMs). Here, BMMs were stimulated by TDEs varying in lipid chain length (C4-C26) and the supernatant was analysed for NO, interleukin (IL)- 6 and IL-1 $\beta$ production. From this work it was demonstrated that a lipid length of more than 18 carbon atoms was required for $\mathrm{M} \varphi$ activation. ${ }^{28}$

\subsubsection{Synthesis of Trehalose Dimycolates (TDMs)}

The complexity of TDMs has made their synthesis challenging and to date, only a few groups have embarked upon this endeavour. Baird and co-workers have been leaders in this field and have focused on the synthesis of various mycolic acids en route towards the synthesis of TDMs, ${ }^{43-45}$ and more recently, have synthesised individual TDMs. ${ }^{46}$ To synthesise the mycolic acid portion of TDM, Baird and co-workers used a variety of different approaches, however, a key strategy in their overall route included a FráterSeebach alkylation for the instalment of the $\alpha$-branch of the mycolic acid. In their first synthesis of the $\alpha$-mycolic acid, Baird and co-workers reported a low yield of $31 \%$ for their Fráter-Seebach alkylation when using tetracosanyl iodide $\left(\mathbf{5} \rightarrow \mathbf{6}\right.$, Scheme 1), ${ }^{43}$ 
while in later syntheses, the group developed a chain-elongation strategy ${ }^{47}$ involving an $\alpha$-alkylation using allyl iodide, followed by ozonolysis and a Julia-Kocienski reaction. To complete the synthesis of the $\alpha$-alkyl- $\beta$-hydroxy fragment, ${ }^{43}$ the secondary alcohol of the Fráter-Seebach alkylation product $\mathbf{6}$ was protected with an acetate group, the tertbutyldiphenylsilyl (TBDPS) group was deprotected, and the resulting primary hydroxyl oxidised to give aldehyde 7, the core fragment of the target mycolic acid. More recently, Khan et al. reported on the use of long chain allylic iodides in Fráter-Seebach alkylations, which allowed for improved yields for the $\alpha$-alkylation step. ${ }^{47}$

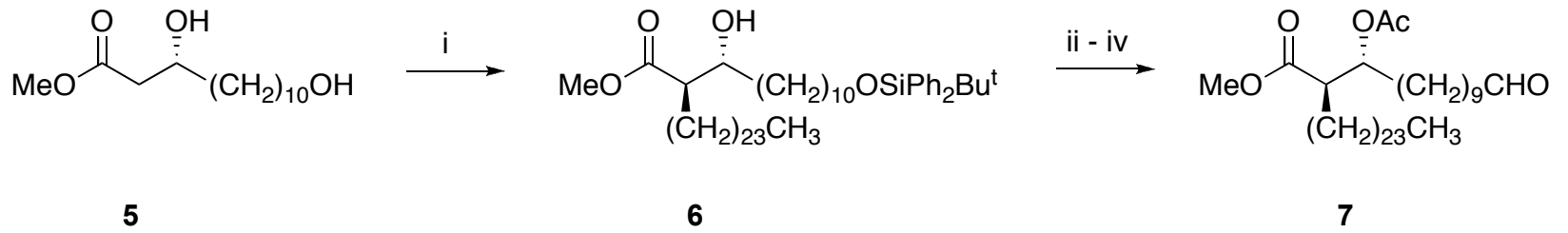

Scheme 1. Synthesis of the $\alpha$-alkyl- $\beta$-hydroxy fragment; Reagents and conditions: (i) $\mathrm{LDA}, \mathrm{CH}_{3}\left(\mathrm{CH}_{2}\right)_{23} \mathrm{I}, \mathrm{HMPA}, 31 \%$; (ii) $\mathrm{Ac}_{2} \mathrm{O}$, pyridine, $86 \%$; (iii) TBAF, 75\%; (iv) PCC, 95\%.

For the synthesis of the meromycolate branch of the mycolic acid, Baird and co-workers employed a number of different strategies depending on the functional groups to be incorporated. ${ }^{45,48-50} \mathrm{~A}$ representative example of the synthesis of an $\alpha$-mycolate is given in Scheme 2. Here, aldehyde $\mathbf{8}$ was subjected to a Wittig reaction with $\mathrm{MeO}_{2} \mathrm{C}\left(\mathrm{CH}_{2}\right)_{10} \mathrm{CH}=\mathrm{PPh}_{3}$, followed by reduction of the resulting ester to the alcohol, hydrogenation of the double bond and oxidation of the alcohol to give aldehyde $9 .{ }^{51}$ Reaction of ester $\mathbf{1 0}$ with thiazole $\mathbf{1 1}$ and subsequent oxidation of the resulting thioether then gave sulfone 12, which was reacted with aldehyde 9 in a Julia-Kocienski reaction to give alkene $\mathbf{1 3}$ as a mixture of $E$ and $Z$ isomers. Reduction of ester $\mathbf{1 3}$ using lithium aluminium hydride $\left(\mathrm{LiAlH}_{4}\right)$, followed by hydrogenation of the alkene gave the dicyclopropyl-alcohol 14. Reaction of alcohol 14 with thiazole 11, followed by oxidation of the resulting thioether led to sulfone 15, which was then reacted 
with aldehyde 7 (from Scheme 1) in a further Julia-Kocienski reaction to form methyl ester 16. Finally, hydrogenation of the alkene in 16, followed by hydrolysis of the acetate and methyl esters led to the $\alpha$-mycolic acid $\mathbf{1 7}$. 

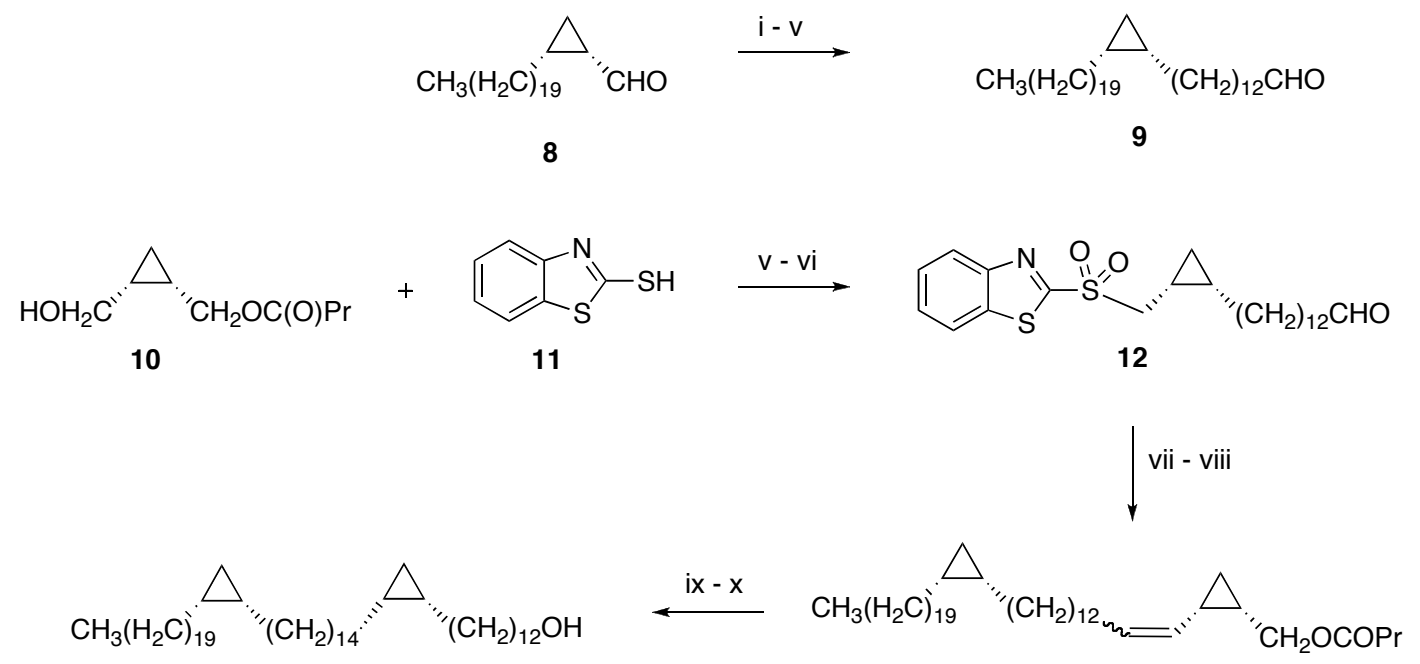

14

13

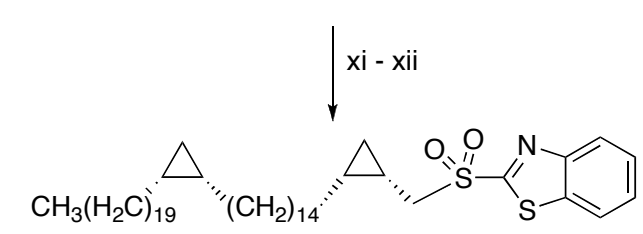

15

xiii

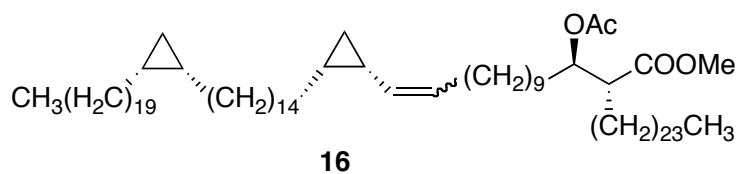

xiv - xv

$\mathrm{CH}_{3}\left(\mathrm{H}_{2} \mathrm{C}\right)_{19}^{\Delta " \prime\left(\mathrm{CH}_{2}\right)_{14}} \triangle_{17}^{\triangle}\left(\mathrm{CH}_{2}\right)_{11} \underbrace{\mathrm{OH}}_{\left(\overline{\bar{C}} \mathrm{H}_{2}\right)_{23} \mathrm{CH}_{3}} \mathrm{CO}_{2} \mathrm{H}$

Scheme 2. Synthesis of the meromycolate branch of the mycolic acid; Reagents and conditions: (i) $\mathrm{MeO}_{2} \mathrm{C}\left(\mathrm{CH}_{2}\right)_{11} \mathrm{PPh}_{3} \mathrm{I}, \mathrm{NaOMe}, \mathrm{DMF}, 70 \%$; (ii) $\mathrm{LiAlH}_{4}$, THF, 92\%; (iii) $\mathrm{N}_{2} \mathrm{H}_{4}, \mathrm{NaIO}_{4}, \mathrm{AcOH}, \mathrm{CuSO}_{4}, i$-PrOH, $85 \%$; (iv) $\mathrm{PCC}$, $\mathrm{CH}_{2} \mathrm{Cl}_{2}, 93 \%$; (v) $\mathrm{PPh}_{3}, \mathrm{DEAD}, 77 \%$; (vi) $m \mathrm{CPBA}, \mathrm{CH}_{2} \mathrm{Cl}_{2}, 82 \%$; (vii) LiHMDS; (viii) 9, 43\% over two steps; (ix) $\mathrm{LiAlH}_{4}$; (x) $\mathrm{NH}_{2} \mathrm{NH}_{2}, \mathrm{NaIO}_{4}$, $\mathrm{CuSO}_{4}$, AcOH, $i$-PrOH, 77\% over two steps; (xi) 11, $\mathrm{PPh}_{3}, \mathrm{DEAD}, 66 \%$; (xii) $m \mathrm{CPBA}, \mathrm{CH}_{2} \mathrm{Cl}_{2}, 62 \%$; (xiii) LiHMDS, then 7, 37\%; (xiv) dipotassium azodicarboxylate, $\mathrm{CH}_{3} \mathrm{COOH}$, THF, 60\%; (xv) LiOH, THF, $\mathrm{H}_{2} \mathrm{O}, \mathrm{MeOH}, 45^{\circ} \mathrm{C}$. 
Baird and co-workers then used this mycolic acid to prepare an $\alpha$-TDM (Scheme 3). The hydroxyl group of the mycolic acid was first protected as a TBDMS ether $(\rightarrow \mathbf{1 8})$ and 18 was subsequently coupled to hexa-trimethylsilyl-trehalose 19. Using this approach, both di-esterified and mono-esterified products were obtained in good yield, and these were then deprotected in two steps to obtain TDM 22 and trehalose monomycolate (TMM) $23{ }^{46}$ 

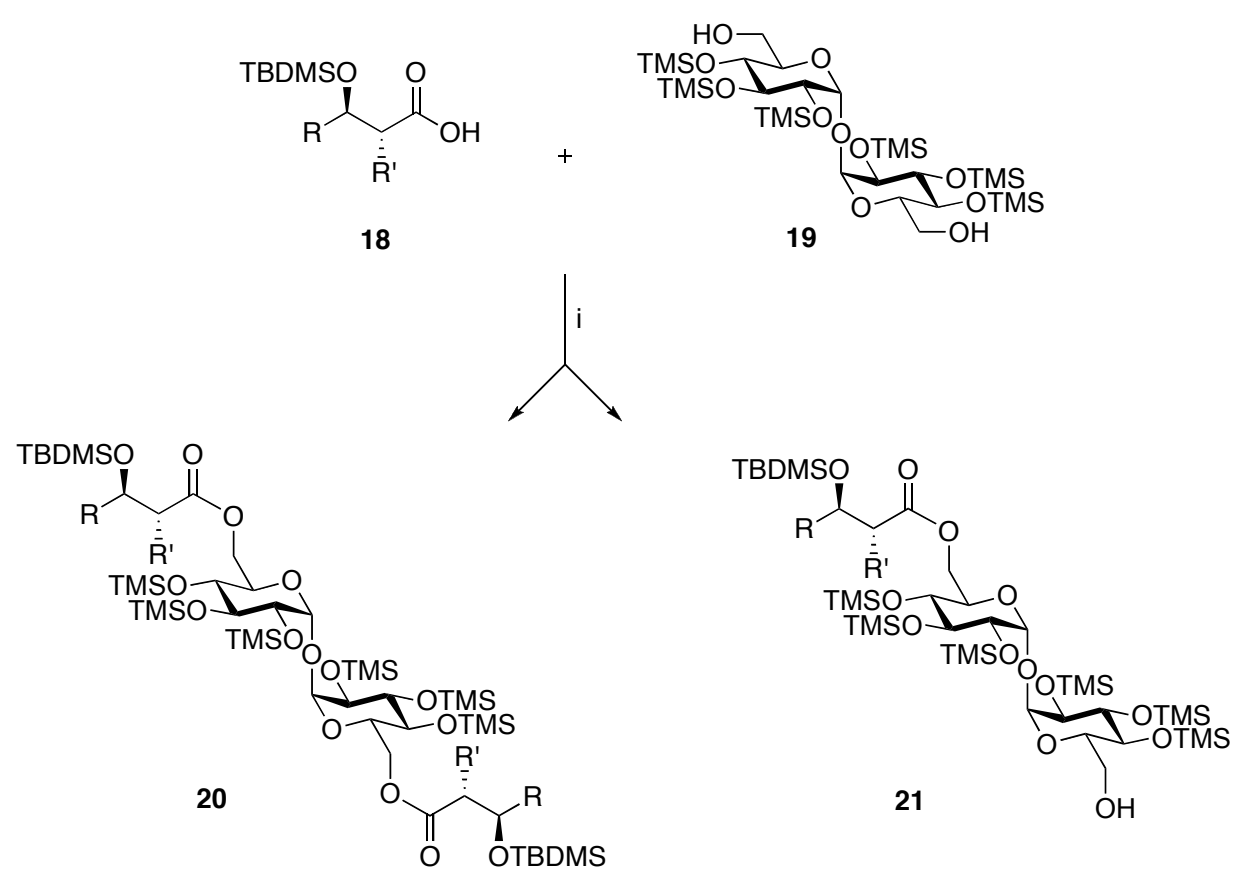

$$
\downarrow \text { ii, iii }
$$$$
\downarrow \text { ii, iii }
$$
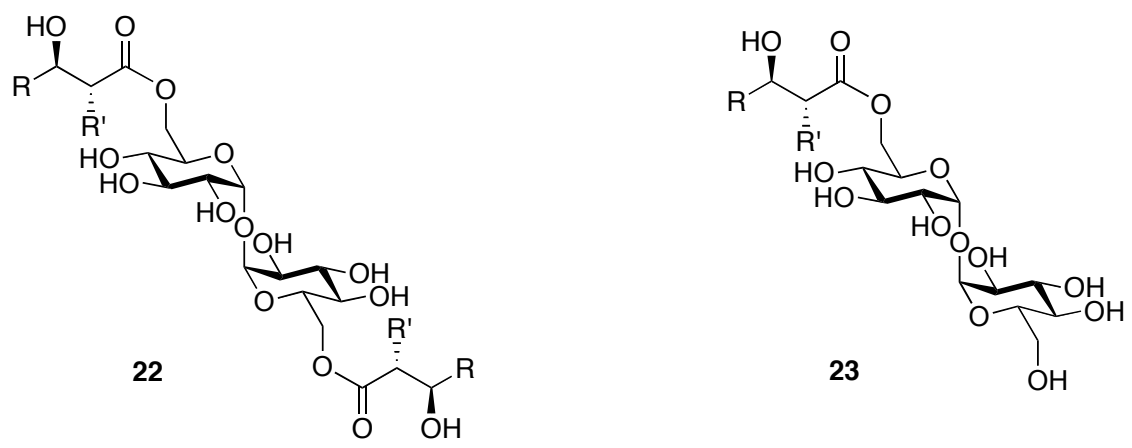

$$
\mathrm{R}^{\prime}=-\left(\mathrm{CH}_{2}\right)_{23} \mathrm{CH}_{3}
$$

Scheme 3. Synthesis of $\alpha$-TDM 22 and TMM 23; Reagents and conditions: (i) EDCI, 4-DMAP, $\mathrm{CH}_{2} \mathrm{Cl}_{2}, 4 \AA \mathrm{MS}, 6 \mathrm{~d}, \mathrm{rt}, \mathbf{2 0}, 51 \%$; 21, 42\%; (ii) TBAF, THF, $5{ }^{\circ} \mathrm{C}, 1 \mathrm{~h}$; (iii) pyridine, THF, HF-pyridine complex, $43{ }^{\circ} \mathrm{C}, 17 \mathrm{~h}$, then aq. $\mathrm{NaHCO}_{3}, \mathbf{2 2}, 56 \% ; \mathbf{2 3}, 62 \%$; over two steps. 


\subsubsection{Biological activity of TDMs}

Heterogeneous mixtures of TDMs isolated from Mycobacterium have been tested for their biological activities and have been found to possess a number of biological properties including anti-tumour activity, ${ }^{52}$ adjuvant activity, ${ }^{53}$ anti-bacterial activity, ${ }^{39}$ and the ability to induce granuloma formation, ${ }^{54}$ as well as angiogenesis. ${ }^{55}$ It is only very recently that homogenous trehalose mono- and dimycolates have been tested for biological activity. Synthetic $\alpha$-TDM 22 was found to activate mouse RAW 264.7 M $\varphi$ s to produce TNF (tumor necrosis factor alpha)- $\alpha$ and MCP (monocyte chemoattractant protein)-1 in about three-fold excess compared to a commercial sample of TDM. ${ }^{46}$ Synthetic TMM 23 on the other hand was found to activate M $\varphi$ s at a lesser extent, and the production of TNF- $\alpha$ and MCP observed was half of that for commercial TDM. This finding is particularly interesting as it illustrates how the specific structure of the trehalose glycolipid can influence biological activity.

\subsection{The TDM Receptor: Macrophage Inducible C-type Lectin (Mincle)}

It is widely known that bacteria trigger $\mathrm{M} \varphi$ activation through recognition by the innate immune system ${ }^{56}$ and that $\mathrm{M} \varphi \mathrm{s}$ have pattern recognition receptors (PRRs) for microbial ligands. ${ }^{57}$ These ligands contain pathogen associated molecular patterns (PAMPs), which can be recognised by PRRs. Some PRRs include Toll-like receptors (TLR), Nucleotide binding oligomerisation domain (NOD)-like receptors, and C-type lectins (such as Dectin-1, Dectin-2, and Mincle) ${ }^{57,58}$ These ligand-receptor interactions decode pathogen information by triggering distinct signalling pathways to differentially activate APCs and direct a response which is specific for the invading bactieria. ${ }^{59}$ 


\subsubsection{Binding Mincle and Intracellular signalling}

In 2009, the receptor for TDM was identified as Macrophage Inducible C-type Lectin (Mincle). Mincle (also called CLEC4E) is a C-type lectin with an extracellular $\mathrm{Ca}^{2+}$ dependent carbohydrate domain that contains a mannose binding motif. ${ }^{60}$ Mincle is expressed at low levels on several cell types including monocytes, M $\varphi$ s, neutrophils, myeloid dendritic cells (DCs) and some subsets of B cells, ${ }^{61}$ but is up-regulated in response to a number of signals, including the activation of TLRs, or through an auto amplification loop initiated by Mincle-ligands, ${ }^{62}$ such as the trehalose glycolipids themselves. $^{63}$

Lang and co-workers used various BMM knockout models to determine that C-type lectins, rather than TLRs, recognise the glycolipids TDM and TDB. ${ }^{59}$ BMMs were tested for their ability to respond to TDM and TDB, with activation of BMMs being measured by nitric oxide (NO) and cytokine production. TLRs signal via the adaptor protein MyD88 to induce T helper (Th) 1 directing cytokines, although certain C-type lectin receptors use the kinase Syk to direct Th-17 differentiation. ${ }^{59}$ To determine the pathway required for TDM and TDB mediated cell activation, TDM and TDB stimulated MyD88 $8^{-/-}$and $\mathrm{Syk}^{-/-}$BMMs were compared to stimulated wild-type BMMs. Although $\mathrm{MyD}^{-/ /-}$BMMs retained their normal response, $\mathrm{Syk}^{-/-}$BMMs elicited no response. In a similar manner, the myeloid cell-specific adaptor protein, Card9, was required for the TDM stimulated BMM response, and also the downstream proteins Bcl10 and Malt1. The $\beta$-glucan receptor Dectin-1, which had previously been linked to antigen presenting cell responses to whole mycobacteria, was also excluded as the TDM receptor using Dectin-1 $1^{-/}$BMMs. As a large number of myeloid cell receptors which activate Syk are associated with adaptor proteins Dap12 or $\mathrm{FcR} \gamma$, the requirement for these proteins was tested. FcR $\gamma$, but not Dap12, was found to be essential in linking TDM and TDB recognition to M $\varphi$ activation via the Syk-Card9 signalling pathway. ${ }^{59}$ In a later study, $\mathrm{Mincle}^{-/-}$mice were used to establish that this $\mathrm{FcR} \gamma$-associated receptor is crucial for TDM induced M $\varphi$ activation. ${ }^{11}$ 


\subsubsection{Induction of the Th-1/Th-17 immune response by Mincle}

The overall effect of TDM or TDB binding and activation of Mincle is the induction of a Th-1 and Th-17 immune response (Figure 2). ${ }^{11}$ Stimulation of Mincle through TDM activates this signaling pathway to induce the expression of many different proinflammatory cytokines (e.g. IL-1 $\beta$, IL-6, TNF- $\alpha$ and IFN- $\gamma$ ), chemokines (e.g. MCP-1 and MIP-1 $\alpha$ ) and the release of small molecule cytotoxic mediators, such as iNOS. ${ }^{59,64}$ Importantly, the release of IL-12 by marcophages enhances Th-1 differentiation, while the production of IL-6, TGF- $\beta$, IL-23 and IL-1 $\beta$ favours the development of a Th-17 response. This Th1/Th17 response along with the up-regulation of co-stimulatory molecules (CD80, CD86) and Major histocompatibility complex (MHC)-II induce further expression of Mincle on APCs and the activation of the early adaptive immune response, which is required for the development of protective $\mathrm{T}$ cell immunity. Indeed, the capability of Freund's complete adjuvant and the CAF01 liposomes to induce Th1 and Th17 immunity in mice has been attributed to the ability of trehalose glycolipids to activate the FcR $\gamma$-Syk-Card9 pathway. ${ }^{59,65}$

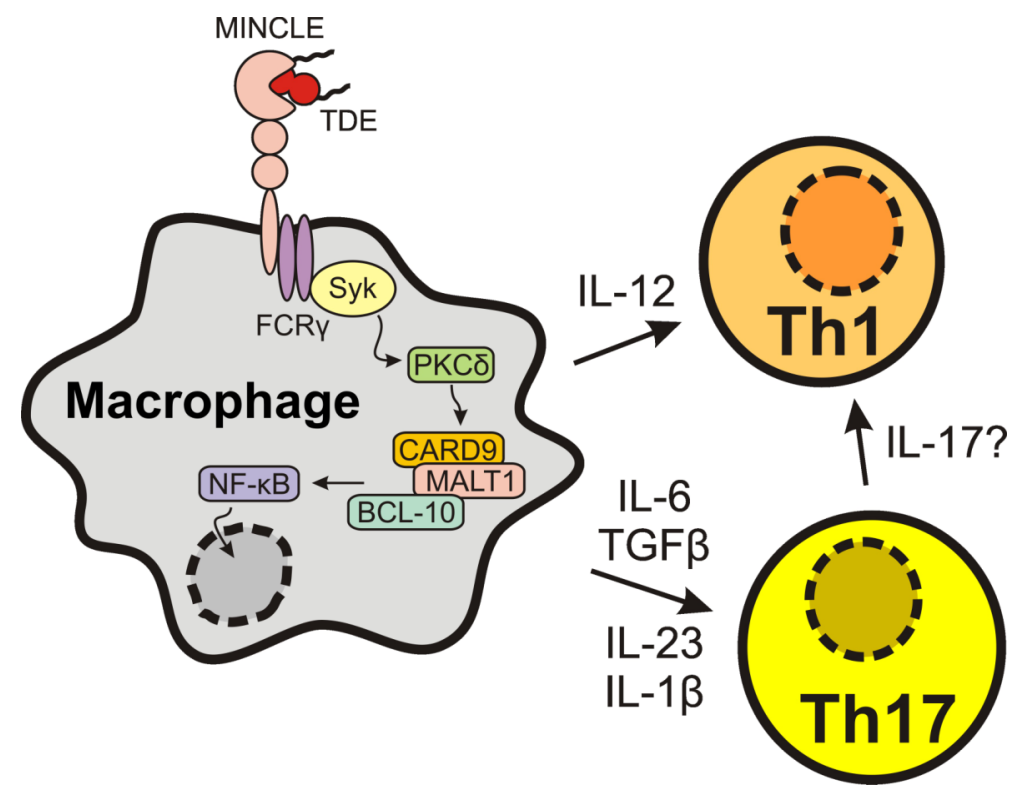

Figure 2. The Th-1/Th-17 immune response by Mincle 


\subsubsection{Ligands for Mincle}

At present, there is much interest in better defining the ligands for Mincle. Mincle has been shown to bind to mannose containing glycolipids from C. albicans and Malassezia species with affinity for $\alpha$-mannose geometry, ${ }^{66,67}$ however, it does not recognise $\alpha-1,2-$ oligomannose-containing glycolipids from the $M$. $t b$ cell wall or any other cell wall components apart from TDM and its derivatives. ${ }^{11}$ Studies by Ishikawa et al. in $2009^{58}$ established that the 1,1- $\alpha$-linked glucose disaccharide (trehalose) motif is essential for the recognition of mycobacteria. As TDB is also capable of activating Mincle expressing cells, ${ }^{11}$ yet neither trehalose alone or the corresponding lipid are able to activate, this suggests that both the sugar head group and the lipid are required for the activation of Mincle. ${ }^{68}$

\subsubsection{Crystal Structure of Mincle}

Very recently, the first crystal structures of Mincle were solved by two independent research groups. ${ }^{69,70}$ Both groups solved the structure with citric acid bound to the carbohydrate recognition domain (CRD), while Feinberg et al. also solved the crystal structure with trehalose bound.

Feinberg et al. used X-ray crystallography, mutagenesis, and competition studies to reveal an extended ligand-binding site in Mincle. ${ }^{69}$ This binding site was found to interact with both the sugar head group and acyl portions of the glycolipid. Characterisation of the Mincle CRD was achieved by comparison of mouse, human and cow Mincle, which revealed that all species had close sequence similarity throughout the polypeptides, including in the C-terminal CRD. Crystals of expressed CRD were then analysed by X-ray crystallography and solved by molecular modelling to reveal that the Mincle polypeptide assumes an overall fold similar to other C-type CRDs. When generated in the presence of citric acid, it is also interesting to note the 
dissimilarity between Mincle and other C-type lectins at the $\mathrm{Ca}^{2+}$ binding site. In the conserved $\mathrm{Ca}^{2+}$ binding site that serves as the primary sugar-binding region, there are normally five amino acid side chains that form the divalent cation-binding site, yet Mincle only has three chains, which coordinate to $\mathrm{Ca}^{2+}$. Generation of the Mincle CRD crystals in the presence of trehalose, however, resulted in a significant change in the organisation of the crystal so that the trehalose complex has five side chain ligands for the conserved $\mathrm{Ca}^{2+}$ ion. This change in conformation and lattice packing was attributed to the presence of saturating trehalose as both crystal structures were obtained under the same conditions. Trehalose-bound Mincle (Figure 3) also assumes a conformation that is observed in crystal structures of unrelated proteins, for example, trehalose/maltose binding protein from Thermococcus litoralis, M. tb antigen 85B, and the C-terminal lobe of bovine lactoferrin. ${ }^{69}$ Here, the two glucose residues are arranged in such a way that one glucose residue brings the second glucose residue into contact with a greater surface area of the protein to form a secondary binding site. In this secondary binding site, the glucose 2-OH forms part of a hydrogen-bonding network that accepts a hydrogen bond from Arg182 and donates to Glu135 to ultimately bridge these two side chains. Arg182 is also important because it is packed against $\mathrm{C} 3$ of the glucose residue in the secondary binding site. The secondary binding site observed in the crystal structure thus suggests that this site may function as part of the binding mechanism for TDM. 


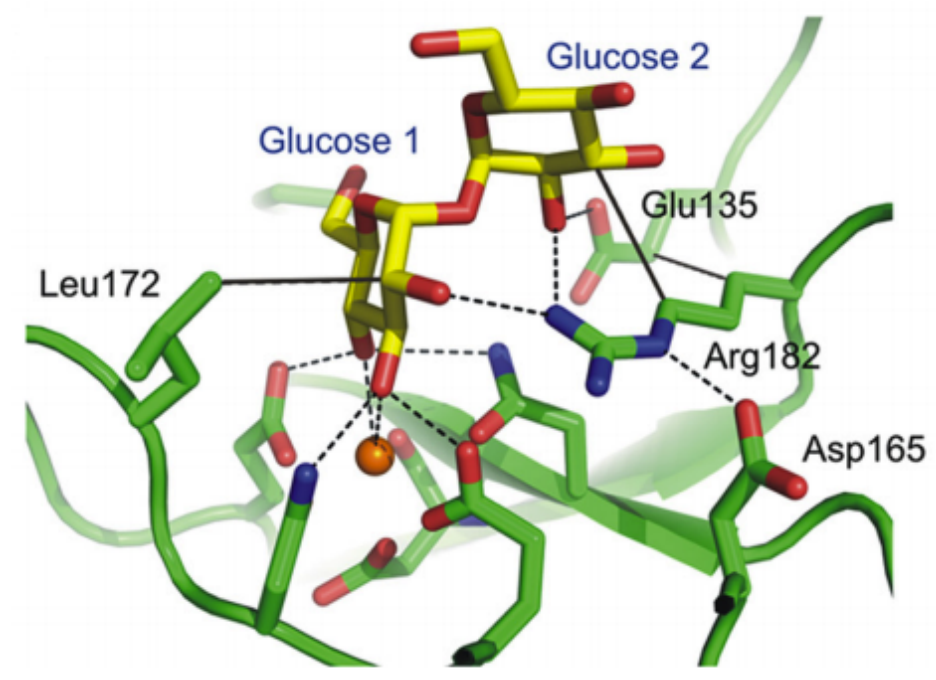

Figure 3. Mincle-trehalose disaccharide interactions at both the primary and secondary binding sites. This research was originally published in The Journal of Biological Chemistry. Hadar Feinberg et al., Mechanism for Recognition of an Unusual Mycobacterial Glycolipid by the Macrophage Receptor Mincle., J. Biol. Chem., 2013; 288: 28457-28465. (C) the American Society for Biochemistry and Molecular Biology. With permission.

To explore the effect of the lipid on TDM binding to Mincle, a series of trehalose esters were prepared using enzymatic syntheses and binding studies revealed that acylation of the two 6-OH groups are tolerated. ${ }^{69}$ This confirms that these groups are positioned freely on the surface of the CRD. Moreover, acylation with 3- or 4- carbon chains results in significantly enhanced affinities for Mincle as observed by the $\mathrm{K}_{1}$ (trehalose) $/ \mathrm{K}_{1}$ (acyltrehalose) values. It is also important to note that the surface of the Mincle CRD near the primary sugar binding site contains a hydrophobic channel which is positioned directly adjacent to the 6-OH group of the primary glucose residue. Modelling studies have suggested that this hydrophobic channel would accommodate at least 6 carbon atoms. Furthermore, as the 6-OH group of the secondary glucose residue is positioned away from the CRD's surface, one of the two lipids on TDM was thought to be less important for Mincle-binding. 
In the second Mincle crystallographic study, Furukawa et al. also report on the crystal structure of the Mincle-citric acid complex and illustrate that Mincle, unlike other Ctype lectins, possesses a unique shallow hydrophobic region located adjacent to the sugar binding sites. ${ }^{70}$ Moreover, Furukawa et al. investigated the effect of the acyl chain on Mincle binding and using surface plasmon resonance (SPR) and a series of trehalose glycolipids with a single acyl chain of different carbon lengths (C8, C10, C12), illustrated that the trehalose with a single $\mathrm{C} 8$ acyl chain bound to Mincle with much lower affinity than those of $\mathrm{C} 10$ and $\mathrm{C} 12$. This highlights the ability of the hydrophobic region to accommodate an acyl chain of at least ten carbon atoms. ${ }^{70}$

\subsection{Liposomes}

Although TDMs and TDEs have many interesting properties when administered alone, their incorporation into liposomes has also found many interesting applications. TDM/TDE liposomes have been used as vaccine adjuvants, ${ }^{52,71-74}$ and to enhance the anti-cancer effect of the glycolipid component. It is important to note, however, that the use of TDM/TDE liposomes as drug delivery vehicles (i.e. to encapsulate drugs) has not been previously explored. Before trehalose glycolipid liposomes are discussed in more detail, a short review on liposome synthesis and properties will first be given.

\subsubsection{Overview}

Liposomes were first prepared by Watkins and co-workers in $1965 .^{75}$ Liposomes resemble cell membranes in structure and composition and are typically made from phospholipids, which are considered to be non-toxic and non-immunogenic. Liposomes consist of one or more concentric lipid bilayers that enclose an internal aqueous volume $^{76}$ and are classified into three categories on the basis of their size and lamellarity 
(number of bilayers): small unilamellar vesicles (SUVs) are between 0.02-0.05 $\mu \mathrm{m}$, large unilamellar vesicles (LUVs) are greater than $0.6 \mu \mathrm{m}$, and multilamellar vesicles (MLVs) range from 0.1-6 $\mu \mathrm{m} .^{77,78}$

The ability to encapsulate or bind a variety of drugs into or onto the membrane of liposomes makes them attractive candidates as drug delivery vehicles and their clinical applications include use as chemotherapies for cancer and fungal infections, and vehicles for vaccination and gene therapy. ${ }^{76}$ The versatile nature of liposomes means that many different components can be added or removed and their properties can thus be tailored to the respective application. For example, drugs can be incorporated in liposomes in the hydrophobic hydrocarbon core, on the polar surface, or in the internal aqueous space. $^{76}$

\subsubsection{Synthesis of Liposomes}

The method used to prepare liposomes is important because it determines the type of liposome formed. The general preparation of liposomes involves four stages - removal of the organic solvent to form the lipid, dispersion of the lipid into aqueous media, purification of the resultant liposome, and then characterisation. Generally, MLV are prepared using the method reported by Watkins and co-workers, ${ }^{75}$ whereby a lipid mixture in organic solvents was allowed to deposit upon the walls of a round bottom flask by rotary evaporation under reduced pressure. The thin lipid film is then dispersed in aqueous buffer at a temperature above the transition temperature $\left(\mathrm{T}_{c}\right)$ of the lipid to disperse large lipid aggregates. On the other hand, SUVs are prepared by injecting an aqueous suspension of lipids under high pressure through a "French press" and the liposomes can be subsequently fractionated by size, by centrifugation, or molecular sieve chromatography. ${ }^{79}$ LUVs are prepared via ether injection, in which an ether solution of lipid is injected into an aqueous medium. ${ }^{79}$ Several modifications have been 
made to the general methods described above so as to tailor the exact properties of the liposomes.

\subsubsection{Liposomes in Immunology}

Liposomes generally used in immunological studies are multilamellar in structure because of the ease of encapsulation of proteins and the variety of lipid compositions that can be used. ${ }^{80}$ Here, a long hydration and gentle shaking rather than a faster and more vigorous preparation can achieve a higher percentage of encapsulation per mole of lipid. ${ }^{80}$ In addition, egg lecithin or phosphatidylcholine (PC) are often considered to be important materials of liposomes used for adjuvant activity because both substances are biodegradable and non-toxic when administered as liposomes. ${ }^{80,81}$ More importantly, the advantage of phosphatidylcholine liposomes as adjuvants is that PC is a very poor antigen, as compared to other lipids such as phosphatidyl inositol, phosphatidyl glycerol, and phosphatidic acid. ${ }^{80}$

There are two major interactions between liposomes and cells that must be considered in liposome applications for drug delivery. ${ }^{82}$ The most important interaction is lipid exchange and is a long-range interaction that involves the exchange of liposomal lipids for the lipids in the cell membrane of various cells. The second major interaction is adsorption. Adsorption occurs when the attractive forces (including electrostatic, van der Waals, hydrophobic insertions and hydrogen bonding) exceed the repulsive forces (electrostatic, steric, hydration). ${ }^{82}$ These interactions depend on the surface characteristics of the liposomes, such as charge and polarity. 


\subsubsection{Liposomes in Drug Delivery}

"Conventional" liposomes are liposomes composed of neutral and/or negatively charged lipids and cholesterol. Some of these conventional liposome formulations have reached the market, or are now entering clinical trials. For example, Ambisome ${ }^{\circledR}$ is a liposome formulation that encapsulates the antifungal amphotericin $B$ drug, Myocet ${ }^{\circledR}$ encapsulates the anticancer agent doxorubin, and Daunoxome ${ }^{\circledR}$ has the drug daunorubicin incorporated into the liposome. ${ }^{83}$ The advantage of incorporating drugs into liposomes includes the ability to target specific cells, tissues or organs and enable the delivery of higher drug doses. ${ }^{84}$ Additionally, as the drugs are encapsulated in liposomes, they are expected to be transported without rapid degradation and with minimum side effects to the recipients. ${ }^{84}$

Another type of liposome is the long-circulating liposome, which is based on the concept that steric stabilisation of the liposome increases its longevity in circulation. Here, a hydrophilic polymer or glycolipid, such as polyethylene glycol (PEG), which possess a flexible chain, can occupy the space immediately adjacent to the liposome surface ("periliposomal layer") to exclude other macromolecules from this space. ${ }^{83}$ As a consequence, access and binding of blood plasma opsonins to the liposomal surface is hindered, thereby inhibiting interactions between $\mathrm{M} \varphi \mathrm{s}$ and the liposome, ${ }^{83}$ which is the main means by which liposomes are removed from circulation. Polyethylene glycols are also known to increase drug stability and solubility and lower toxicity, and when placed on the liposomal surface they prevent vesicle aggregation, thus improving the stability of formulations. ${ }^{83}$ PEG incorporation is achieved by anchoring the polymer in the liposomal membrane using a cross-linked lipid [i.e. PEGdistearoylphosphatidylethanolamine (DSPE)]. ${ }^{83}$ The successful application of Stealth liposomes is highlighted by the FDA approval of DOXIL® (Stealth liposomal doxorubicin $\mathrm{HCl}$ ) for the treatment of Kaposi's sarcoma in $1995 .{ }^{85}$ 


\subsubsection{TDM/TDE Liposomes}

There have been several studies where TDM or TDB have been incorporated into liposomes. In early work, Nolibe et al. administered TDM-liposomes suspended in saline to Wister AG rats by the intratracheal (i.t.) route and monitored the activation of alveolar M $\varphi s$ as well as the activation of NK (natural killer) cells of the lung. ${ }^{52}$ Their results showed that TDM-liposomes administered via i.t. induced a transient inflammatory effect, such that the activated M $\varphi$ s had cytostatic activity against P77 fibrohistiocytoma three days after administration. However, the NK activity of lymphocytes of the lung did not increase, which suggests that M $\varphi s$ may be the main effector cells responding to TDM. In addition, Nolibe et al. found that repeated i.t. TDM adminstration protected rats against the development of colonies arising from intravenously (i.v.) injected tumor cells. More recently in 2013, Carlétti et al. investigated the immune protection of an alanine proline antigen (Apa)-TDM-DNA vaccine against intratracheal $M$. tuberculosis challenge. ${ }^{71}$ Here, BALB/c mice were primed with BCG and a single dose of plasmid carrying Apa and TDM adjuvant coencapsulated in liposomes for a prime-boost strategy. Evaluation at 30 and 70 days post challenge showed that this prime-boost strategy resulted in a significant reduction in the bacterial load in the lungs and thus, the strategy holds much promise for the prevention of tuberculosis.

The structurally related TDB has also been used in liposomal formulations. Kamath et al. and Gram et al. both investigated the liposome-based adjuvant CAF01 (also referred to as DDA-TDB or Lipovac) in mouse models. ${ }^{72,73}$ The CAF01 adjuvant is based on cationic liposomes formed by the quarternary ammonium lipid $N, N^{\prime}$-dimethyl- $N, N^{\prime}-$ dioctadecylammonium (DDA) and TDB, and when combined with the TB antigen Ag85B-ESAT-6, was used in Phase I clinical trials as a TB vaccine. ${ }^{74}$ Kamath et al. found that following adult or neonatal murine immunisation with Ag85B-ESAT-6 (5 $\mu \mathrm{g})$ formulated in CAF01, the post-challenge bacterial growth of $M$. bovis $\mathrm{BCG}$ was reduced when compared to that observed using Ag85B-ESAT-6 formulated in aluminium 
hydroxide (the control). ${ }^{72}$ Gram et al. also found that the CAF01 adjuvant induced a CD8+ T cell immune response against HIV-1 proteins in HLA-A*0201 transgenic mice that is comparable to that of incomplete Freund's adjuvant. ${ }^{73}$ As CAF01 liposomes are a heterogeneous population with a mean vesicle size of $500 \mathrm{~nm}$, Henriksen-Lacey et al. investigated whether various sized CAF01 liposomes in combination with Ag-85BESAT-6 exhibited altered pharmacokinetics, cellular uptake and activation in vitro. ${ }^{74}$ Their study showed that while there were no differences in the vaccine draining profile from the injection site, there were, however, significant changes in the movement of liposomes from the lymph node. Different size CAF01 liposomes showed a sizedependent movement, where at both $6 \mathrm{~h}$ and $24 \mathrm{~h}$ post-injection, significantly higher levels of large $(\sim 1.5 \mu \mathrm{m})$ compared to small $(\sim 200 \mathrm{~nm})$ DDA:TDB liposomes were detected in the popliteal lymph node. Milicic et al. further explored the effect of size of the DAA:TDB liposomes and determined that cationic DDA:TDB liposomes of the same chemical composition but different size and lamellarity differed in their ability to induce cellular immune responses. ${ }^{86}$ Small unilamellar liposomes of approximately 600 $\mathrm{nm}$ in diameter when combined with Ovalbumin (OVA) protein were able to induce a higher cellular adaptive immune response than multilamellar vesicles with a two-fold larger diameter. This finding was unexpected as a smaller amount of OVA protein is incorporated in SUVs compared to MLVs (85\% versus $95 \%$ of total dose of OVA protein). Thus, it is possible that a difference in the endocytic pathways involved in the internalisation of the two types of liposomes affects antigen presentation, and hence, the ensuing immune response. 


\subsection{Macrophages in disease}

M $\varphi s$ are innate immune cells and are one of the most versatile cells in the body. They play a critical role in innate immunity, inflammation and repair, ${ }^{87,88}$ and are the first line of defence against invading pathogens. ${ }^{89}$ To distinguish between infectious agents and self, a number of $\mathrm{M} \varphi$ phagocytic receptors have evolved, including the mannose receptor, which is involved in the recognition of PAMPs on pathogens. ${ }^{90} \mathrm{M} \varphi$ are derived from hematopoietic cells that develop into monocytes in blood and which, upon entering tissues, differentiate into $\mathrm{M} \varphi \mathrm{s} .{ }^{89} \mathrm{M} \varphi$ are found in essentially all tissue, including the lungs, liver, brain, bone and skin, and depending on the tissue environment, are capable of different physiological functions. ${ }^{91}$ As discussed previously, $\mathrm{M} \varphi$ express Mincle and thus can interact with TDM and TDE.

$\mathrm{M} \varphi$ have the distinctive ability to be able to polarise to different phenotypes that express unique cell surface molecules and secrete discrete sets of cytokines and chemokines. The classically activated, or 'M1' phenotype, supports the pro-inflammatory Th-1 response that is driven by cytokines such as IL-12 and IL-23, while the alternative 'M2' phenotype is supportive of anti-inflammatory processes driven by IL-10. ${ }^{91}$ The ability of $\mathrm{M} \varphi$ to change phenotype is known as $\mathrm{M} \varphi$ plasticity, which, in some instances, can lead to or enhance disease. For example, tumour-associated macrophages (TAMs) are found in large numbers in solid tumours, ${ }^{92,93}$ and exhibit the alternatively activated ' $\mathrm{M} 2$ ' phenotype associated with immunosuppression, promotion of tumour angiogenesis and metastasis. As M $\varphi$ s are thought to play a role in the pathogenesis of diseases, the specific targeting and destruction of M $\varphi$ s may be a strategy that could find application in the treatment of cancer, ${ }^{94}$ and tuberculosis, whereby in the latter case, M.tb is known to reside inside the $\mathrm{M} \varphi$ and not be destroyed. ${ }^{95}$

Early methods of depletion of M $\varphi s$ were based on the administration of silica, carrageenan or by various other treatments, ${ }^{96}$ however, depletion was often incomplete and unwanted side effects on other non-phagocytic cells were a disadvantage. Thus, a 
more sophisticated approach was developed based on a liposome medicated intracellular delivery of the $\mathrm{M} \varphi$ depleting drug bisphosphonate clodronate. This approach has proven to be effective in several tumour models, ${ }^{97,98}$ such as the murine F9 teratocarcinoma and the human A673 rhabdomyosarcoma mouse tumour models, ${ }^{99}$ and also in clinical trials for patients with breast cancer. ${ }^{100}$ As only a small fraction of drugs that are injected in soluble form into the body reach $\mathrm{M} \varphi \mathrm{s},{ }^{101}$ there has been much interest in developing carrier systems, such as liposomes and microspheres, that will allow for the specific targeting of $\mathrm{M} \varphi$. In this manner, liposomes are used as a Trojan horse to deliver

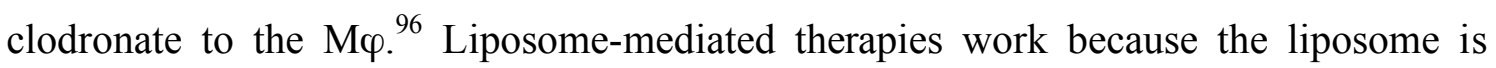
preferentially engulfed by the phagocytic $M \varphi$, however, for the strategy to be effective, M $\varphi s$ must be activated in such a way that phagosome-lysosome fusion is induced and a high intracellular threshold of clodronate is reached. ${ }^{102}$ Thus, increasing the degree of $\mathrm{M} \varphi$ activation remains an important obstacle in the development of better $\mathrm{M} \varphi$ depletion therapies. It is envisioned that this can be achieved by incorporating trehalose glycolipids into liposomes, which is an area of TG-liposome research that hasn't been explored. 


\subsection{Research aims}

The objective of this MSc thesis was to:

a. Explore the immunomodulatory potential of trehalose glycolipids (TGs), in particular, whether TMEs will bind and activate M $\varphi s$ via Mincle.

b. Explore the potential of liposomes containing TGs, specifically TDEs and TME, with the future objective of developing improved drug delivery systems.

The aim to improve the synthesis of the TMEs to further explore the Mincle binding site and investigate the structural requirements for activation of $\mathrm{M} \varphi$ s will be discussed in Chapter Two. To this end, the synthesis of TMEs was undertaken and the ability of these monoesters to activate $\mathrm{M} \varphi$ explored, and compared to the biological properties of their diester counterparts.

In Chapter Three, the effects of non-functionalised (or empty) liposomes, as well as TG containing liposomes on $\mathrm{M} \varphi$ activation were determined (by way of establishing NO production by $\mathrm{M} \varphi$ ). TG-liposomes synthesised contained the most active TG. These experiments thus represent the first steps to determining whether the addition of TG into liposomes can lead to better $\mathrm{M} \varphi$ depletion strategies. 


\section{CHAPTER 2:}

\section{SYNTHESIS AND BIOLOGICAL EVALUATION OF C26-TME}

\subsection{Overview}

There is much interest in the synthesis of trehalose diesters (TDEs) and their derivatives due to their interesting biological properties. Previous work performed within our laboratory by Khan et al. led to the first report of how TDE lipid length affects the innate immune response of $\mathrm{M} \varphi \mathrm{s} .{ }^{28}$ Results from this work indicated that longer lipids $\left(\geq \mathrm{C}_{18}\right)$ are required for the activation of $\mathrm{M} \varphi \mathrm{s}$, whereas TDEs with shorter chain lipids are inactive. ${ }^{28}$ This observation is significant as it provides an insight into why M.tb, which expresses long chain TDEs, has the ability to trigger an innate immune response. The synthetic route developed by Khan et al. for the formation of TDEs also led to the formation of trehalose monoesters (TMEs) as a side product.

In this chapter, I describe the synthesis of the C26 TDE, as well as exploration of methodology to allow for the efficient synthesis of TMEs. The TMEs were then tested to determine if they activate $M \varphi$, and if $M \varphi$ activation is dependent on Mincle. These results will provide valuable structure-activity relationship data.

\subsection{Synthesis of TDE}

The synthetic strategy developed by Khan et al. was used to prepare the required C26 TDE for incorporation into liposomes. ${ }^{28}$ To this end, trehalose 24 was treated with $N, O$ - 
bis(trimethylsilyl)acetamide (BSA) and catalytic tetra-butylammonium fluoride (TBAF), then with $\mathrm{K}_{2} \mathrm{CO}_{3}$ to generate diol 19 in a one-pot procedure whereby 24 was first per-silylated, and then the more labile primary silyl ethers removed during work up by the addition of $\mathrm{K}_{2} \mathrm{CO}_{3}$ (Scheme 4 ).

The TMS-protected derivative $\mathbf{1 9}$ was then coupled to hexacosanoic acid under the mediation of 1-ethyl-3-(3-dimethylaminopropyl)carbodiimide (EDCI) and 4dimethylaminopyridine (DMAP) in $\mathrm{CH}_{2} \mathrm{Cl}_{2}$ to give diester 25 in $60 \%$ yield. The TMS groups were then readily removed following subjection of the diester $\mathbf{2 5}$ to Dowex- $\mathrm{H}^{+}$ resin to yield the target C26 TDE 26 in 70\% yield.

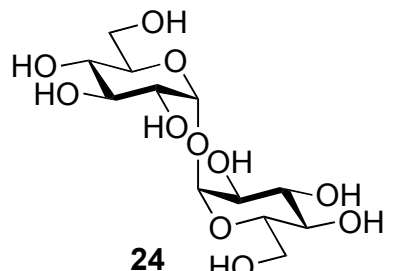

24
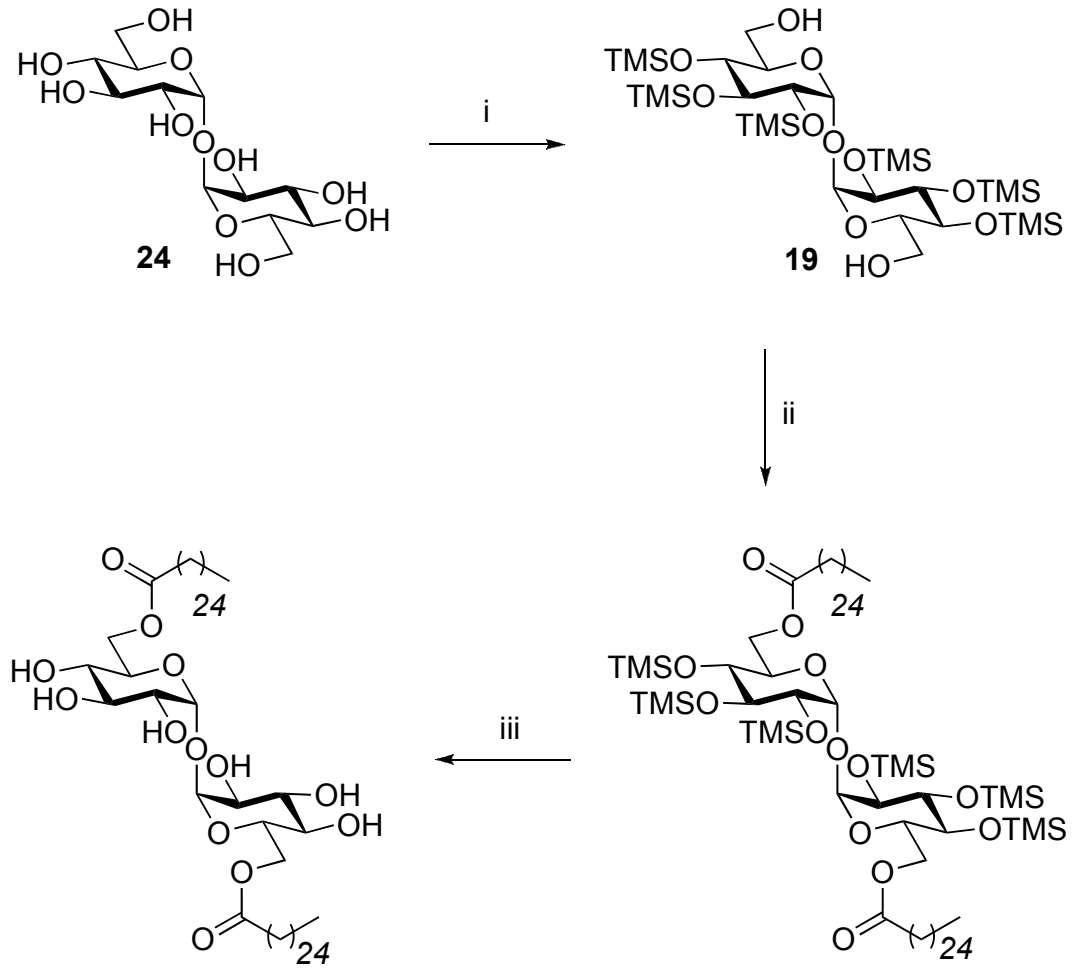

26

$$
\downarrow
$$

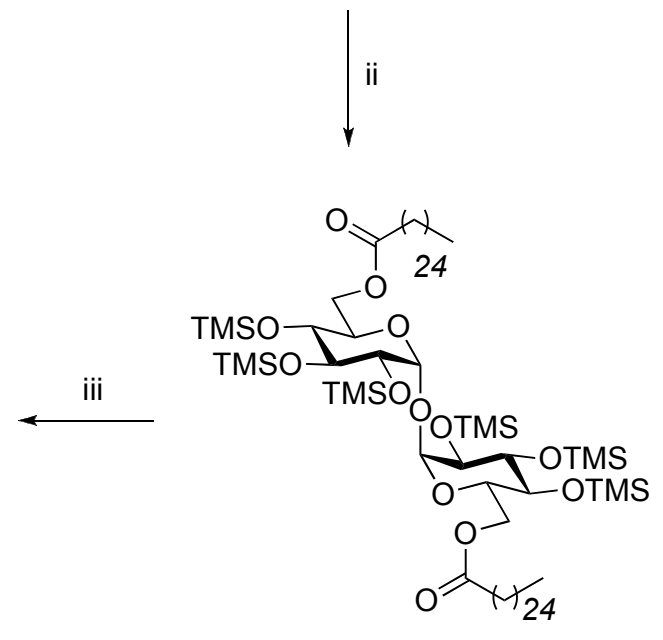

25

Scheme 4. Synthesis of TDE 26 (C26-diester); Reagents and conditions: i) BSA, TBAF (cat.), DMF, RT, 30 min, then propan-2-ol, $0{ }^{\circ} \mathrm{C}, \mathrm{K}_{2} \mathrm{CO}_{3}, 2 \mathrm{~h}$, $85 \%$; ii) hexacosanoic acid, EDCI, DMAP, 60\%; iii) Dowex- $\mathrm{H}^{+}$, $\mathrm{CH}_{2} \mathrm{Cl}_{2} / \mathrm{MeOH}$ (1:1), RT, 30 min, 70\%. 
The mechanism for the BSA-mediated silylation reaction commences with attack by the fluoride anion in tetra-butylammonium fluoride (TBAF) on to the silyl atom in $\mathrm{N}, \mathrm{O}$ bis(trimethylsilyl)acetamide (BSA) (I) to generate intermediate II (Scheme 5). Attack on this silyl group is preferred due to the formation of a stable intermediate II, whereby the negative charge on the oxygen is resonance stabilised to form intermediate III. The negatively charged nitrogen atom in III then deprotonates a proton from one of the hydroxyl groups in trehalose to give a negatively charged oxygen atom in $\mathbf{I V}$, which then attacks a silyl group in another molecule of BSA (I) and results in the formation of a TMS ether (V) and regeneration of intermediate II. 

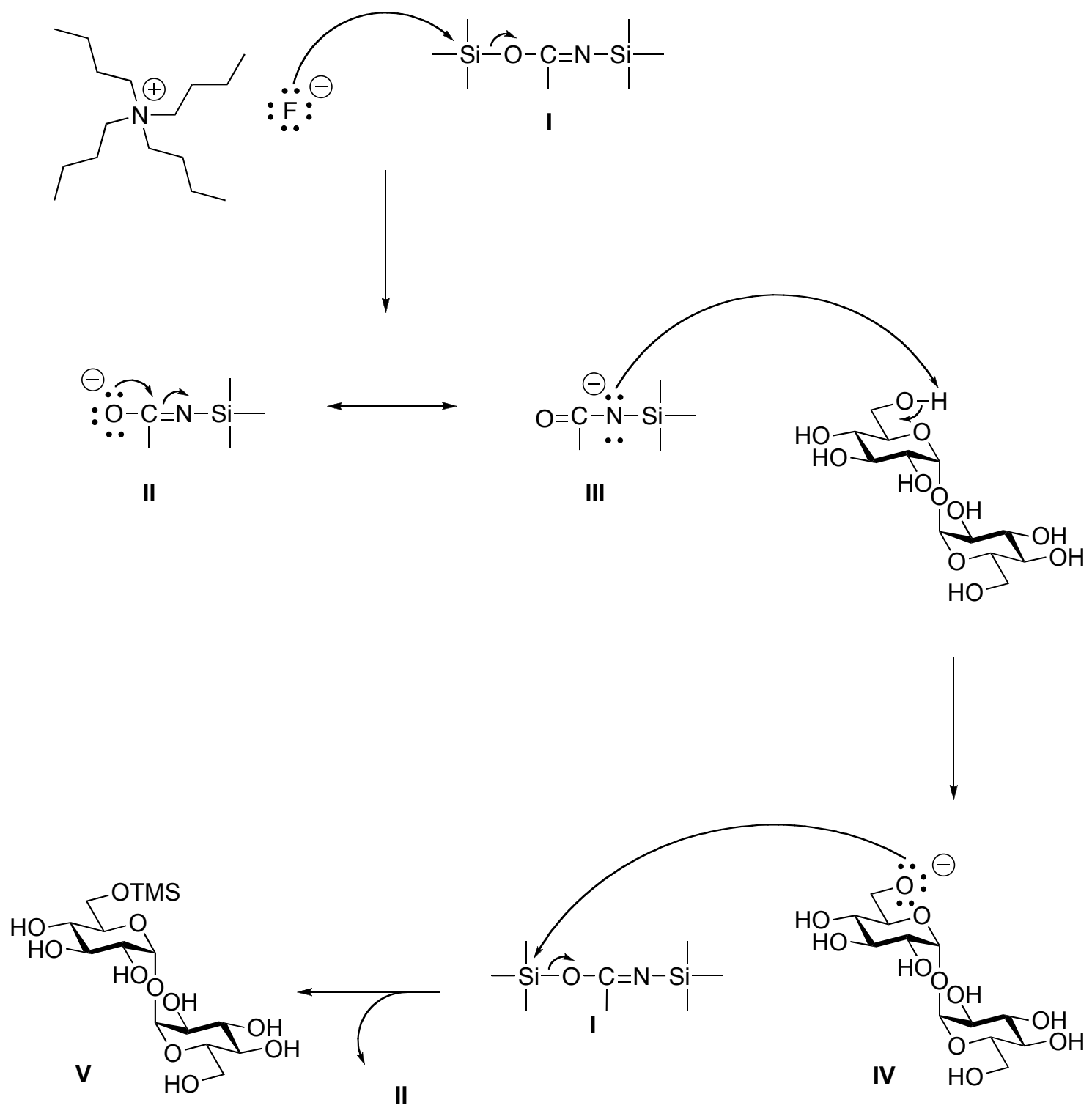

Scheme 5. Reaction mechanism for BSA mediated silylation of trehalose. ${ }^{28}$ 


\subsection{Synthesis of TME}

Although the formation of TDEs can be achieved as described above, the synthesis of very-long chain $\left(\geq \mathrm{C}_{20}\right)$ TMEs is nonetheless challenging as it is difficult to optimise mono- versus di-esterification. The reactivity of long-chain lipids is hindered by their poor solubility in many organic solvents and there are only few published syntheses for the formation of TMEs.

In the literature, several strategies for the synthesis of medium to long-chain TMEs have been documented, ${ }^{103-105}$ and these methods include both enzymatic and non-enzymatic strategies. Csuk et al. reported on a convenient, protecting group free route for the synthesis of 6-O-trehalose monoester derivatives from trehalose. ${ }^{103}$ Their procedure is based on the selective chemoenzymatic monoesterification of trehalose to give TMEs in a synthesis without protecting groups in good yield (Scheme 6). ${ }^{103}$ In a typical reaction, trehalose $\mathbf{2 4}$ is reacted with vinyl oleate $\mathbf{2 7}$ in the presence of Alcalase, a commercially available enzyme from Bacillus licheniformis, for 18 days to give 6-O-mono derivative 29 in $72 \%$ yield. Under these same conditions, reaction of 24 and 13methylmyristoyl ester $\mathbf{2 8}$ allowed for the formation of monoacylated derivative $\mathbf{3 0}$ in $74 \%$ yield.
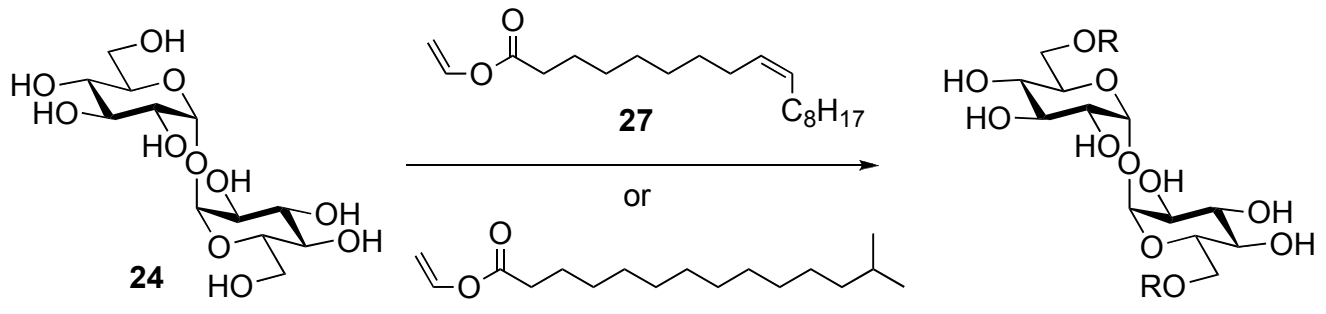

28

Alcalase (B. licheniformis)

$29 \mathrm{R}=$ oleoyl $(72 \%)$

$30 \mathrm{R}=13-$ Me-myristoyl (74\%)

Scheme 6. Chemoenzymatic synthesis of mono-acylated trehalose derivatives 29 and $\mathbf{3 0}$ by Csuk et al. ${ }^{103}$ 
Other groups who have used enzymatic reactions to prepare fatty acid esters of trehalose are Tsuzuki et al. and Woudenberg-van Oosterom et al. ${ }^{104,105}$ Tsuzuki et al. investigated the use of a modified Lipase $\mathrm{P}$ from Pseudomonas sp. as a catalyst for the interesterification between an alcohol and fatty acid. Their results showed that 6-Opalmitoylsucrose, 6-O-palmitoyllactose, 6- $\mathrm{O}$-palmitoylmaltose, and 6-Opalmitoyltrehalose diesters were the dominant products and only minor amounts $(<$ $10 \%$ ) of the monoester were observed. ${ }^{105}$ As the monoester was formed in such small amounts, this methodology is not suitable if TMEs are the target compounds.

Woudenberg-van Oosterom et al. studied the acylation of several disaccharides, including lactose, sucrose and trehalose, among others, using ethyl butanoate and ethyl dodecanoate in the presence of catalyst Candida antarctica lipase in tert-butyl alcohol. ${ }^{104}$ This group observed that the relative reaction rates of the various disaccharides were directly related to their solubility and the ratio of diester to monoester was markedly dependent on the structure of the disaccharide. In general, the first product formed was the monoester, derived from acylation of one of the primary alcohol groups. At greater substrate conversion rates, more diester product was observed, though the ratio of mono:di-ester product was dependent on the disaccharide structure. Interestingly, they noted the reaction of maltose with ethyl dodecanoate afforded only monoester, 6'-monododecanoate at a conversion of $17 \%$ after 24 hours, which increased to $34 \%$ after 48 hours. In contrast, trehalose afforded a $66 \%$ conversion to an equimolar mixture of mono- and diesters in 24 hours and a $86 \%$ conversion after 48 hours, while the amount of monoester did not increase. ${ }^{104}$ On the other hand, lactose reacted at a very slow rate, with $2 \%$ conversion under the same reaction conditions. ${ }^{104}$ Woudenberg-van Oosterom et al. suggested that carbohydrate solubility governs the rate of reaction, and that solubility is determined by the crystal lattice energy and by its solvation energy. As the melting point (MP) is a rough measure for the crystal lattice energy, the varying rates of conversion can be explained by the fact that trehalose has a MP of $98^{\circ} \mathrm{C}$, while lactose has a MP of $204^{\circ} \mathrm{C}$. 
The Friess and Grindley groups have explored non-enzymatic methodology for the synthesis of TMEs. The Friess group describe the design and synthesis of trehalose fatty acid monoesters in four steps to produce high purity products. ${ }^{106}$ Their method begins with the silyl protection of $\alpha, \alpha^{\prime}$-trehalose using the silylating agents trimethylsilyl chloride and hexamethyldisilazane in anhydrous pyridine, followed by selective deprotection at the 6- and 6 '- positions using methanolic potassium carbonate in a manner similar to that previously described for the synthesis of the TDEs. Monoacylation of the symmetrical diol was then achieved using Steglich esterification conditions ${ }^{107}$ with palmitic acid, lauric acid or capric acid. The desired monoesters were obtained in high yields of $72-78 \%$ following removal of the remaining six silyl protecting groups. ${ }^{106}$ In contrast, Grindley's group published a protecting-group-free synthesis of 6-monoesters and 6,6'-diesters of trehalose using a primary-selective acylation procedure. ${ }^{108}$ They showed that reaction of trehalose with one equivalent of a fatty acid in pyridine promoted by one equivalent of the uronium-based coupling agent 2-(1H-benzotriazole-1-yl)-1,1,3,3-tetramethyluronium tetrafluoroborate (TBTU) at room temperature afforded the hexanoic, palmitic or oleic monoester in good yield (65 $69 \%$ ), along with a small amount of diester product. ${ }^{109}$

The synthetic route towards the generation of very-long chain TMEs ( $\geq 22$ carbons), however, is less widely documented and has only been reported a few times for the preparation of trehalose monocorynomycolates (TMCMs) ${ }^{110-112}$ and the synthesis of trehalose monomycolate en route to TDMs (as shown in Scheme 3). ${ }^{46}$ The remarkable differences between long (13 - 21 carbons) and very long-long chain fatty acids is reactivity, which is attributed to differences in solubility and conformation.

Accordingly, I explored the use of TMS-protected trehalose en route to the preparation of very-long chain lipophilic TMEs $\left(\geq \mathrm{C}_{22}\right)$. To this end, the monoester, C26 TME 32, was synthesised using a similar three-step synthetic strategy to that described for the synthesis of the C26 TDE. Here, $\alpha, \alpha^{\prime}$-trehalose (24) was again treated with BSA, catalytic TBAF and $\mathrm{K}_{2} \mathrm{CO}_{3}$ to generate TMS-protected diol 19 (Scheme 7). 
Monoesterification of diol 19 with hexacosanoic (C26) acid to give monoester 31 was then investigated to optimise the reaction conditions. A number of conditions were employed with the objective of increasing the ratio of monoester 31 compared to the undesired C26 diester 25 or starting material 19.

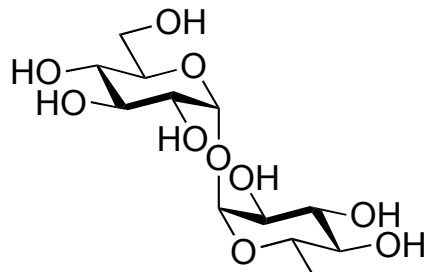

24

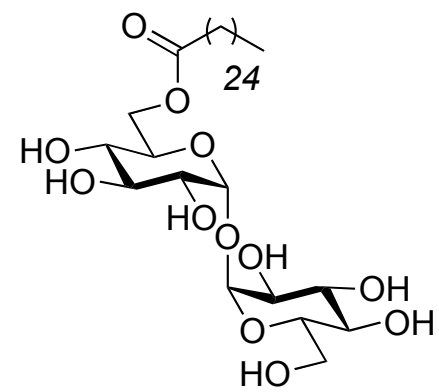

32

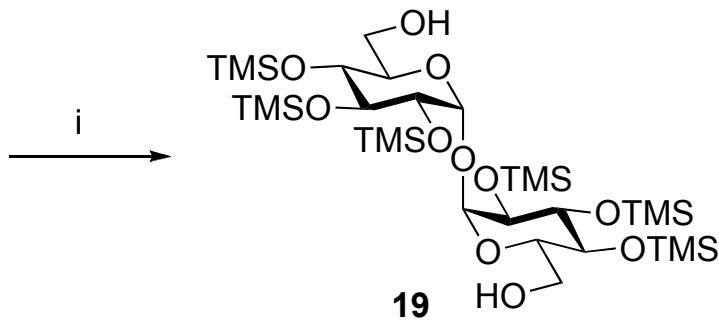

ii

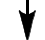

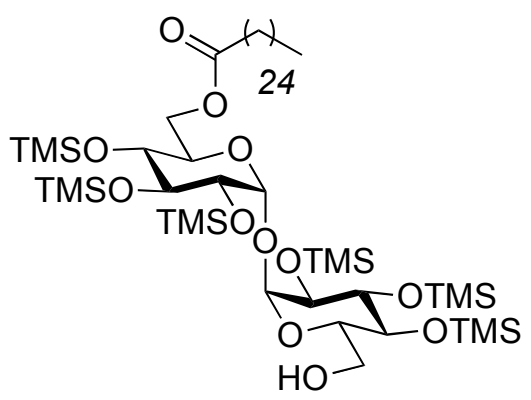

31

Scheme 7. Synthesis of TME 32 (C26-monoester); Reagents and Conditions: i) BSA, TBAF (cat.), DMF, RT, 30 min, then propan-2-ol, $0{ }^{\circ} \mathrm{C}, \mathrm{K}_{2} \mathrm{CO}_{3}, 2$ h, $85 \%$; ii) hexacosanoic acid, EDCI, DMAP; iii) Dowex- $\mathrm{H}^{+}$, $\mathrm{CH}_{2} \mathrm{Cl}_{2} / \mathrm{MeOH}$ (1:1), RT, 30 min, 97\%.

Previously, higher reaction temperatures $\left(70{ }^{\circ} \mathrm{C}\right)$ were used for the synthesis of trehalose diesters, ${ }^{28}$ however, in an attempt to favour formation of monoester $\mathbf{3 1}$ the reaction was first performed at room temperature using different equivalents of acid (entries 1-2, Table 1). In both instances, the reactions were slow and mainly starting material was isolated with monoester 31 being formed in very poor $(<8 \%)$ yield. Here, it should also 
be noted that ${ }^{1} \mathrm{H}$ NMR could not be used to distinguish the ratio of products to starting material due to overlapping signals in the spectra and thus, the C26 TME and C26 TDE needed to be separated by column chromatography and the yield and ratio of the monoester to diester is based on isolated material.

Table 1. Optimisation of synthesis of mono-ester 31.

\begin{tabular}{|c|c|c|c|c|c|c|c|}
\hline \multirow{3}{*}{ Entry } & 19 & $\begin{array}{l}\text { Hexa } \\
\overline{\mathrm{DM}}\end{array}$ & $\begin{array}{l}\text { cosanoic } \\
\text { acid }\end{array}$ & ISO & \multicolumn{2}{|c|}{ 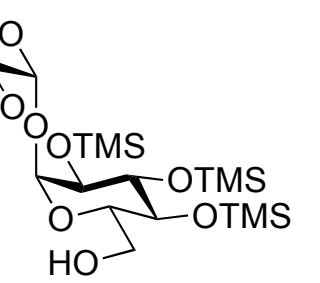 } & 25 \\
\hline & Temp & Time & Acid (equiv.) & DMAP & EDCI & Ratio $^{a}$ & Yield $31(\%)^{\mathrm{a}}$ \\
\hline & & & & & & $31: 25$ & \\
\hline 1 & $\mathrm{RT}$, & & 1.5 & 0.1 & 1.8 & 31 only & $5 \%$ \\
\hline 2 & $\mathrm{RT}$, & & 1.8 & 0.1 & 2.0 & 31 only & $8 \%$ \\
\hline 3 & $70^{\circ} \mathrm{C}$ & $6 \mathrm{~d}$ & 4.0 & 0.1 & 5.0 & $1: 3$ & $20 \%$ \\
\hline 4 & $70^{\circ} \mathrm{C}$ & $4 \mathrm{~d}$ & 1.3 & 0.1 & 1.8 & $33: 20$ & $33 \%$ \\
\hline 5 & $70^{\circ} \mathrm{C}$ & $7 \mathrm{~d}$ & 1.8 & 0.4 & 2.0 & $19: 1$ & $38 \%$ \\
\hline 6 & $70^{\circ} \mathrm{C}$ & $7 \mathrm{~d}$ & 1.8 & 0.2 & 4.0 & $7: 4$ & $35 \%$ \\
\hline 7 & $70^{\circ} \mathrm{C}$ & $7 \mathrm{~d}$ & 1.8 & 4.4 & 4.0 & $9: 3$ & $45 \%$ \\
\hline
\end{tabular}

${ }^{\mathrm{a}}$ Based on isolated yield following purification by silica gel flash column chromatography

As the reactions at room temperature were slow, the reaction was then warmed to $70{ }^{\circ} \mathrm{C}$ to aid with the solubility of the lipid. Here, excess hexacosanoic acid (4.0 equiv.), EDCI (5.0 equiv.) and catalytic DMAP at $70{ }^{\circ} \mathrm{C}$ gave diester 25 preferentially (entry 3), albeit in poor yield. TLC analysis of the reaction progress also illustrated that monoester $\mathbf{3 1}$ 
and diester 25 were formed simultaneously. Next, I set out to determine whether monoester 31 could be formed in preference to diester 25 by varying the equivalents of acid and the reaction time. To this end, 1.3 equivalents of acid with a reaction time of 4 days led to an improvement in the yield of $\mathbf{3 1}$ (entry 4), and adding further acid (1.8 equiv.), DMAP (0.4 equiv.) and increasing the reaction time to one week allowed for the synthesis of $\mathbf{3 1}$ in 38\% yield (entry 5). Subsequent studies, whereby the amounts of DMAP and EDCI were varied (entries 6 and 7) allowed for an optimised 45\% yield of monoester 31 to be obtained. During these later experiments, it was observed that an excess of DMAP, rather than the normal catalytic amount, proved crucial in improving the overall yield of monoester 31 (entry 7). To explain why excess DMAP was required to improve the reaction yield, it is presumed that the use of excess DMAP prevents the formation of $N$-hexacosanoyl urea, ${ }^{113}$ which is a well-known by-product of the Steglich esterification, ${ }^{107}$ particularly when using very long chain carboxylic acids that undergo slow esterification. To explain this mechanistically (Scheme 8), deprotonation of the carboxylic acid by EDCI and subsequent nucleophilic attack of the carboxylate at the carbodiimide occurs, but rather than attack by DMAP to give an activated ester (pathway B), which then reacts with alcohol 19 to give the desired monoester 31, an intramolecular reaction occurs (pathway A). Thus, intermolecular attack from the diimide nitrogen of the activated acid onto the carbonyl of the ester occurs to give the undesired $N$-hexacosanoyl urea side-product. Thus, excess DMAP was added to increase the likelihood of the formation of the DMAP activated ester. ${ }^{107}$ Finally, to complete the synthesis of TME 32, the TMS-groups in $\mathbf{3 1}$ were then deprotected with Dowex- $\mathrm{H}^{+}$ resin in $97 \%$ yield. 


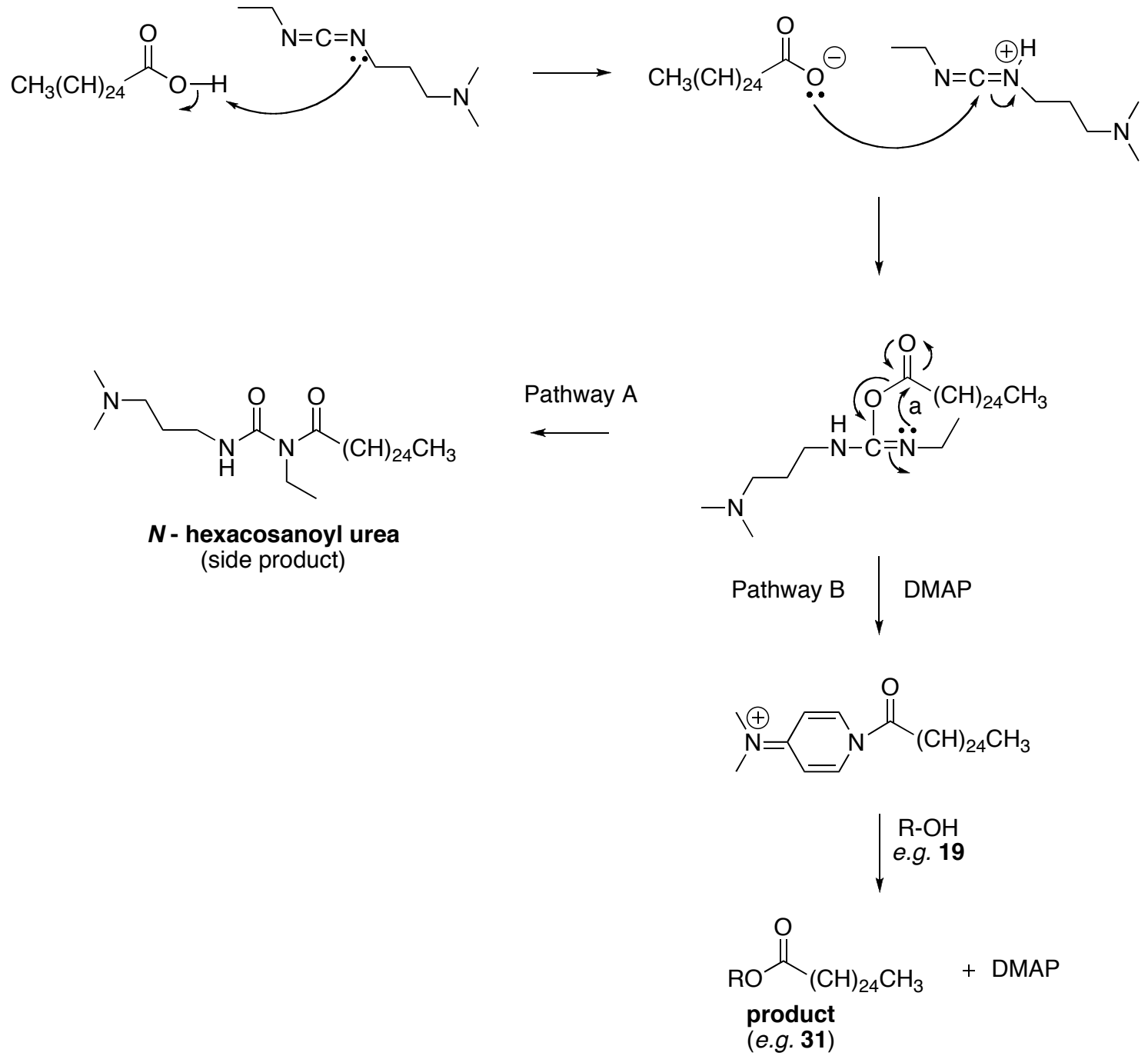

Scheme 8. Mechanism for the formation of $N$-hexacosanoyl urea side-product and desired ester product $\mathbf{3 1}$. 
The ${ }^{1} \mathrm{H}$ NMR spectra of TMS-protected TDE 25 and TMS-protected TME 31 are shown in Figures 4 and 5. In Figure 5, the significant $d d$ resonances at $3.69 \mathrm{ppm}$ and $3.71 \mathrm{ppm}$ both have a coupling constant of $11.7 \mathrm{~Hz}$, which are not seen in Figure 4. These resonances have been assigned to $\mathrm{H}-6 \mathrm{a}^{\prime}$ and $\mathrm{H}-6 \mathrm{~b}^{\prime}$ in one glucose unit of trehalose, further supporting that $\mathbf{3 1}$ is only mono-acylated. Upadhyaya et al. have studied geminal proton-proton couplings in ketal ring systems and reported that exocyclic hydroxymethyl methylene protons have a coupling constant approaching $12 \mathrm{~Hz},{ }^{114}$ which is consistent with the results reported for the TMS-protected TME 31. Similarly, for the $\mathrm{C}_{2}$-symmetrical diester $\mathbf{2 5}$, only one anomeric residue was observed at $4.92 \mathrm{ppm}$ $\left(d, J_{1,2}=2.9 \mathrm{~Hz}, 2 \mathrm{H}, \mathrm{H}-1\right)$, while for the monoester there were two overlapping signals at $4.93 \mathrm{ppm}\left(d, J_{1 / 1^{\prime}, 2 / 2^{\prime}}=3.5 \mathrm{~Hz}, 1 \mathrm{H}, \mathrm{H}-1 / 1^{\prime}\right)$ and $4.92 \mathrm{ppm}\left(\mathrm{d}, J_{1^{\prime} / 1,2^{\prime} / 2}=3.5 \mathrm{~Hz}, 1 \mathrm{H}, \mathrm{H}-\right.$ $1 / 1$ ), respectively. Full characterisation was determined using 2D NMR, and the details of peak assignment are provided in the experimental chapter (Chapter 5). 


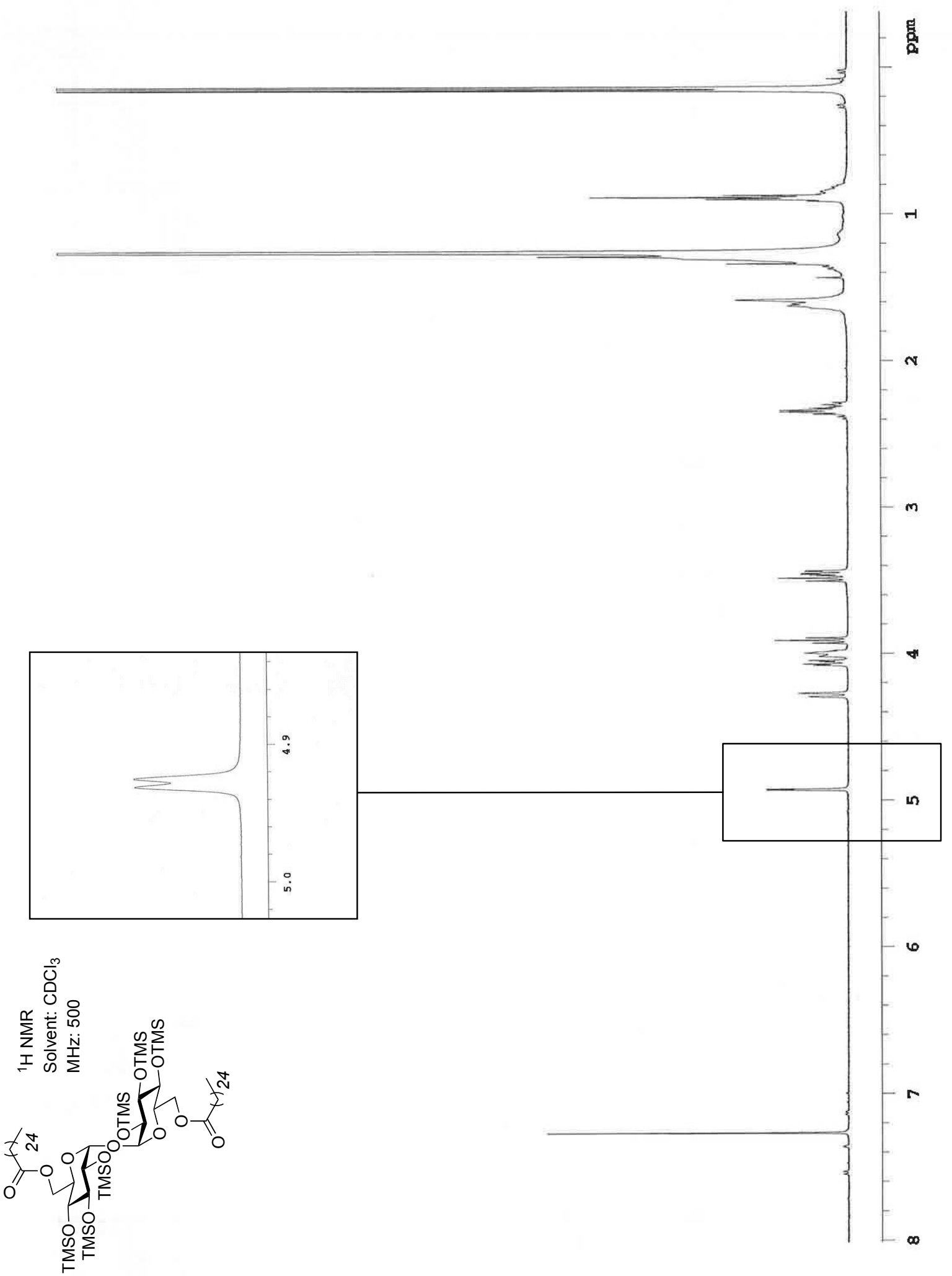

Figure 4. $\quad{ }^{1} \mathrm{H}$ NMR spectra of TMS-protected TDE 25 


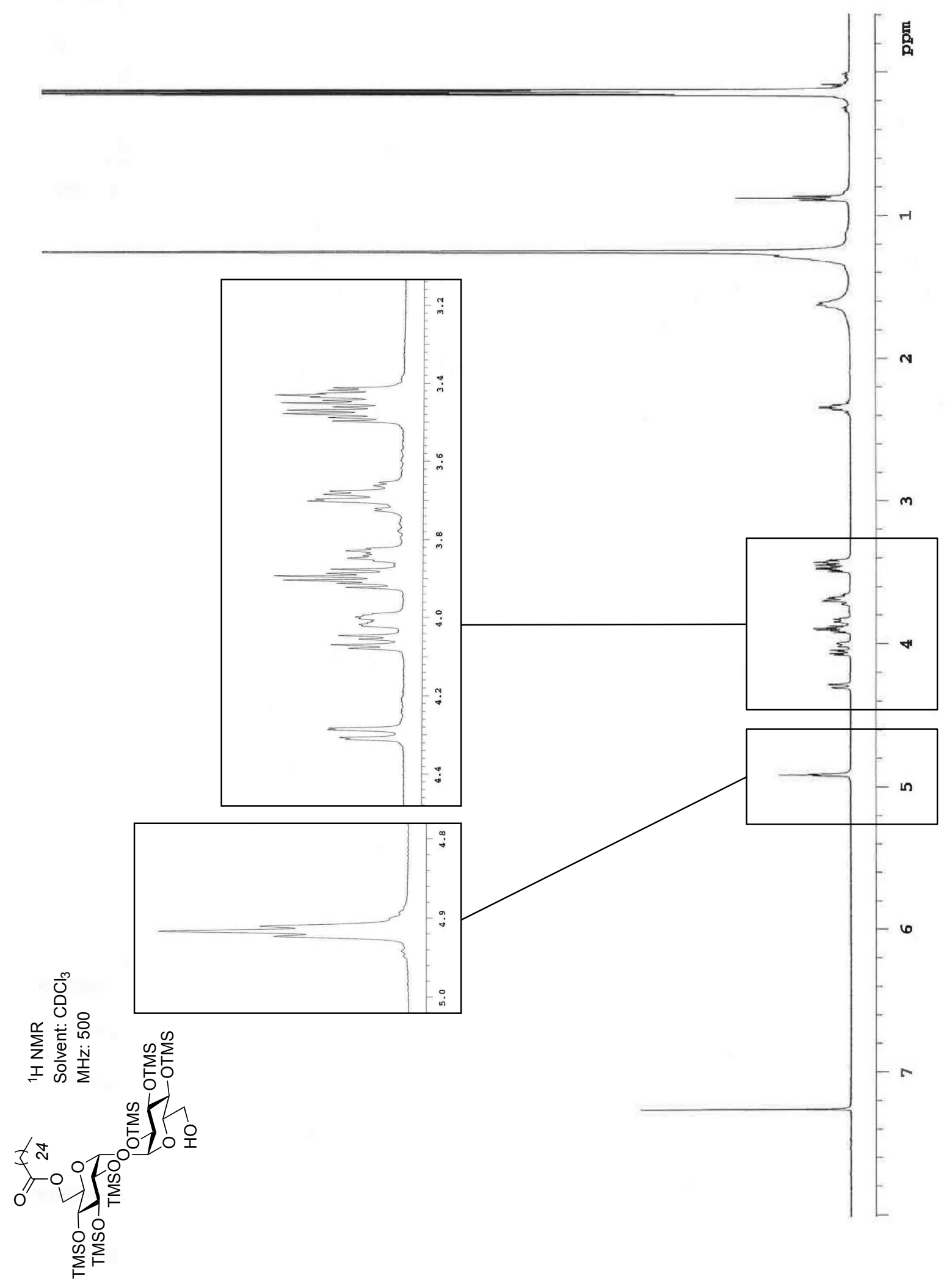

Figure 5. $\quad{ }^{1}$ H NMR spectra of TMS-protected TME 31 


\subsection{Biological evaluation of TMEs}

Following the development of an optimised route for the synthesis of C26 TME 32, the next step was to determine whether these TGs were able to activate M $\varphi$ via Mincle. Accordingly, the ability of these TGs to cause bone-marrow-derived macrophages (BMMs) to produce NO and the cytokine IL-6 was explored. The cellular mediator NO is produced by $\mathrm{M} \varphi$ upon stimulation by bacteria and other pathogens, while the proinflammatory cytokine IL-6 is released in response to specific PAMPs. ${ }^{59,64}$ Thus, by measuring NO and IL-6 production, the activation state of M $\varphi$ s can be deduced.

For the biological assessment of the TGs, I isolated and cultured the M $\varphi$ s for the in vitro testing, while Dr Ashna Khan performed the biological testing (i.e. NO and IL-6 production of the M $\varphi s$ in response to the different trehalose glycolipids). As highlighted previously, the C26 TME 32 and C26 TDE 26 glycolipid used in the biological experiments were synthesised by myself, while the C22 TME and C22 TDE (TDB) glycolipids were synthesised by Dr Ashna Khan.

Mature M $\varphi s$ are difficult to isolate from tissue. Accordingly, there are several ways in which M $\varphi s$ can be obtained. ${ }^{115}$ One way is to use an immortalised M $\varphi$ myeloid cell line, such as the human monocytic cell line THP-1, ${ }^{116}$ however, in the context of my studies, there is no equivalent Mincle $^{-/-}$cell line, that can be used to determine the effect of Mincle on liposome uptake. Another way to obtain $\mathrm{M} \varphi \mathrm{s}$ is through primary $\mathrm{M} \varphi$ culture, whereby cytokines are used to differentiate progenitor cells (monocytes) from the bone marrow into mature Mps in culture. ${ }^{117}$ The main advantage of this method is that a homogenous population of $\mathrm{M} \varphi \mathrm{s}$ in a quiescent state are generated, which are responsive to activation by a stimuli in vitro. ${ }^{115}$ Moreover, Mincle $^{-/-}$BMMs from the corresponding Mincle $^{-/-}$mouse can be generated.

Accordingly, to perform the in vitro experiments discussed in this thesis, I needed to isolate and differentiate the BMMs. To this end, bone marrow was extracted from the 
legs of both wild-type (C57BL/6) and $\mathrm{Mincle}^{-/-}$mice, to obtain the monocytes, and the monocytes were then stimulated with the appropriate cytokines to generate M $\varphi s$ with the required phenotype (Figure 6). As trehalose glycolipids are found on the cell wall of M. tuberculosis, which is a pathogen that causes an inflammatory immune response, the cytokine granulocyte macrophage colony-stimulating factor (GM-CSF) was added to the monocytes so as to generate $\mathrm{M} \varphi \mathrm{s}$ with a 'M1' or 'pro-inflammatory' phenotype. Further details about the process for the generation of BMMs from bone marrow is provided in the experimental section (see Chapter 5). Once generated, the cells were then given to Dr Ashna Khan. A variety of biological assays were performed and a summary of these findings is presented in this thesis. The full details of the experiments performed within the context of the work have recently been published. ${ }^{118}$
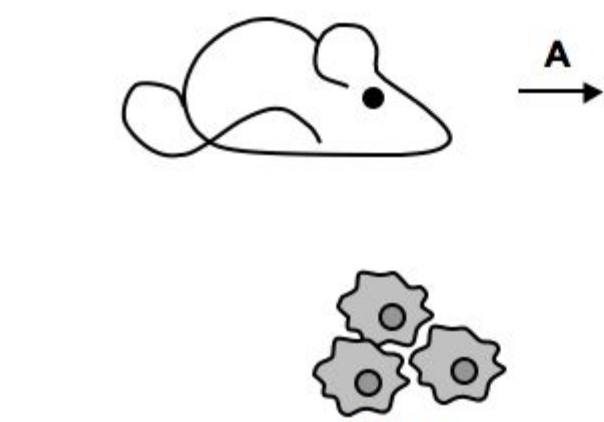

BMMs

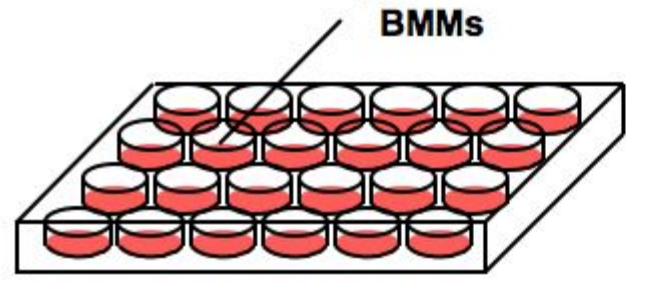

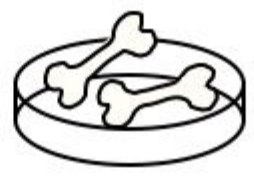

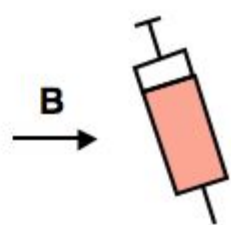

\{\}

0
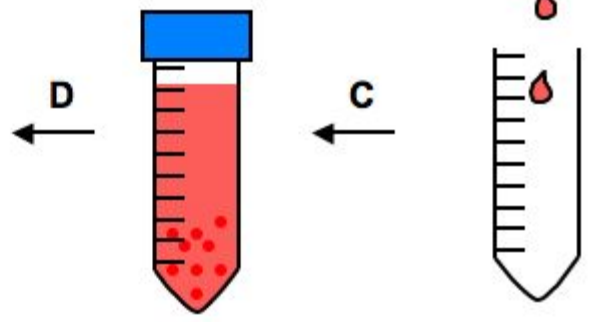

Figure 6. Schematic for Bone Marrow Isolation and BMM Differentiation. A) Sacrifice mice. Use dissection tools to expose the hind legs and remove muscle from the bones. B) Cut the bones at both ends and flush the bone marrow using a syringe and needle into a falcon tube. C) Count the bone marrow cells using a haemocytometer. D) Plate the cells and culture BMMs in vitro. For the purposes of my experiment, the BMMs were cultured using GM-CSF. 
The ability of C26 TME 32 and C22 TME to activate M $\varphi$, as evidenced by production of NO and IL-6 by BMMs after incubation with TGs (TDE or TME) was determined using the Griess assay. ${ }^{59}$ The Griess assay is the most widely accepted classical method for measuring NO production and allows for the quantitative determination of nitrite $\left(\mathrm{NO}_{2}{ }^{-}\right)$ions in various samples using a colourimetric approach. The assay was used by Dr Ashna Khan to determine NO production by BMMs in response to the individual TGs, and later by myself to investigate the ability of liposomes containing the C26-TDE to activate BMMs.

The Griess reagent system consists of $N$-(1-naphthyl)ethylenediamine (33) and sulfanilic acid (34) (Scheme 9). Specifically, the Griess assay detects the amount of nitrite present in a biological sample, with the nitrite itself being formed by the spontaneous oxidation of $\mathrm{NO}$ to $\mathrm{NO}_{2}{ }^{-}$under physiological conditions. ${ }^{119}$ Thus, in the presence of the nitrite anion, sulfanilic acid (34) reacts to form the diazonium salt $\mathbf{3 5}$, which then reacts with amine (33) to yield the azo dye 36, which can then be detected colourimetrically at 548 $\mathrm{nm}$.

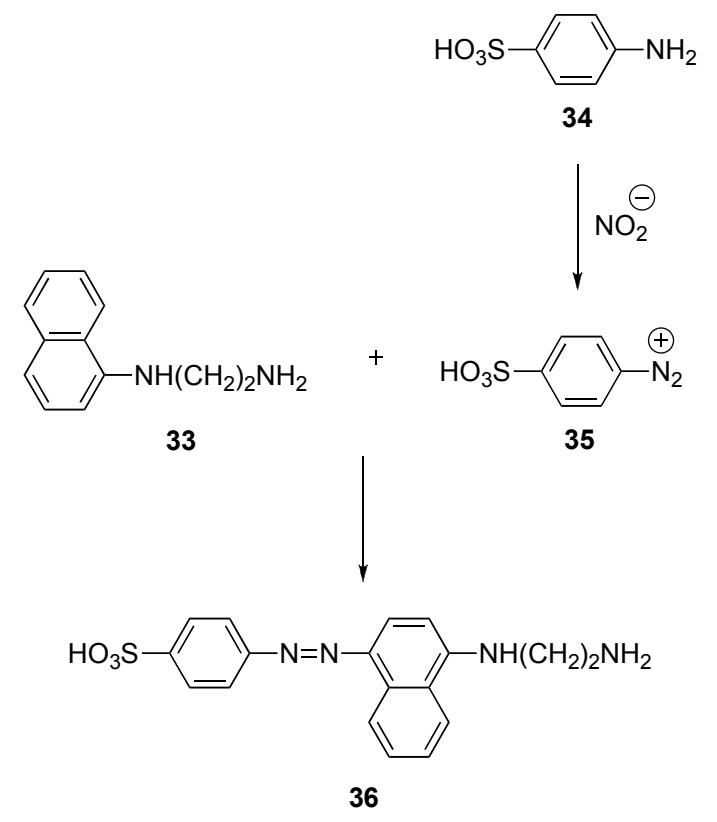

Scheme 9. The chemistry of the Griess assay, that detects NO production by cells. 
As Mincle expression is strongly induced by pro-inflammatory stimuli, including lipopolysaccharide (LPS) and interferon-gamma (IFN- $\gamma$ ), the ability of C26 TME 32 and C22 TME to activate C57BL/6 BBMs primed with IFN- $\gamma$ was first explored (Figure 7). IFN- $\gamma$-primed BMMs were treated with $20 \mu \mathrm{g} / \mathrm{mL}^{-1}$ of C26 TME 32 or C22 TME, and at different time points ( $24 \mathrm{~h}, 48 \mathrm{~h}, 72 \mathrm{~h}$ and $96 \mathrm{~h}$ ) the supernatant was collected and then analysed for NO. As illustrated, Figure 7 A shows that NO production by the BMMs in response to $20 \mu \mathrm{g} / \mathrm{mL}^{-1}$ of C22 (TME) and C26 TME 32 increased over a $96 \mathrm{~h}$ time period, although at a slower rate compared to TDB and LPS, both of which were used as positive controls. At $24 \mathrm{~h}$, the C22 TME, C26 TME 32 and TDB, all induced similar levels of NO, however, after $96 \mathrm{~h}$, the levels of NO were lower for C22 TME and C26 TME 32 (with $32<\mathrm{C} 22$ TME). The effect of glycolipid concentration on NO production was also investigated, as illustrated in Figure 7 B. Here, BMMs were incubated with 20 or $40 \mu \mathrm{g} / \mathrm{mL}$ of the trehalose glycolipids and at $96 \mathrm{~h}$, modest increases in NO were observed with increased glycolipid concentration for both monoesters 32 and C22 TME and for TBD (C22 TDE). These results show that TMEs are able to activate $\mathrm{M} \varphi \mathrm{s}$ and provides the first evidence for this phenomenon. ${ }^{118}$ 
A.

\section{NO Production}

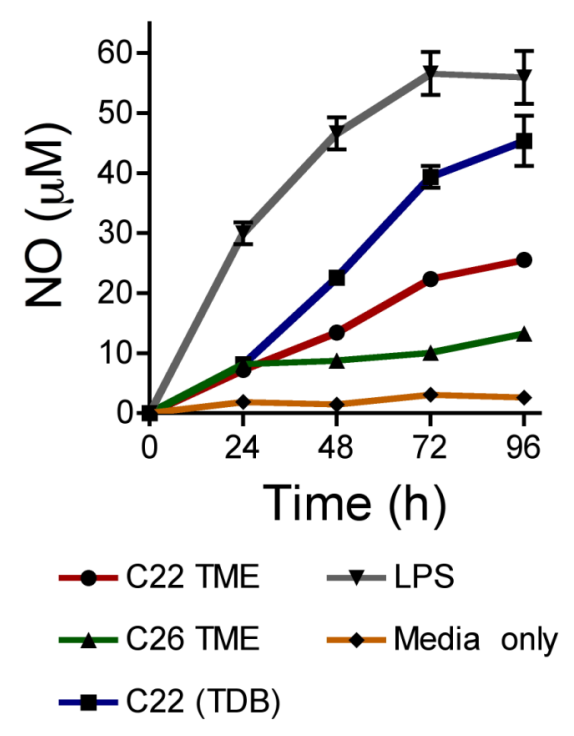

B.

NO Production

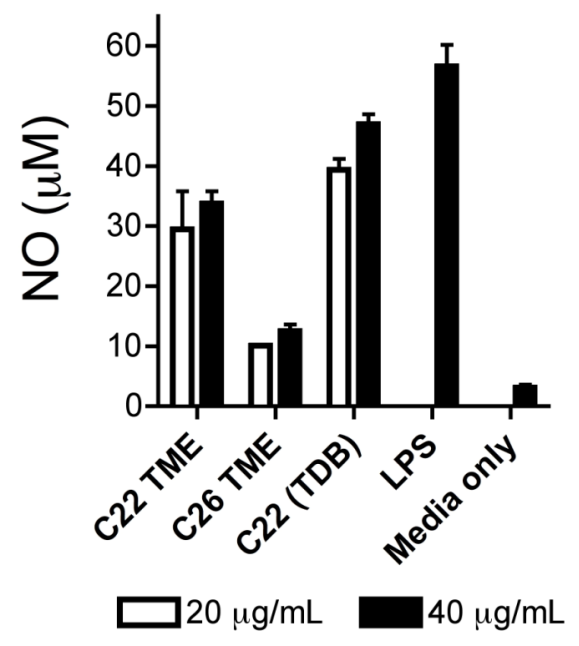

Figure 7. Production of NO by TDB/TME-treated BMMs.A) BMMs were primed with IFN- $\gamma(10 \mathrm{ng} / \mathrm{mL})$ and stimulated with C26 TME 32 or C22 TME $(20 \mu \mathrm{g} / \mathrm{mL})$, with TDB $(20 \mu \mathrm{g} / \mathrm{mL})$ or with LPS $\left(100 \mathrm{ng} / \mathrm{mL}^{-1}\right)$. At 24 , 48,72 and $96 \mathrm{~h}$ the supernatants were analysed for $\mathrm{NO}$ accumulation. B) BMMs were treated with C26 TME 32 or C22 TME $\left(20 \mu \mathrm{g} / \mathrm{mL}^{-} 1\right.$ or 40 $\left.\mu \mathrm{g} / \mathrm{mL}^{-1}\right)$, with TDB, $\left(20 \mu \mathrm{g} / \mathrm{mL}^{-} 1\right.$ or $\left.40 \mu \mathrm{g} / \mathrm{mL}^{-1}\right)$ or with LPS (100 $\mathrm{ng} / \mathrm{mL}^{-1}$ ), and NO production was determined at $96 \mathrm{~h}$. For both graphs, the means and SDs of triplicate samples from representative experiments performed in duplicate are shown.

Having established that C26 TME 32 and C22 TME activate BMMs with priming, investigations into whether TMEs were able to activate $M \varphi s$ in the absence of priming were then conducted. Lang and co-workers previously demonstrated that TDB led to the induction of NO by BMMs, although at a lower level than when BMMs were primed by IFN- $\gamma .{ }^{59}$ Thus, the abilities of C22 TME and C26 TME 32 to activate Mincle ${ }^{-/-}$BMMs without IFN- $\gamma$ priming were investigated, with use of LPS and IFN- $\gamma$ as positive controls and media alone as a negative control. From the studies performed in our group, however, it was determined that in the absence of IFN- $\gamma$ priming there was negligible 
NO production by the BMMs in response to either the mono- or di- esters over the $96 \mathrm{~h}$ time course (Figure 8 A). ${ }^{118}$ This result was surprising because Lang and co-workers had previously observed NO production by TDB, ${ }^{59}$ however, differences in the ability of TDB to activate M $\varphi$ s have been noted previously. ${ }^{120}$ The mono- and diesters, however, both led to the production of IL-6 by BMMs (Figure $8 \mathrm{~B}$ ), thereby demonstrating that Mps can be activated without the need for priming. This is important as it demonstrates how the TGs in liposomes can be used to target M $\varphi$ s. If priming were needed, then it may mean that additional 'priming' agents (e.g. IFN- $\gamma$ or LPS) may need to be added to the liposome before performing any in vivo studies. Finally, it should also be noted that the TMEs were also able to active BMMs in a Mincle-dependent manner, (Figure 8 B). ${ }^{118}$ 
A.

NO Production

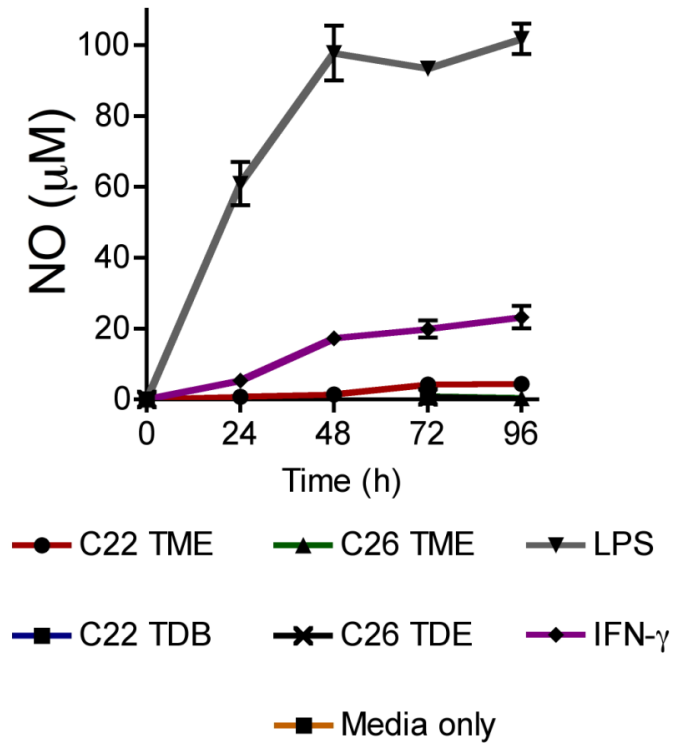

B.

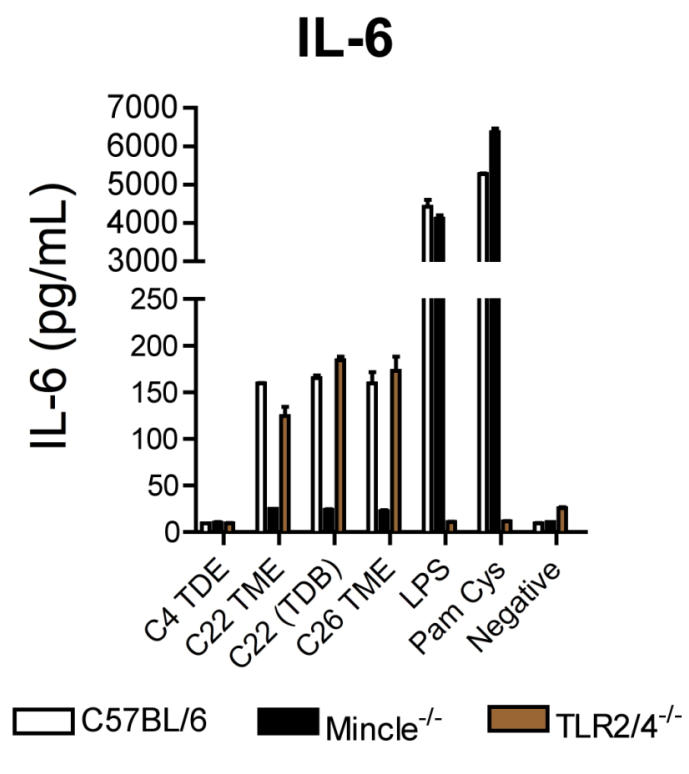

Figure 8. BMM activation without priming by IFN- $\gamma$. A) NO, and B) IL-6 production by TDE/TME-treated BMMs derived from $\mathrm{Mincle}^{-/}$, TLR2 $/ 4^{-/-}$and C57BL/6. BMMs were primed with IFN- $\gamma\left(10 \mathrm{ng} / \mathrm{mL}^{-1}\right)$ and stimulated with TMEs 32 or C22 TME, TDB, TDE 26 at 40 $\mu \mathrm{g} / \mathrm{mL}^{-1}$, or with LPS or PamCys at $10 \mathrm{ng} / \mathrm{mL}^{-}$, and NO or IL-6 were measured after $72 \mathrm{~h}$. For both graphs the means and SDs of triplicate samples from representative experiments performed in duplicate are shown.

In experiments using $\mathrm{Mincle}^{-/-}$mice (in the presence of priming), neither NO production $^{118}$ or IL-6 production (Figure 8 B) was observed for either the monoesters 32 and C22 TME, or the diesters 26 or TDB (positive control), yet activity was observed when using the wild-type $(\mathrm{C} 57 \mathrm{BL} / 6)$ or TLR $2 / 4^{-/-}$mice. Taken as a whole, these findings support the recent crystallographic evidence provided by Feinberg et al. and Furukawa et al., ${ }^{69,70}$ which suggests that there is one major binding groove for a trehalose glycolipid ligand. The studies, however, also show in a functional assay that TMEs can activate M $\varphi s$. Thus, it has been demonstrated that there is the potential to use 
TMEs in the generation of liposomes as the TMEs appear to have similar biological activity to their diester counterparts. For the purposes of my research, however, I continued on with the synthesis and study of liposomes containing the C26 TDE to determine the experimental conditions, (e.g. liposome formulation, loading and glycolipid concentration), that are best suited for studying TG-liposome uptake by M $\varphi$ s.

\subsection{Conclusion}

In conclusion, the $\mathrm{C} 26$ trehalose monoester 32 containing a single acyl chain, was synthesised in good overall yield and shown to activate BMMs without the need for priming. This is the first time that a TME has been found to activate M $\varphi s$, and by demonstrating that only one ester functionality is required, a new subclass of Minclebinding ligands has been discovered. These studies provide important background for the rational design of other Mincle agonists and indicate that liposomes containing TMEs may provide another avenue by which to better target M $\varphi s$. 


\section{CHAPTER 3:}

\section{FORMATION AND BIOLOGICAL EVALUATION OF TG-INCORPORATED LIPOSOMES}

\subsection{Overview}

Having successfully synthesised the C26 TDE and TME, the next objective of this research project was to prepare liposomes containing these trehalose glycolipids and to determine whether the presence of the glycolipids influences the activation of M $\varphi$. To this end, liposomes containing the C26 TDE at varying concentrations, as well was liposomes without the trehalose glycolipids (as a control), needed to be prepared.

While there has been much research on the synthesis of liposomes as drug delivery vehicles, the exact liposome characteristics for optimal uptake by M $\varphi$ s depends on multiple factors including the phospholipids charge. ${ }^{97}$ Although, it is generally recognised that negatively charged lipids [e.g. phosphatidylserine (PS) and phosphatidylglycerol (PG)] are preferentially recognised by $\mathrm{M} \varphi \mathrm{s}^{101}$ phosphatidylcholine (PC) was selected for its neutral charge. Neutrally charged liposomes were desirable in this research, as it would allow the effect of TDE on phagocytosis by $\mathrm{M} \varphi s$ to be better studied. Accordingly, the liposomes were prepared using PC, 69\%, Cholesterol 30\%, and the C26 TDE, with the ratio of PC:Cholestrol added being approximately 3:1, and with different amounts of the C26 TDE being added to the solution. Typically, concentrations of other cell targeting ligands [e.g. the single mannose head group ligand, monomannose-dipalmitoylphosphatidylethanolamine (8[carboxy-2-(1,2-dihexadecanoyl-sn-glycero-3-sodio-phospho)ethanolamido]octyl $\alpha$-Dmannopyranoside) or branched tri-mannose head group ligand, trimannosedipalmitoylphosphatidylethanolamine (8-[carboxy-2-(1,2-dihexadecanoyl-sn-glycero-3- 
sodio-phospho)ethanolamido]octyl

( $\alpha$-D-mannopyranosyl)- $(1 \rightarrow 3)-[(\alpha-D-$ mannopyranosyl)-(1 $\rightarrow 6)$ ]- $\alpha$-D-mannopyranoside] are added at loadings of $0-20 \%$ of the total lipid weight, ${ }^{121}$ and thus, the initial amount of TDE to be incorporated was from $4-16 \%$ of the total lipid weight of the liposome. The molecular (lipid) weight for an empty liposome (i.e. the number of moles of phosphatidylcholine and cholesterol) was calculated, and loading at $4 \%, 8 \%$ and $16 \%$ of TDE was determined by calculation of these respective percentages from this total lipid weight (See Chapter 5 for representative calculations). Specifically, the work in this MSc thesis focused on the synthesis of 'empty' liposomes (i.e. liposomes without Mps depleting drugs or fluorescent material inside the liposome), with the results from these studies informing future work for the formulation of liposomes containing cargo and/or fluorescent dyes.

\subsection{Preparation of Liposomes}

To prepare the required liposomes, the dried-lipid film hydration method, as previously described by Van Rooijen et al. ${ }^{96}$ was used. The detailed protocol is provided in the experimental section and is briefly outlined here. Accordingly, PC, cholesterol and the C26 TDE were dissolved in a minimum volume of chloroform in a glass round-bottom flask and the chloroform was slowly removed under reduced pressure using a rotary evaporator so as to deposit a thin lipid film on the wall of the flask. Next, PBS (pH 7.4) was added to give a target TDE lipid concentration of $60 \mu \mathrm{M}$, and the resulting emulsion was dispersed by gentle rotation for 1 hour on the rotatory evaporator, followed by brief sonication ( $3 \mathrm{~min}$ ), and resting for 2 hours at room temperature. Before use in in vitro assays, the liposome suspension was homogenised by gentle shaking before adding to cultures to achieve a homogeneous distribution of the liposomes in suspension. 


\subsection{Characterisation of Liposomes: Percentage of C26 TDE incorporated into liposomes}

The percentage of TDE incorporated into liposomes was examined using the phenol-

sulfuric acid method to analyse carbohydrate content. ${ }^{122}$ Although there are many colourimetric methods to analyse carbohydrate content, most use phenol and sulfuric acid and mainly differ in the concentration of reagents used. The phenol-sulfuric acid method is easy and reliably measures the neutral sugar content in oligosaccharides, proteoglycans, glycoproteins, and glycolipids. ${ }^{123}$

The phenol-sulfuric acid assay works via the acid catalysed hydrolysis of glycosidic linkages followed by the reaction of phenol at the reducing terminus to give an adduct that can be detected colourimetrically. As illustrated (Scheme 10), reaction of the oligosaccharide (I) with $\mathrm{H}_{2} \mathrm{SO}_{4}$ gives lactol (II), which subsequently undergoes nucleophilic attack by phenol (III) at the anomeric centre to give the alcohol (IV). This adduct then undergoes an $\mathrm{E} 1 \mathrm{cB}$ reaction to yield the coloured adduct $(\mathbf{V})$, which is detectable at $490 \mathrm{~nm}$. 


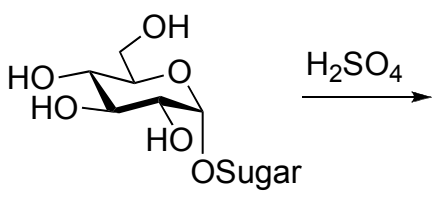

I

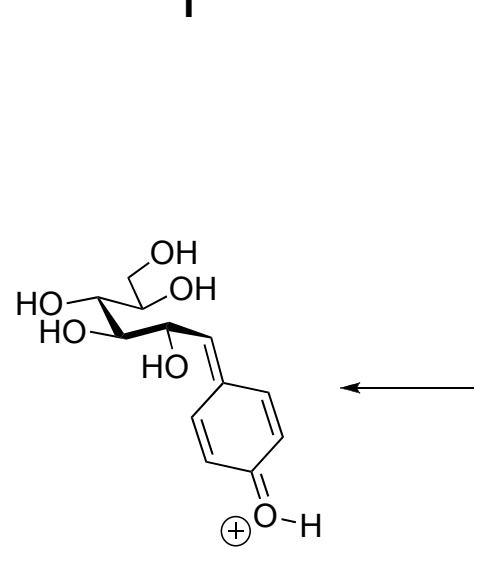

V

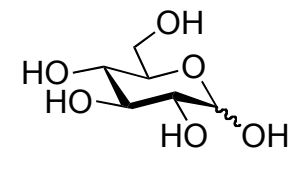

II

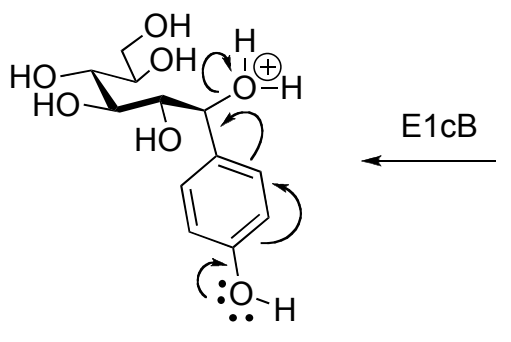

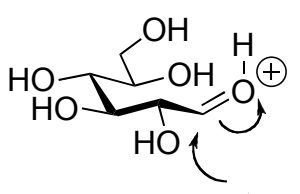
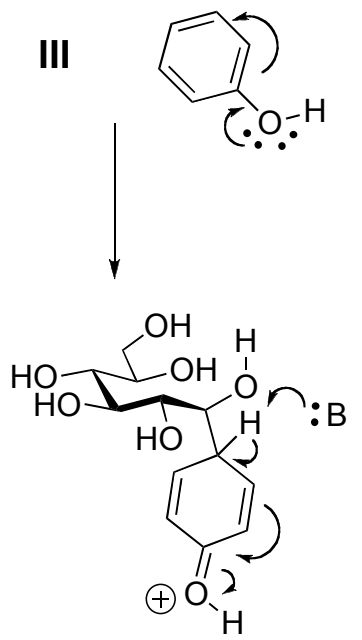

IV

Scheme 10. The mechanism of the phenol-sulfuric acid colourimetric assay.

Before the amount of C26 TDE in the liposomes was determined, a calibration curve for a sugar standard needed to be determined. In this MSc project, the calibration curve was derived using $\alpha, \alpha^{\prime}$-trehalose as the standard using concentrations of $0.1 \mathrm{mg} / \mathrm{mL}$ to 0.5 $\mathrm{mg} / \mathrm{mL}$ (Figure 9), with the measurements being performed in triplicate and a representative one of three experiments shown. It was assumed that for $\alpha, \alpha^{\prime}$-trehalose, each molecule of trehalose reacts to form two molecules of dye. As illustrated, there is some variability with the measurements, however, the larger errors are observed at higher concentrations (i.e. above the concentration anticipated for C26 TDE loading in the liposomes). Moreover, for the purposes of the forthcoming biological assessment, it is an indication of the amount of C26 TDE incorporated into liposomes is provided rather than an absolute value. 


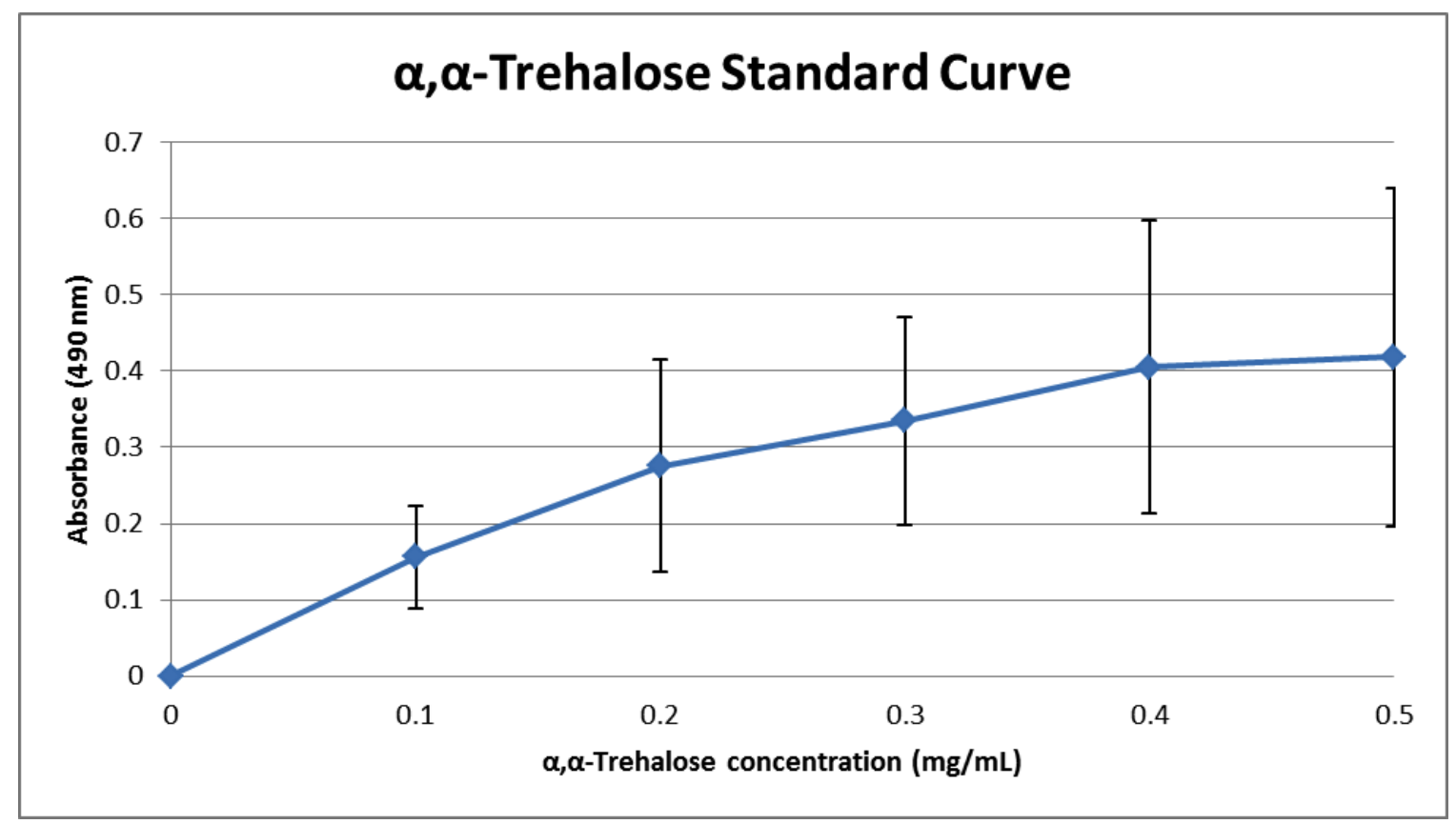

Figure 9. Standard curve of $\alpha, \alpha^{\prime}$-trehalose in distilled water. For the graph, the means and SE of triplicate samples from representative experiments performed in duplicate are shown.

Having determined the calibration curve using $\alpha, \alpha^{\prime}$-trehalose, those liposomes containing the C26 TDE were then analysed for their glycolipid content. To this end, the liposomes were prepared as previously described ${ }^{96}$ and then were pelleted by centrifugation at 10,000 RPM for $10 \mathrm{~min}$ and the PBS was removed using a glass pipette without disrupting the pellet. The liposomes were then resuspended in distilled water to an anticipated concentration of $0.5 \mathrm{mg} / \mathrm{mL}$ of trehalose diester, and titrated down in 2 fold dilutions in triplicate into a 96-well plate and sulfuric acid added to each sample, followed immediately by $5 \%$ phenol solution. The $96-$ well plate was then incubated for 5 mins at room temperature before the absorbance was measured on a microtiter plate reader at $490 \mathrm{~nm} .{ }^{123}$ The absorbance of the unknown liposome suspension at $490 \mathrm{~nm}$ was then compared to that of the standard curve to give a percentage of C26 TDE incorporation (see Experimental section for the procedure). Accordingly, it was determined that the amount of C26 TDE incorporated into the liposomes was less than the total amount of TDE added to the solution (Table 2). In the target 4\% TDE mol. 
weight doping, only $13 \%$ of TDE was incorporated to give an actual TDE mol. weight doping of $0.5 \%$, while, in the target $8 \%$ and $16 \%$ TDE mol. weight doping, higher percentages $(28 \%$ and $40 \%)$ of TDE was incorporated to give an actual TDE mol. weight doping of 2.3 and $6.3 \%$, respectively. From herein on, the percentage of actual C26 TDE incorporation is displayed (i.e. when discussing the biological results).

Table 2. Percentage of C26-TDE incorporated into liposome stock suspensions.

\begin{tabular}{c|c|c}
$\begin{array}{c}\text { Target } \\
\text { TDE mol. weight } \\
\text { doping (\%) }\end{array}$ & $\begin{array}{c}\text { Actual } \\
\text { TDE mol. weight } \\
\text { doping (\%) }\end{array}$ & $\begin{array}{c}\text { Percentage of TDE } \\
\text { incorporated into } \\
\text { liposome (\%) }\end{array}$ \\
\hline 4.0 & 0.5 & 13 \\
8.0 & 2.3 & 28 \\
16.0 & 6.3 & 40
\end{tabular}

\subsection{Characterisation of Liposomes: Size and distribution}

The sizes of the liposomes were measured on the Zetasizer Nano ZS using dynamic light scattering (DLS). This technique measures the time-dependent fluctuations in the intensity of scattered light, which occur because the particles are undergoing Brownian motion. ${ }^{124}$ Size measurements were made on the neat liposome suspension and also in samples, which were diluted 1 in 4 with PBS. The results from this work revealed a heterogeneous mixture of liposomes with an average size of $98 \pm 31 \mathrm{~nm}$ for the empty phosphatidylcholine liposomes and an average size of $133 \pm 37 \mathrm{~nm}$ for the C26 TDE containing liposomes. 


\subsection{Ability of Liposomes to Activate Macrophages}

The overall objective of this research was to determine whether liposomes containing TGs are preferentially phagocytosed by M $\varphi s$. In this MSc thesis, I will describe the effect of $\mathrm{C} 26 \mathrm{TDE}$ containing liposomes on $\mathrm{M} \varphi$ activation to determine which liposome characteristics lead to $M \varphi$ activation. As activated $M \varphi s$ are typically more phagocytic, such a readout will provide an indirect measure of liposome uptake. To confirm the preferential uptake of liposomes containing TGs, however, the liposome experiments will need to be repeated using liposomes containing a fluorescent dye, with uptake being measured by flow cytometry. Flow cytometry experiments are more time consuming and costly, and thus were not initially used.

To determine $M \varphi$ activation, the ability of the M $\varphi s$ to produce NO was explored. As described in Chapter 2, NO is the radical responsible for the cytotoxic effects of $\mathrm{M} \varphi \mathrm{s}$ and is commonly produced by activated $\mathrm{M} \varphi s .{ }^{59}$ Once again, the NO production by $\mathrm{M} \varphi \mathrm{s}$ was determined using the Griess Assay, ${ }^{59}$ however for the liposome studies discussed in Chapter Three, I performed all biological experiments.

\subsubsection{Titration of Phosphatidylcholine Liposomes}

Initially, I wanted to determine whether the lipids phosphatidylcholine (PC) and cholesterol that make up the majority of the liposome had an effect on the activation of BBMs. Accordingly, the BBMs were cultured from wildtype (C57BL/6), as well as Mincle $^{-/-}$mice as described in Chapter Two, and on day 10 were subjected to $\mathrm{PC} /$ Cholesterol liposomes with varying concentrations of PC. The supernatant was then collected at 24, 48 and 72 hour time points and NO was measured. The positive control in these experiments was lipopolysaccharide (LPS), a known activating agent for $\mathrm{BMMs},{ }^{125}$ while the negative control was PBS alone. 
Thus, a titration experiment was performed and the concentration of PC was decreased from $800 \mu \mathrm{M}$ to $6.25 \mu \mathrm{M}$ in both C57BL/6 (Figure $10 \mathrm{~A}$ ) and Mincle ${ }^{-/-}$BMMs (Figure $10 \mathrm{~B})$. The $\mathrm{Mincle}^{-/-}$mice were used to determine if there was any substantial difference in the phenotype of BMMs from the Mincle knock-out mice, as future studies include determining whether the Mincle receptor influences the uptake of trehalose containing glycolipids. My experiments demonstrated that the BMMs were activated in a concentration-dependent manner with a modest increase in NO production being observed with increasing concentration of PC in liposomes in both C57BL/6 and in $\mathrm{Mincle}^{-/-}$mice, for a liposome concentration with $\geq 200 \mu \mathrm{M}$ of PC. Initially it was surprising that the $\mathrm{PC} /$ cholesterol liposomes led to NO production by BMMs as these types of liposomes are generally considered to be biologically inert. Accordingly, a more thorough review of the literature was undertaken to determine whether others have observed a concentration dependence of PC on biological activity. 
A.

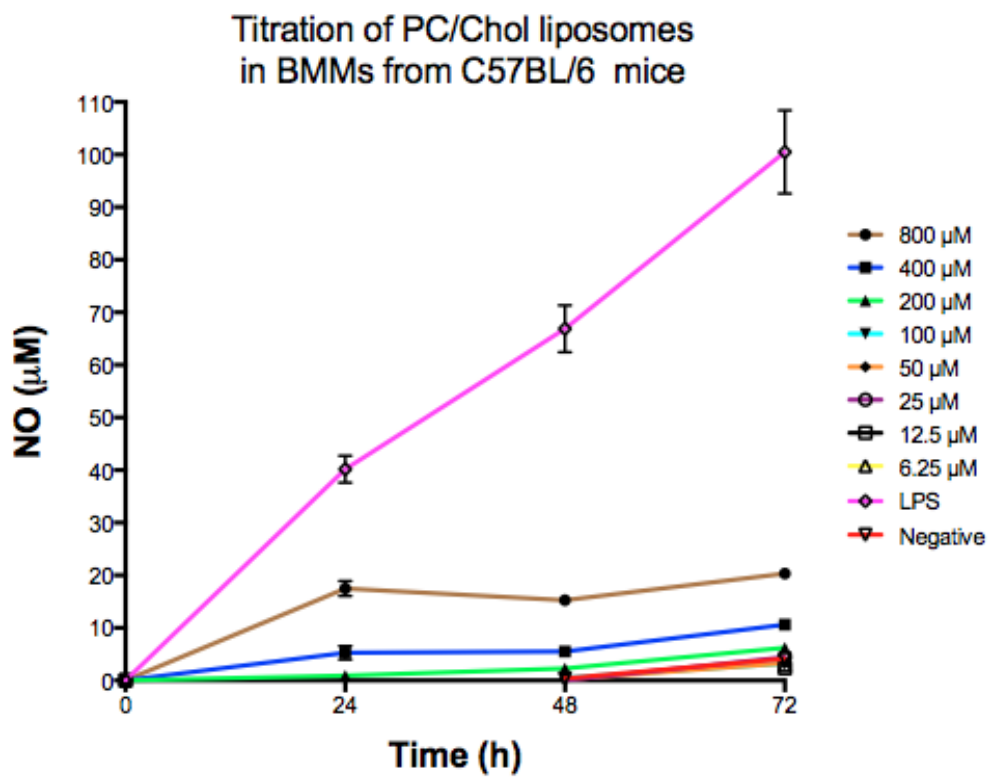

Titration of PC/Chol liposomes

in BMMs from Mincle ${ }^{-t-}$ mice

B.

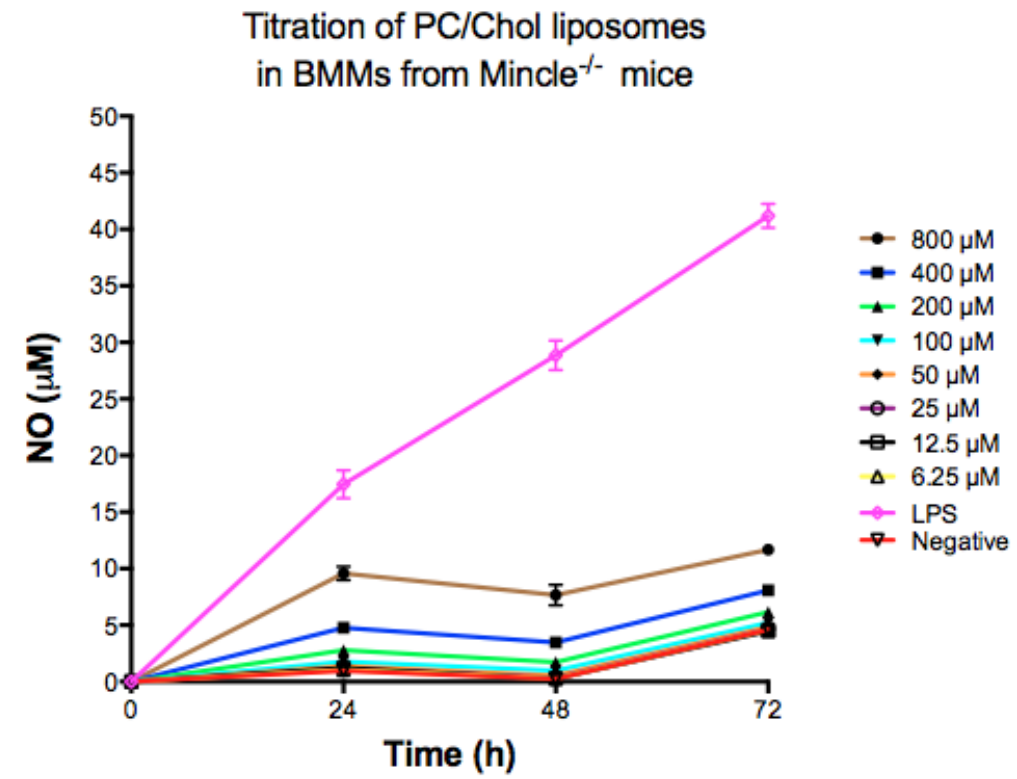

Figure 10. Titration of PC/cholesterol liposomes in BMMs. A) NO production by empty liposome-treated BMMs derived from $\mathrm{C} 57 \mathrm{BL} / 6$ mice, and $\mathrm{B}$ ) Mincle $^{-/-}$BMMs were primed with IFN- $\gamma\left(10 \mathrm{ng} / \mathrm{ml}^{-1}\right)$ and stimulated with a suspension of $\mathrm{PC} /$ cholesterol liposomes alone at various concentrations of PC, or with LPS $\left(10 \mathrm{ng} / \mathrm{ml}^{-1}\right)$ or PBS only. At 24, 48 and $72 \mathrm{~h}$ the supernatants were analysed for NO accumulation. For both graphs the means and SDs of triplicate samples from representative experiments performed in duplicate are shown. 
Previously, Grando et al. conducted in vitro experiments investigating the effects of saturated and unsaturated fatty acid-rich PC on rat M $\varphi$ activity. ${ }^{126}$ Here, LPS-stimulated $\left(10 \mathrm{ng} / \mathrm{mL}^{-1}\right)$ peritoneal $\mathrm{M} \varphi \mathrm{s}$ were incubated with linoleic acid-rich-PC (unsaturated PC) and distearoyl-PC (saturated PC) and NO production was measured. In these studies, it was revealed that peritoneal Mps treated with unsaturated PC at $32 \mu \mathrm{M}$ and $64 \mu \mathrm{M}$ concentrations led to a decreased production of NO in a dose-dependent manner compared to the positive control (LPS) by $30.4 \%$ and $46.4 \%$, respectively. However, saturated PC at those same concentrations did not affect NO production of LPS stimulated M $\varphi s .{ }^{126}$ The authors then studied the effect of the nature of the fatty acid on $\mathrm{M} \varphi$ viability. Accordingly, M $\varphi$ s were incubated with various concentrations of saturated PC and unsaturated PC and cell viability was determined by the colourmetric MTT (3[4,5-dimethylthiazol-2-yl]-2,5 diphenyl tetrazolium bromide) assay. ${ }^{126}$ Saturated PC and unsaturated $\mathrm{PC}$ were added to the $\mathrm{M} \varphi$ culture at concentrations up to $256 \mu \mathrm{M}$ for $24 \mathrm{~h}$, and the absorbance was measured at $570 \mathrm{~nm}$. The $\mathrm{M} \varphi \mathrm{s}$ cultured for $2 \mathrm{~h}$ at any of the PC concentrations tested preserved cell viability, however, M $\varphi$ s incubated for $24 \mathrm{~h}$ with 256 $\mu \mathrm{M}$ of saturated PC suffered a significant decrease in cell viability. ${ }^{126}$ This result provides evidence of the cytotoxic effect of $\mathrm{PC}$ at higher concentrations.

In light of the findings by Grando et al., the composition of the L- $\alpha-\mathrm{PC}$ manufactured by Sigma Aldrich was investigated. The MSDS for this product (P 3556, CAS 8002-43-5) states that there is a fatty acid content of approximately $33 \%$ of palmitic (16:0), $13 \%$ stearic (18:0), 31\% oleic (18:1) and 15\% linoleic (18:2), with other fatty acids being observed in minor amounts. Thus, the PC used for these experiments had a 1:1 ratio of saturated PC to unsaturated PC. In the experiments that I performed, NO production was observed at PC concentrations $\geq 200 \mu \mathrm{M}$, which may be due to the toxicity of the PC and cell death leading to NO production by M $\varphi s$ (with M $\varphi$ s engulfing dead cells leading to NO production, which is a known phenomenon). The difference between my work and Grando et al. was that they stimulated M $\varphi$ s with LPS, which led to the production of NO (as seen in my own experiment) and therefore, $\mathrm{M} \varphi$ death would lead to a drop in overall levels of NO. 
However, as observed in my experiments (Figure 11), there also appears to be some non-specific cell death of the M $\varphi s$ after $48 \mathrm{~h}$, as observed by an increase in NO production in the negative control. Moreover, the effect is more pronounced in the Mincle $^{-/-}$BMMs. These experiments (Figure $10 \mathrm{~A}$ and $10 \mathrm{~B}$ ) were repeated several times ( $>3$ independent experiments), and with samples at all time points taken in triplicate, and thus, the results presented are from representative experiments and are reproducible. Accordingly, it seems that the most reliable time point to take in this assay is $48 \mathrm{~h}$, which leads to NO production by M $\varphi$ s without any associated non-specific cell death. Similar trends were also observed in titration experiments in wildtype and Mincle $^{-/-}$ BMMs using solutions with different concentrations of PC only (data not shown), however, titrations of cholesterol alone were not performed as the cholesterol was insoluble in PBS, the buffer used in the liposomal formulations.

While no non-specific cell death was observed in the TME studies presented in Chapter 2 at $72 \mathrm{~h}$ and even at $96 \mathrm{~h}$, (with the negative controls showing no NO production at these time points), others have observed non-specific cell death of M $\varphi$ s post the $48 \mathrm{~h}$ time period. For example, when exploring the liposomal cholesterol delivery to dissect the innate immune arm of M $\varphi$ s during Leishmania infection, ${ }^{127}$ Ghosh et al. studied $\mathrm{M} \varphi \mathrm{s}$ that were uninfected (normal) or infected with L.donovani and then treated them with PC and cholesterol-containing liposomes, together with an Acyl-CoA:cholesterol acyltransferase (ACAT) inhibitor (at $20 \mu \mathrm{g} / \mathrm{mL}$ ) in vitro. At $24 \mathrm{~h}$, NO generation had reached a maximum, however after this time period, NO levels suddenly decreased in both the infected and non-infected M $\varphi$ s, which was due to cell death. ${ }^{127}$ In these studies, the negative controls were uninfected (normal) and infected M $\varphi s$ that was left untreated, and no NO production was observed for these controls over the $48 \mathrm{~h}$ time course. Thus, it appears that non-specific cell death of M $\varphi$ s when subjected to liposomes can occur after longer time periods, and indeed, in my own work, levels of NO tend to rise for liposomes with higher concentrations of PC from the $0-24 \mathrm{~h}$ time period, with levels of NO production plateauing from the $24-48 \mathrm{~h}$ time period, before showing a slight increase. Perhaps the difference between the two general types of experiments (i.e. 
individual TGs verses liposome formulation) is due to the fact that the liposomes, as compared to the individual TGs, are more readily phagocytosed and thus, the immune response peaks earlier, with the later rise in NO production (post $48 \mathrm{~h}$ ) being due to nonspecific cell death.

Similarly, König et al. analysed the proliferative capacity of BMMs after treatment with $\mathrm{PC}$ and cholesterol liposomes at $72 \mathrm{~h}$ in vitro and observed some non-specific death of $\mathrm{M} \varphi \mathrm{s}$ in response to liposomes and media alone. ${ }^{128}$ In these studies, empty liposomes were found to significantly reduce BMM proliferation compared to the control (PBS) and cell cycle analysis showed that empty liposome treatment caused cell cycle arrest at the G1/S boundary. ${ }^{128}$ Additionally, BMM apoptosis was also investigated and the degree of apoptotic cells was higher with empty liposome treatment than with the PBS control $(13 \%$ vs. $7 \%),{ }^{128}$ however, it should also be stressed that there was some nonspecific death of M $\varphi$ s in PBS alone. Thus, taken as a whole, care needs to be taken when measuring $\mathrm{M} \varphi$ activation at the later time points as non-specific cell death can occur. The effectiveness/appropriateness of an assay can be assessed by analysing the negative control to check for the activation status of the M $\varphi s$ in the absence of any stimulatants, however, as non-specific cell death has been observed for empty (unfunctionalised) liposomes, then the most appropriate time point to consider appears to be $48 \mathrm{~h}$. In the context of my empty liposome studies, it is also important to note that all liposomal formulations and PC solution alone were checked for LPS contamination by using endotoxin detection kits. ${ }^{1}$ In all instances, no endotoxin was observed.

In summary, my results have highlighted that to explore NO production as a consequence of TG incorporation into liposomes, the amount of PC used should stay below $200 \mu \mathrm{M}$ to preserved cell viability and NO should be measured at the circa $48 \mathrm{~h}$ time point to account for potential $\mathrm{M} \varphi$ activation due to non-specific cell death. However, it may still be useful to measure NO activation at the $72 \mathrm{~h}$ time point and then

\footnotetext{
${ }^{1}$ All liposome suspensions were tested to be endotoxin-free at a sensitivity of $0.125 \mathrm{EU} / \mathrm{mL}$ with an endotoxin-kit (Pyrotell, Limulus amebocyte lysate) according to the manufacturer's instructions.
} 
assess the data for its relevance, especially if the negative control does not show nonspecific cell death. (i.e. no NO production is observed).

\subsubsection{Activity of C26-TDE containing Liposomes: TG and Liposome concentration}

From my previous analyses, it was determined that liposomes with a PC concentration of less than $200 \mu \mathrm{M}$ did not lead to significant NO production by BMMs. Accordingly, in the subsequent liposome studies aimed at investigating how liposomes containing the C26 TDE affect the activation of BMMs, I used a $105 \mu \mathrm{M}$ concentration of PC. In these C26 TDE liposome studies, as illustrated in Figure 11, both the amount of liposome material added to the $\mathrm{M} \varphi$ and the percentage loading of the C26-TDE in the liposome was explored. As mentioned previously, the percentage incorporation of TDE into the liposomes is the approximate amount of actual TDE incorporated, as determined by the phenol-sulfuric acid test. 


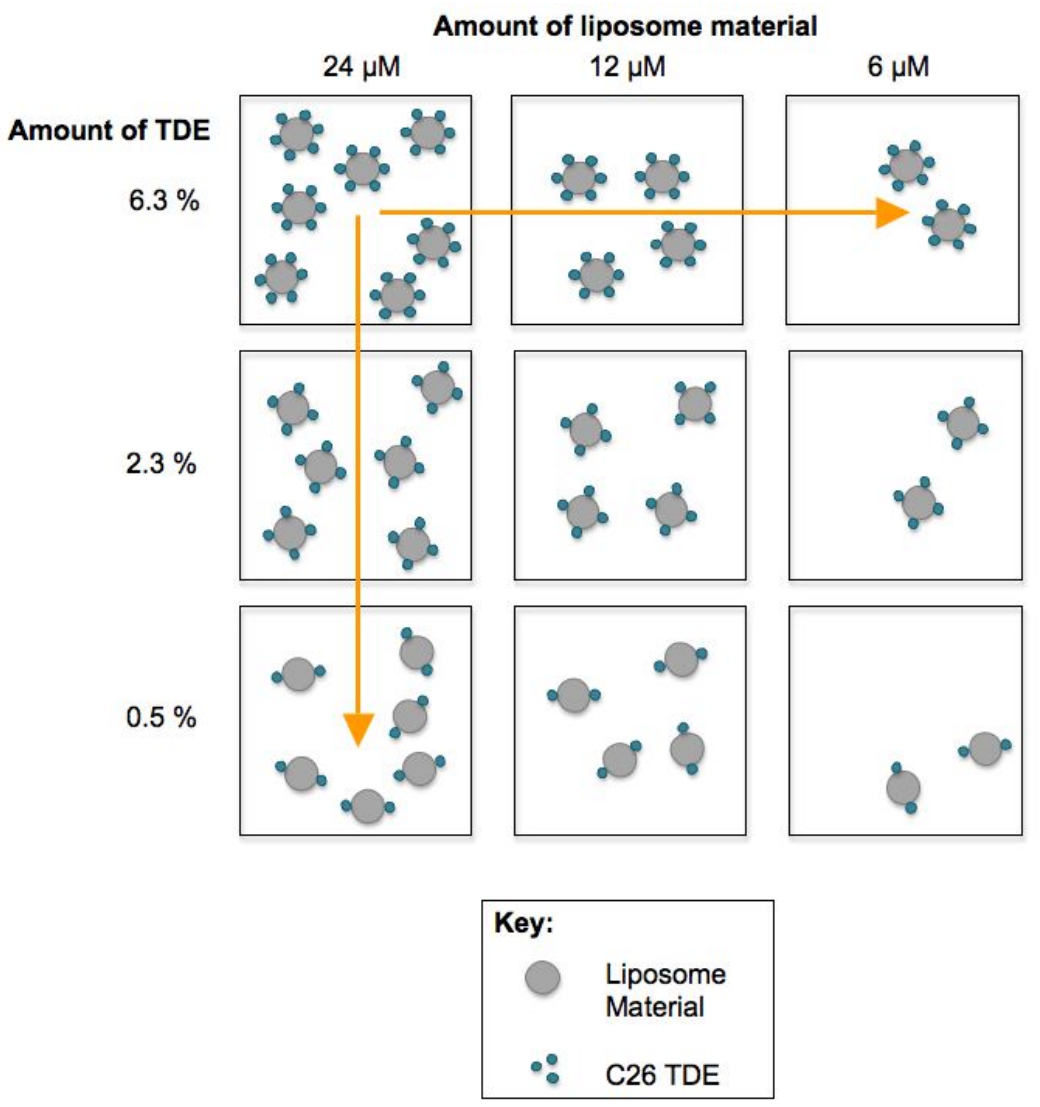

Figure 11. Cartoon representing the three different formulations of C26 TDE incorporated liposomes that were prepared. The lipsomes differed by both the concentration of the liposome solution and by the weight loading of the C26 TDE in the liposome with 6.3, 2.3 and 0.5\% TDE loading.

\subsubsection{The effect of Liposome concentration on NO production by BMMs}

The first experiment performed involved exploring the total amount of C26 TDE that led to an increase in the production of NO by BMMs. To this end, C26 liposomes were formed by adding a $1.0 \mathrm{mg} / \mathrm{mL}$ solution of the C26 TDE when formulating the liposomes. While the target percentage of C26 TDE incorporation was $16 \%$, the actual percentage was determined as $6.3 \%$ of C26 TDE incorporation (see section 3.3 ) into the liposome. The total amount of liposome added to an in vivo culture of BMMs was then serially diluted (Figure 12) and the amount of C26 TDE per suspension was determined 
from the weight percentage loading per liposome stock suspension (full details of these calculations are provided in the experimental section). Thus, the amount of C26 TDE added per experiment (with a C26 TDE loading of 6.3 weight percent) could be determined from the target concentration of the liposomes at $60 \mu \mathrm{M}, 30 \mu \mathrm{M}$ and $15 \mu \mathrm{M}$. Thus, the amount of C26 TDE added into each well, for each of the three experiments correlated to $24 \mu \mathrm{M}, 12 \mu \mathrm{M}$, and $6 \mu \mathrm{M}$, for the respective serial dilutions

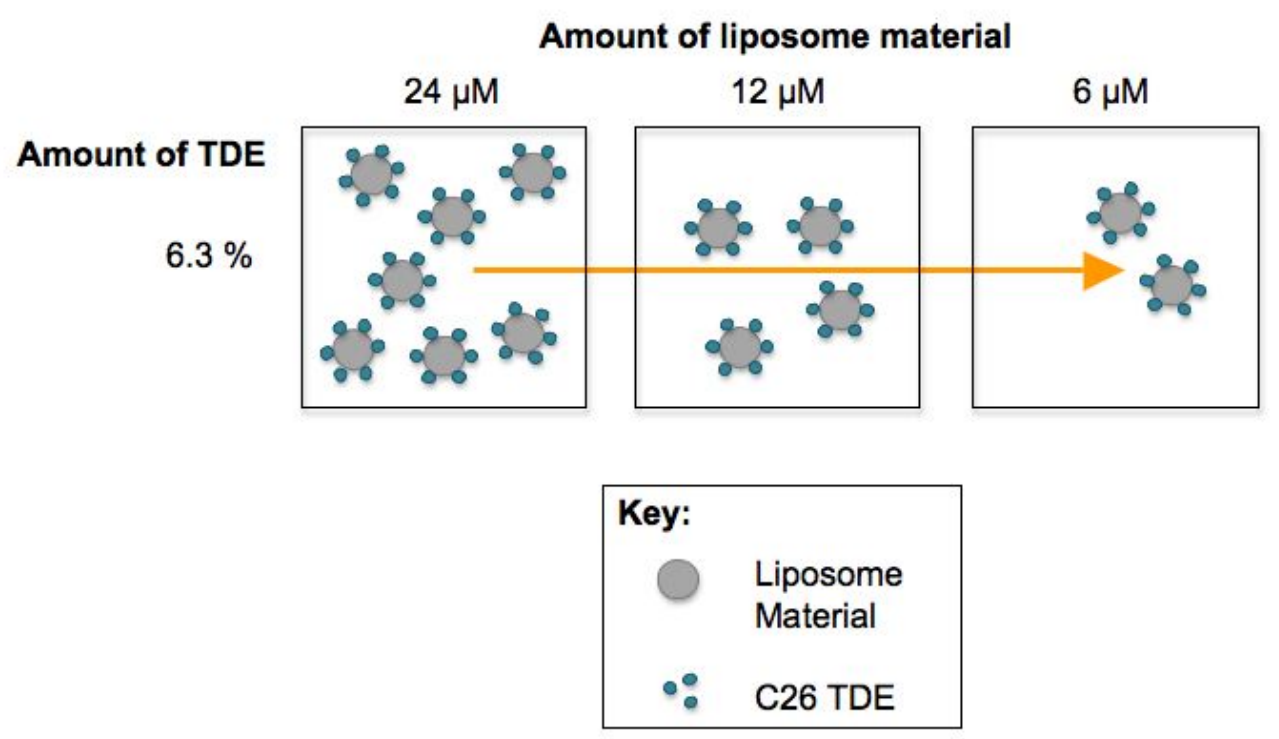

Figure 12. The liposomes containing a $6.3 \%$ weight loading of the C26 TDE for the initial $24 \mu \mathrm{M}$ solution, which was then serially diluted to $12 \mu \mathrm{M}$ and 6 $\mu \mathrm{M}$.

To this end, BMMs cultured from C57BL/6 and Mincle $^{-/-}$mice were thus subjected to C26 TDE incorporated liposomes in successive 2-fold serial dilutions and the Griess assay was conducted on supernatant collected after 24, 48 and 72 h. Again, LPS was used as a positive control, while media only was used as a negative control. As anticipated, there was a concentration dependence of $\mathrm{M} \varphi$ activation in relation to the amount of C26 TDE liposome added in the C57BL/6 mice (Figure 13), with there being negligible activation of BMMs when using liposome concentrations of less than $6 \mu \mathrm{M}$ of 
liposomes with a C26 TDE weight percent loading of 6.3\%. Here, it is also important to note that a general decrease in NO production is observed after $24 \mathrm{~h}$. This observation supports my earlier empty PC/cholesterol liposome studies, whereby the level of NO production reached a maximum at $24 \mathrm{~h}$, after which NO levels drop between the $24-$ $48 \mathrm{hr}$ time period. A slight increase in NO observed at $72 \mathrm{~h}$, again suggests that more non-specific cell death of Mps occurred with longer incubation times with liposomes, (Figure 13).

\section{Dilution of C26 TDE liposomes (6.3 weight \%) in BMMs from C57BL/6 mice}

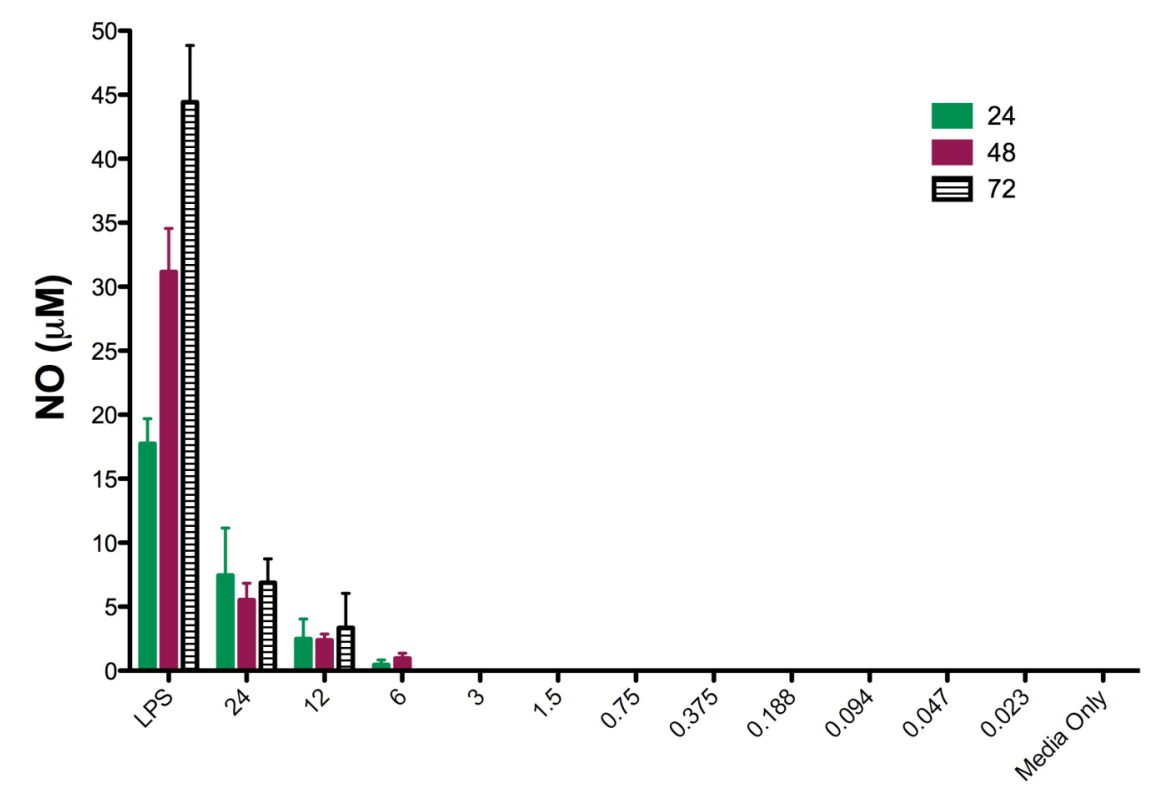

Concentration $(\mu \mathrm{M})$

Figure 13. Titration of $\mathrm{C} 26 \mathrm{TDE}$ liposomes (6.3 weight \%) in C57BL/6 BMMs. NO production by $\mathrm{C} 26 \mathrm{TDE}$ liposome-treated BMMs derived from C57BL/6 mice. BMMs were primed with IFN- $\gamma\left(10 \mathrm{ng} / \mathrm{ml}^{-1}\right)$ and stimulated with a suspension of C26 TDE liposomes at various concentrations of C26 TDE, or with LPS $\left(10 \mathrm{ng} / \mathrm{ml}^{-1}\right)$ or media only. At 24,48 and $72 \mathrm{~h}$, the supernatants were analysed for NO accumulation. For the graph, the means and SDs of triplicate samples from representative experiments performed in duplicate are shown. 
In the case of the Mincle ${ }^{-/-}$BMMs (Figure 14), an unexpected dependence of $\mathrm{M} \varphi$ activation (NO production) with respect to liposome concentration was also observed. Due to the unexpected nature of these results, these experiments were performed three times, with all time points being recorded in triplicate, however, in all experiments, NO was produced by Mincle ${ }^{-/-}$BMMs in response to the liposomes. Again, there was a dependence of NO production by M $\varphi s$ in response to liposome concentrations, with negligible NO being produced at concentrations of less than $6 \mu \mathrm{M}$ of 6.3 weight percent of C26 TDE. What is interesting to note, however, is that in the case of the Mincle ${ }^{-/-}$, NO production peaked at $24 \mathrm{~h}$, with a plateau occurring at $48 \mathrm{~h}$, and then with a subsequent increase in NO production at $72 \mathrm{hr}$, which is probably due to non-specific cell death. ${ }^{127}$ Accordingly, when considered alongside the empty liposome data (Figure 10), the $\mathrm{BMM}_{\mathrm{Mincle}}{ }^{-/-} \mathrm{M} \varphi s$ appear to be a less robust cell line. 


\section{Dilution of C26 TDE liposomes (6.3 weight \%) \\ in BMMs from Mincle $^{-/-}$mice}

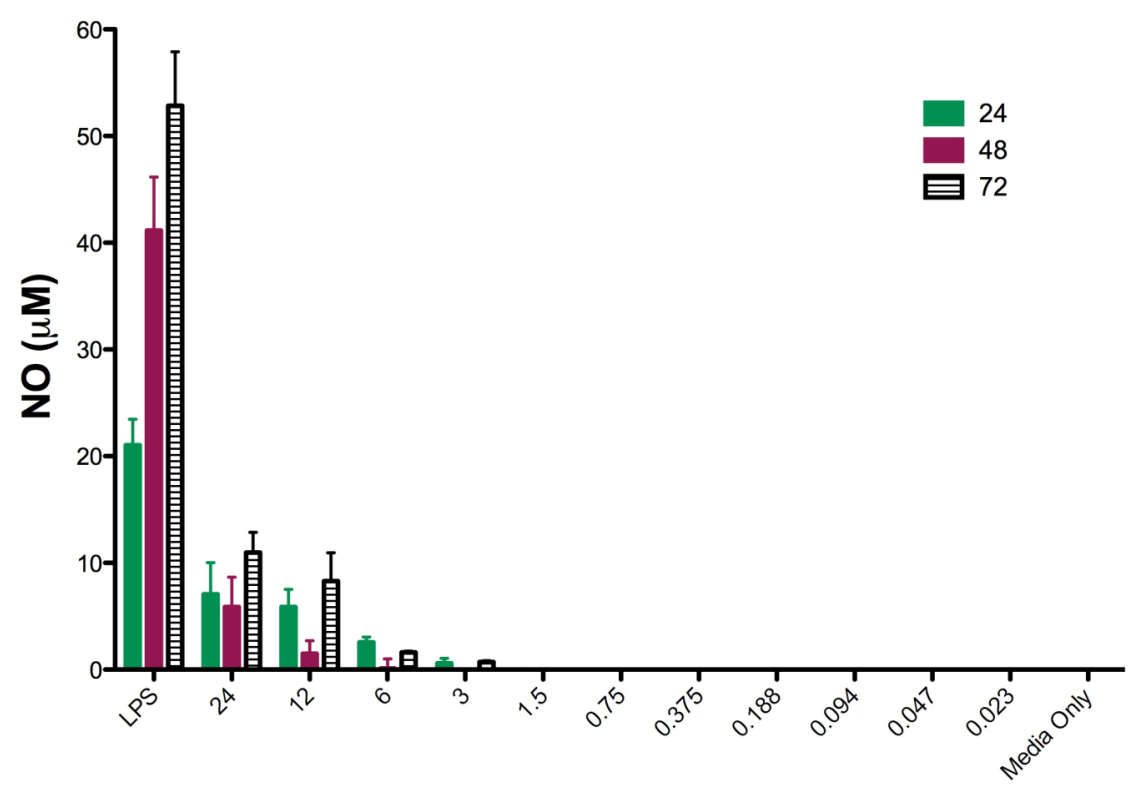

Concentration $(\mu \mathrm{M})$

Figure 14 Titration of C26 TDE liposomes (6.3 weight \%) in Mincle $^{-/-}$BMMs. NO production by C26 TDE liposome-treated BMMs derived from Mincle ${ }^{-/-}$ mice. BMMs were primed with IFN- $\gamma\left(10 \mathrm{ng} / \mathrm{ml}^{-1}\right)$ and stimulated with a suspension of $\mathrm{C} 26 \mathrm{TDE}$ liposomes at various concentrations of $\mathrm{C} 26$ TDE, or with LPS $\left(10 \mathrm{ng} / \mathrm{ml}^{-1}\right)$ or media only. At 24,48 and $72 \mathrm{~h}$, the supernatants were analysed for NO accumulation. For the graph, the means and SDs of triplicate samples from representative experiments performed in duplicate are shown.

Thus, when considering the effect of liposome concentration on $\mathrm{M} \varphi$ activation, it is apparent that more NO is produced with increasing liposome concentration, and that a concentration of liposomes greater than $12 \mu \mathrm{M}$ is required for significant activity. For the reproducibility of responses, it is also suggested that measurements are made at the $48 \mathrm{~h}$ time point or earlier, especially for the $\mathrm{Mincle}^{-/-} \mathrm{M} \varphi$. Initially, the observation that Mincle $^{-/-}$BMMs when treated with C26-TDE liposomes resulted in NO production was unexpected, as it has been previously shown by research in our group ${ }^{118}$ and in studies 
by Lang and co-workers, ${ }^{59}$ that there is no NO production by Mincle $^{-/-}$BMMs in response to the individual TGs. When the glycolipids are incorporated into liposomes, however, it may be possible that the process of $\mathrm{M} \varphi$ phagocytosis activates the BMMs to some extent and that is why NO production is observed by Mincle ${ }^{-/}$, although it should be noted that empty liposomes (containing a $105 \mu \mathrm{M}$ concentration of PC) do not lead to the production of NO (as discussed in section 3.5.1). Since the inception of this project, another receptor for TGs, namely the Macrophage C-type lectin, MCL, was identified and shown to have an influence on the immune response to TGs, ${ }^{63,129}$ and thus, it is also possible that MCL is involved in the phagocytosis of the TG-containing liposomes.

While Mincle is still necessary to generate a robust immune response to TGs and its expression in M $\varphi$ is induced by TGs, MCL is constitutively expressed in myeloid cells and the receptor has been shown to be an endocytic receptor. ${ }^{63}$ Studies involving the cotransfection of non-myeloid cells with MCL, Mincle and FcR $\gamma$, followed by immunoprecipitation, have provided evidence for a covalently linked heterodimer of MCL and Mincle that associated with FcR $\gamma$, which suggests that Mincle acts as a bridge for the interaction between MCL and FcR $\gamma .{ }^{130,131}$ Further, MCL was shown to be required to drive Mincle expression in a Clec4e-GFP fusion reporter mouse. ${ }^{63}$ Moreover, recent work performed in our group has shown that while Mincle is important for the activation of BMMs, the uptake of polystyrene beads coated with TGs is greater than the uptake of non-TG-coated beads, however, the difference in uptake is not Mincle-dependent. ${ }^{132}$ Thus, in light of the results obtained in my experiments, it appears as if Mincle is not the only factor that influences the uptake of liposomes containing TGs. 


\subsubsection{Activity of C26-TDE liposomes compared to C26-TDE solutions}

As the previous experiment showed that C26 TDE liposomes at higher liposome concentration could activate BMMs, next I wanted to investigate how the uptake of C26 TDE liposomes was influenced by different TG-doping levels (at target weight loading percentages of 4,8 and $16 \%$ at $60 \mu \mathrm{M}$, but incorporation as determined by the phenolsulfuric acid assay found actual concentrations to be $24 \mu \mathrm{M}, 17 \mu \mathrm{M}$, and $7.5 \mu \mathrm{M}$ and how NO production by $\mathrm{M} \varphi$ was affected by the delivery mode of the C26 TDE (i.e. as an individual compound or in a liposome formulation). The types of liposomes tested are depicted in Figure 15. 


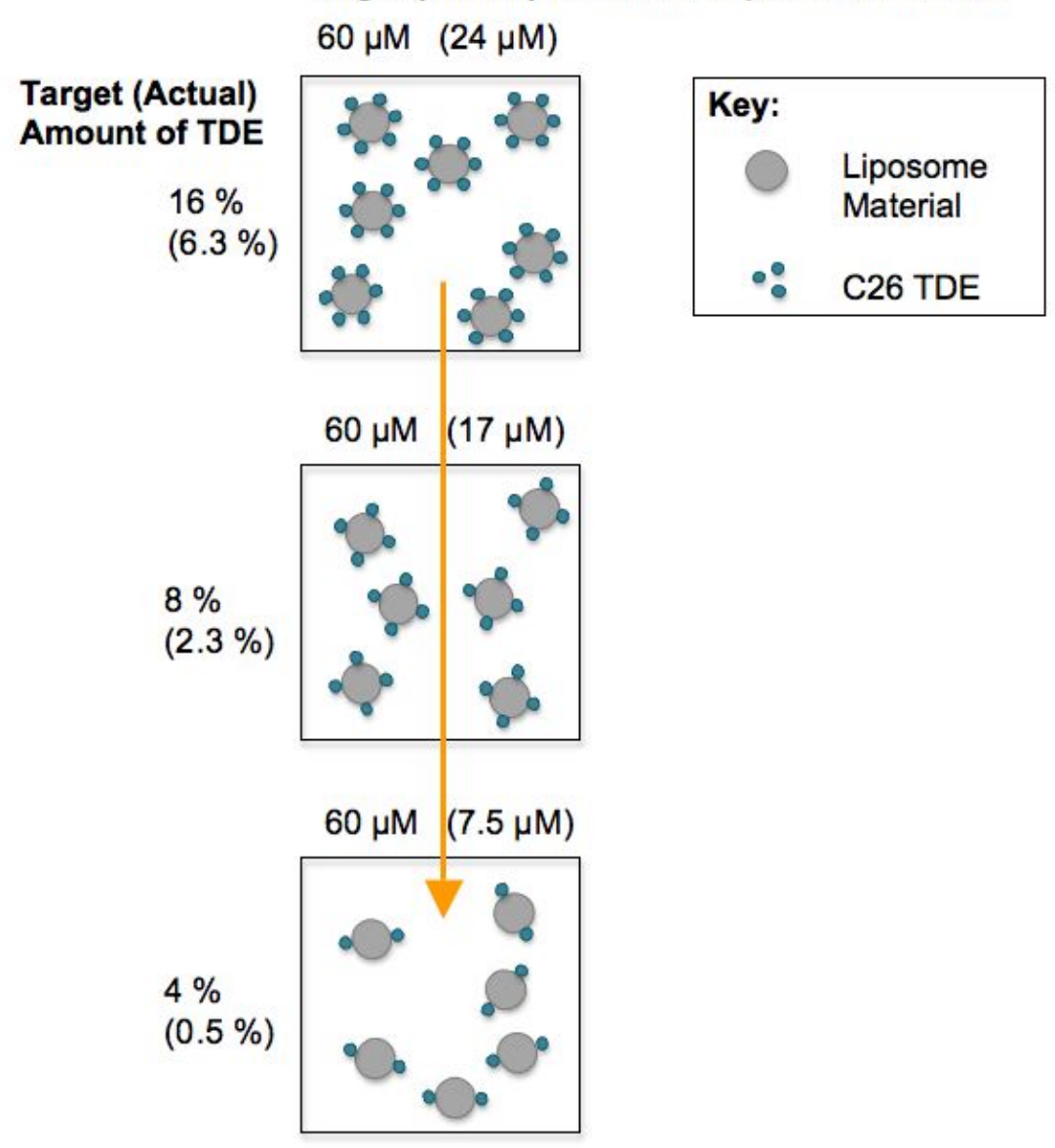

Figure 15. The liposomes containing a weight loading of $6.3,2.3$ and $0.5 \%$ of $\mathrm{C} 26$ TDE

To this end, C26 TDE liposomes or C26 TDE solution (2\% DMSO in PBS) were incubated with BMMs and on day 10, NO production was examined at 24, 48 and $72 \mathrm{~h}$ time points for the liposomes containing different amounts of C26 TDE (Figure 16). As observed, different TDE percentage loadings effect $\mathrm{M} \varphi$ activation to varying degrees when compared to the $\mathrm{C} 26 \mathrm{TDE}$ alone. Liposomes containing 6.3\% C26 TDE show a much greater level of NO production compared to liposomes containing 2.3\% TDE (seen by comparison of the bold blue line compared to the bold green line), however, 
there is less of a difference between NO production by the $2.3 \%$ TG doped liposomes compared the $0.5 \%$ doped liposome. Thus, for an optimal immune response, a doping of at least $6.3 \% \mathrm{C} 26 \mathrm{TDE}$ is required. It also appears that liposomal presentation of C26 TDE leads to a similar level of $\mathrm{M} \varphi$ activation by the TG alone (compare the respective coloured bold and dotted lines). This is a significant finding as less C26 TDE is required to get the same effect, which suggests the addition of TG into the liposome does seem to have some synergistic effect

Thus, in summary, the addition of TG to liposomes appears to increase the activation of $\mathrm{M} \varphi$ to a greater extent than the addition of either the liposomes or the TG alone. While, the measurement of NO production by M $\varphi s$ does not directly provide a measure of the phagocytic capacity of the M $\varphi$, it does suggest that TG-containing liposomes enhance the phagocytic capacity of M $\varphi s$. This hypothesis is further supported by the subsequent studies performed in our group using fluorescent TG-coated beads, ${ }^{132}$ however, from the data gathered to date, it appears as though any such preferential uptake of TG-containing liposomes is not mediated by Mincle. That said, further experiments involving the uptake and flow cytometry analysis of fluorescently labelled liposomes would be required to confirm whether the addition of TGs to liposomes does actually lead to a significant difference in the uptake of liposomes, as only indirect ways to assess this have been performed to date. 


\section{NO Production in BMMs from C57BL/6 mice \\ TDE Liposomes verses C26 TDE Solution}
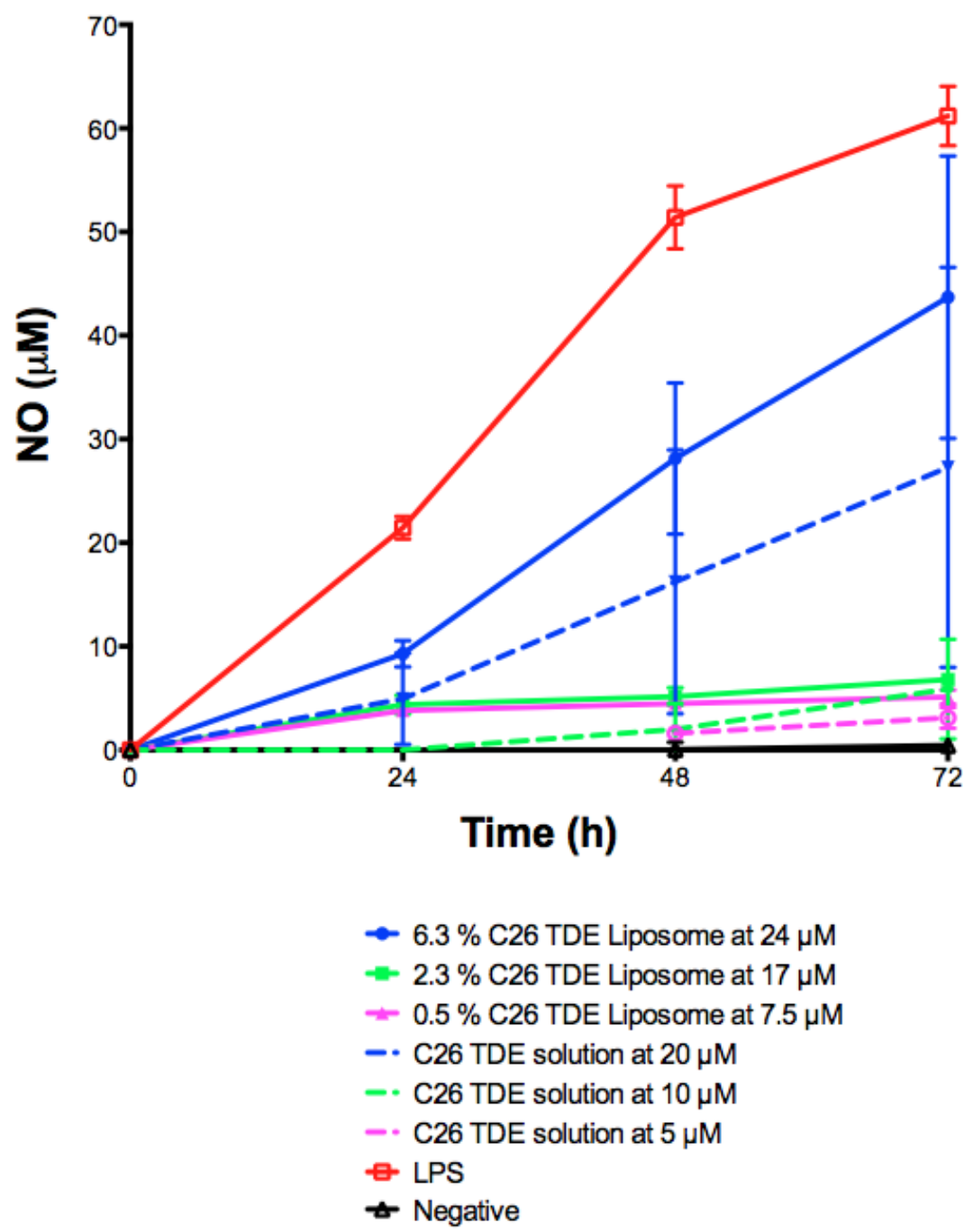

Figure 16. Activation of BMMs from C57BL/6 by C26-TDE liposomes and C26 TDE solutions. BMMs were primed with IFN- $\gamma\left(10 \mathrm{ng} / \mathrm{ml}^{-1}\right)$ and stimulated with a suspension of C26 TDE liposomes alone, C26 solution, or with LPS $\left(10 \mathrm{ng} / \mathrm{ml}^{-1}\right)$ or PBS only $(100 \mu \mathrm{l})$. At 24,48 and $72 \mathrm{~h}$ the supernatants were analysed for NO accumulation. For the graph the means and SDs of triplicate samples from representative experiments performed in duplicate are shown. 


\subsection{Conclusion}

In conclusion, $\mathrm{PC} /$ cholesterol liposomes were found to activate $\mathrm{BMMs}$ derived from $\mathrm{C} 57 \mathrm{BL} / 6$ and Mincle $^{-/-}$mice, as determined by the production of NO. The $\mathrm{PC} /$ Cholesterol liposomes were then titrated from $800 \mu \mathrm{M}$ to $6.25 \mu \mathrm{M}$ to reveal that liposomes containing less than $200 \mu \mathrm{M}$ of PC did not lead to significant NO production by BMMs. A liposome formulation was then developed to contain $105 \mu \mathrm{M}$ of PC, cholesterol, and C26 TDE loading at 0.5, 2.3 and 6.3 weight percentages, with the amount of C26 TDE incorporated into the liposomes being determined by the phenolsulfuric assay. The C26 TDE liposomes are able to activate both C57BL/6 and Mincle ${ }^{-/-}$ BMMs, which suggests that Mincle is not essential for the activation of BMMs. Moreover, there is preferential activation of BMMs by TDE-liposomes at higher concentrations when compared to TDE solutions alone. Taken as a whole, the assay conditions and formulations of the liposomes required for $\mathrm{M} \varphi$ activation have been determined and provisional results have been obtained which suggest that the incorporation of TGs into liposomes does increase the activation of $\mathrm{M} \varphi \mathrm{s}$ (as compared to liposomes or TG alone). These are promising results towards the development of TGliposomes for M $\varphi s-d e p l e t i o n$ therapies for diseases such as cancer. 


\section{CHAPTER 4:}

\section{CONCLUSIONS AND FUTURE PROSPECTS}

\subsection{Conclusions}

The use of bacteria and bacterial components in the treatment of disease is widely documented and is an area of great interest. Accordingly, this thesis sought to explore how TMEs influence the activation of the innate immune response and whether TGs can be used to develop better liposomal drug delivery systems.

In Chapter Two, the synthesis of the C26 TME was explored and an efficient synthetic route for access to these very long chain trehalose monoesters was optimised. As very long chain carboxylic acids have low reactivity, it was found that excess DMAP increased the likelihood of the formation of the activated ester, which then undergoes an intramolecular reaction with an alcohol to give the desired monoester product. It was proposed that excess DMAP reduces the possibility of intermolecular attack from the nitrogen of the activated acid onto the carbonyl of the ester, which would result in the undesired $N$-hexacosanoyl urea side-product. In the biological assessment of the TMEs, it was observed that these monoesters activate $M \varphi s$ in a manner similar to their diester counterparts, whereby the activity of the BMMs was dependent on Mincle. This result is significant as it is the first time that TMEs have been found to activate M $\varphi$ s and demonstrates that only one ester functionality is required for $\mathrm{M} \varphi$ activation.

The synthesis of liposomes containing the C26 TDE was then explored in Chapter Three. Here, the phenol-sulfuric acid assay was used to determine the percentage of TDE incorporated into the liposomes and DLS was used to determine the size range and distribution of both the TG-liposomes and the non-functionalised (PC/cholesterol) 
liposomes. Titration experiments were performed using the PC/cholesterol liposomes, whereby the concentration of PC was decreased from $800 \mu \mathrm{M}$ to $6.25 \mu \mathrm{M}$ in both C57BL/6 and Mincle ${ }^{-/-}$BMMs. PC was found to lead to NO production by M $\varphi s$ at higher concentrations $(\geq 200 \mu \mathrm{M})$, which was thought to be due cell death. Thus, the liposomal formulations for the C26 TDE-containing liposomes were developed so that the solutions contained $105 \mu \mathrm{M}$ of $\mathrm{PC}$ to preserve cell viability and thereby enable the effect of TG incorporation into the liposomes to be more effectively explored. The formulation of C26 TDE incorporated liposomes was explored with weight percentages of loading at $0.5,2.3$ and $6.3 \%$. In this work, the C26 TDE incorporated liposomes were able to activate both the $\mathrm{C} 57 \mathrm{BL} / 6$ and the $\mathrm{Mincle}^{-/-} \mathrm{BMMs}$ in a concentration-dependent manner. It was unusual that the TG-liposomes activated Mincle ${ }^{-/-}$BMMs, however, this may be due to the fact that the BMMs more readily phagocytose the liposomes, or it may be an effect of another more recently identified TG receptor, MCL. The results in this thesis, however, suggest that there is preferential activation of BMMs by TGliposomes compared to individual TGs in solution, though this is dependent on the percentage loading of the TG into the liposome.

Thus, in summary, the research carried out for this thesis contributes towards an understanding of how TG structure influences $M \varphi$ activation. Moreover, the work provides the ground work to suggest that TG-liposomes may be useful tools for more specific M $\varphi s-d e p l e t i o n$ strategies, particularly in diseases such as cancer where TAMs are known to lead to poor patient outcomes. 


\subsection{Future Prospects}

The aforementioned investigations into the biological properties of TGs and the activation of M $\varphi$ s represent significant steps towards the development of adjuvants and drug delivery systems. That said, further studies are required to better explore these respective research areas, particularly with regard to the TG-liposome drug delivery systems.

In particular, Flow Cytometry analysis of the uptake of fluorescent liposomes by M $\varphi s$ is required so that a more direct measure of $M \varphi$ phagocytosis (and hence, the uptake a potential drug inside the liposome) can be better explored. In such studies, a fluorescent dye could be encapsulated within the liposome itself or a fluorescent lipid could be used when synthesising the liposome. Herein, it should be noted that some time was expended on the synthesis of BODIPY (boron-dipyrromethene)-cholesterol in this Master's Thesis, with the particular aim of formulating fluorescent C26-TDE liposomes whereby cholesterol contained a fluorescent reporter group. ${ }^{133}$ Complications, however, arose during the purification of this compound. While some of the desired dye was formed (as determined by ${ }^{1} \mathrm{H}$ and ${ }^{13} \mathrm{C}$ NMR, as well as 2D NMR data), there was insufficient time to optimise the synthetic strategy and silica-gel chromatography proved unsuitable for the separation of the BODIPY starting material from the desired product. Nonetheless, it is envisioned that this strategy could be optimised and that the fluorescent liposomes could then be used in experiments to provide information on liposome uptake and cell activation via the use of Flow Cytometry. Alternatively, another strategy could be explored whereby a dye, such as fluorescein or rhodamine, could be encapsulated inside the liposome so that the fluorescent liposomes could be generated in this manner. ${ }^{134}$

Flow cytometry experiments with the fluorescent liposomes would then allow for more detailed information about the uptake of liposomes by M $\varphi s$. For instance, BMMs incubated with fluorescent PC/cholesterol or fluorescent C26 TDE liposomes can be 
analysed (at various time points) to see if C26 TDE liposomes are preferentially taken up by Mps by measuring Mean Fluorescence Intensity (MFI), as compared to the MFI of liposomes without the TGs. Additionally, the activation of Mps may also be investigated via the detection of specific cell surface markers on the M $\varphi$ s, such as CD80 and CD86, which can be detected by co-staining with fluorescent antibodies and flow analysis. Moreover, in vivo experiments with fluorescent liposomes would allow for investigations into whether specific subtypes of M $\varphi$ s preferentially phagocytose the liposomes. Again, this can be determined by use of Flow Cytometry and the specific $\mathrm{M} \varphi$ sub-populations identified using range of cell surface markers.

Another avenue of future work would be to investigate the effect of MCL on liposome uptake, particularly as MCL is an endocytic receptor. Such studies, however, would require access to $\mathrm{MCL}^{-/-}$mice, which are not currently widely available. It would nonetheless be interesting to ascertain whether MCL is responsible for the activation of M $\varphi$ s by the TG-liposomes. 


\section{CHAPTER 5:}

\section{EXPERIMENTAL PROCEDURES}

General methods and materials for trehalose ester synthesis: Unless stated, all reactions were performed under $\mathrm{N}_{2}$. Prior to use, pyridine was dried and stored over $4 \AA$ molecular sieves (4§ MS), $\mathrm{CH}_{2} \mathrm{Cl}_{2}$ was distilled from $\mathrm{P}_{2} \mathrm{O}_{5}$ and toluene was dried and stored under $\mathrm{Na}$ wire. D-(+)-Trehalose dihydrate (Sigma), anhydrous DMF (Acros), EDCI (Aldrich), DMAP (Merck), hexacosanoic acid (Acros), TBAF (Aldrich), N,Obis(trimethylsilyl)acetamide (Fluka), Dowex- $\mathrm{H}^{+}$(Supelco), anhydrous $\mathrm{Et}_{2} \mathrm{O}$ (Biolab), EtOAc (Pancreac), petroleum ether 40 - 60 (PE) (Pure Science), MeOH (Pancreac), isopropanol (Pure Science), $\mathrm{CHCl}_{3}$ (Pancreac), $\mathrm{NaHCO}_{3}$ (Pure Science), $\mathrm{K}_{2} \mathrm{CO}_{3}$ (Pure Science), $\mathrm{MgSO}_{4}$ (Pure Science) and $\mathrm{NaCl}$ (Pancreac) were used as received. All solvents were removed under reduced pressure. Reactions were monitored by TLC analysis on Macherey-Nagel silica gel coated plastic sheets $(0.20 \mathrm{~mm}$ with fluorescent indicator UV254) via detection by UV absorption (short wave-254 nm; long wave-366 $\mathrm{nm}$ ) and by dipping in $10 \% \mathrm{H}_{2} \mathrm{SO}_{4}$ in $\mathrm{EtOH}$ followed by charring at $150{ }^{\circ} \mathrm{C}$. Column chromatography was performed using Pure Science silica gel (40-63 $\mu \mathrm{m})$. AccuBOND II ODS-C18 (Agilent) was used for reverse chromatography. High resolution mass spectra were recorded on a Waters Q-TOF PremierTM Tandem Mass Spectrometer using positive electro-spray ionisation. Optical rotations were recorded on a PerkinElmer 241 polarimeter or Autopol II (Rudolph Research Analytical) at $589 \mathrm{~nm}$ (sodium D-line). Infrared spectra were recorded as thin films using a Bruker Tensor 27 FTIR spectrometer equipped with an Attenuated Total Reflectance (ATR) sampling accessory and are reported in wave numbers $\left(\mathrm{cm}^{-1}\right)$. Nuclear magnetic resonance spectra were recorded at $20{ }^{\circ} \mathrm{C}$ in $\mathrm{C}_{5} \mathrm{D}_{5} \mathrm{~N}$ or $\mathrm{CDCl}_{3}$ using a Varian INOVA operating at $500 \mathrm{MHz}$. Chemical shifts are given in ppm $(\delta)$. NMR peak assignments were made using COSY, HSQC and HMBC 2D experiments. 


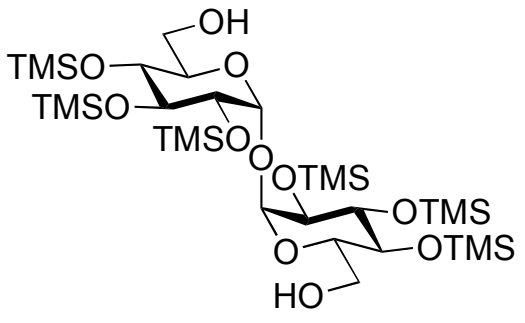

$2,2^{\prime}, 3,3^{\prime}, 4,4^{\prime}$-Hexa- $O$-trimethylsilyl- $\alpha, \alpha^{\prime}$-D-trehalose

(19) Trehalose dihydrate 24 (1.33 g, $3.52 \mathrm{mmol})$ was coevaporated with anhydrous DMF $(3 \times 10 \mathrm{~mL})$ and dissolved in DMF $(5 \quad \mathrm{~mL}) . \quad \mathrm{N}, \mathrm{O}-$ bis(trimethylsilyl)acetamide $(7.4 \mathrm{~mL}, 30.2 \mathrm{mmol})$ and TBAF $(0.04 \mathrm{~mL}, 0.04 \mathrm{mmol})$ were added to this solution, and the mixture was stirred at RT for $1.5 \mathrm{~h}$. The reaction was then quenched by the addition of isopropanol $(1 \mathrm{~mL})$, and the resulting solution was diluted with $\mathrm{MeOH}(80 \mathrm{~mL})$ and cooled to $0{ }^{\circ} \mathrm{C}$. A solution of $\mathrm{K}_{2} \mathrm{CO}_{3}(0.488 \mathrm{~g})$ in $\mathrm{MeOH}(107 \mathrm{~mL})$ was added, and the mixture was stirred at $0{ }^{\circ} \mathrm{C}$ for a further $2 \mathrm{~h}$. The reaction mixture was then neutralised with $\mathrm{AcOH}(0.53$ $\mathrm{mL}$ ) and concentrated in vacuo, and the residue was partitioned between $\mathrm{Et}_{2} \mathrm{O}$ and brine. The aqueous layer was extracted with $\mathrm{Et}_{2} \mathrm{O}(2 \times 50 \mathrm{~mL})$, and the combined organic layers were dried $\left(\mathrm{MgSO}_{4}\right)$, filtered, and concentrated in vacuo. Purification by silica-gel column flash chromatography PE/EtOAc 4:1, v/v) gave diol 19 as a white solid (2.73 g, $3.52 \mathrm{mmol})$ in $94 \%$ yield. $\mathrm{R}_{\mathrm{f}}=0.28(\mathrm{PE} /$ EtOAc $3: 1, \mathrm{v} / \mathrm{v}) ;{ }^{1} \mathrm{H}$ NMR $\left(500 \mathrm{MHz}, \mathrm{CDCl}_{3}\right)$ : $\delta 4.91\left(\mathrm{~d}, J_{1,2}=3.1 \mathrm{~Hz}, 2 \mathrm{H}, \mathrm{H}-1\right), 3.90\left(\mathrm{t}, J_{2,3}=J_{3,4}=9.0 \mathrm{~Hz}, 2 \mathrm{H}, \mathrm{H}-3\right), 3.88-3.84(\mathrm{~m}$, $2 \mathrm{H}, \mathrm{H}-5), 3.72\left(\mathrm{dd}, J_{6 \mathrm{a}, 6 \mathrm{~b}}=11.8, J_{5,6 \mathrm{a}}=2.7 \mathrm{~Hz}, 2 \mathrm{H}, \mathrm{H}-6 \mathrm{a}\right), 3.70,\left(\mathrm{dd}, J_{6 \mathrm{a}, 6 \mathrm{~b}}=11.8, J_{5,6 \mathrm{~b}}=\right.$ $3.9 \mathrm{~Hz}, 2 \mathrm{H}, \mathrm{H}-6 \mathrm{~b}), 3.49$ (t, $\left.J_{3,4}=J_{4,5}=9.0 \mathrm{~Hz}, 2 \mathrm{H}, \mathrm{H}-4\right), 3.42$ (dd, $J_{2,3}=9.0, J_{1,2}=3.1$ $\mathrm{Hz}, 2 \mathrm{H}, \mathrm{H}-2), 0.17,0.15,0.13 \mathrm{ppm}\left(3 \times \mathrm{s}, 54 \mathrm{H}, \mathrm{CH}_{3}, \mathrm{TMS}\right)$; All other spectroscopic data and analyses were repeated and matched those reported by Khan et al. ${ }^{28}$

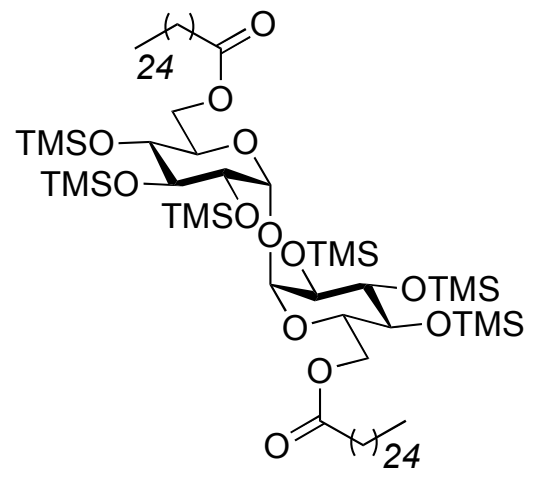

6,6'-Di- $O$-hexacosanoyl-2,2',3,3',4,4'-hexa- $O$ trimethylsilyl-a, $\boldsymbol{\alpha}^{\prime}$-D-trehalose (25) EDCI $(0.73 \mathrm{~g}$, $4.69 \mathrm{mmol})$ and DMAP (0.026 g, $2.13 \mathrm{mmol})$ were added to a solution of diol 19 (1.102 g, $1.42 \mathrm{mmol})$ and hexacosanoic acid (37.9 $\mathrm{mg}, 0.096 \mathrm{mmol})$ in dry toluene $(5 \mathrm{~mL})$. The mixture was stirred at $70{ }^{\circ} \mathrm{C}$ until TLC showed the reaction to be complete (6 days). The resulting precipitate was removed by filtration and washed with EtOAc $(2 \times 50 \mathrm{~mL})$; the 
combined organic layers were washed with water $(50 \mathrm{~mL})$ and brine $(40 \mathrm{~mL})$, dried $\left(\mathrm{MgSO}_{4}\right)$, filtered and concentrated in vacuo. The product was purified by silica-gel flash column chromatography (PE/EtOAc 50:1, v/v) to give TDE 25 as a white solid (2.16 g, $1.42 \mathrm{mmol}, 85 \%) . \mathrm{R}_{\mathrm{f}}=0.84(\mathrm{PE} / \mathrm{EA}, 5 / 1, \mathrm{v} / \mathrm{v}) ;{ }^{1} \mathrm{H} \mathrm{NMR}\left(500 \mathrm{MHz}, \mathrm{CDCl}_{3}\right) \delta$ $4.92\left(\mathrm{~d}, J_{1,2}=2.9 \mathrm{~Hz}, 2 \mathrm{H}, \mathrm{H}-1\right), 4.28\left(\mathrm{dd}, J_{6 \mathrm{a}, 6 \mathrm{~b}}=11.7 \mathrm{~Hz}, J_{5,6 \mathrm{a}}=2.2 \mathrm{~Hz}, 2 \mathrm{H}, \mathrm{H}-6 \mathrm{a}\right), 4.06$ $\left(\mathrm{dd}, J_{6 \mathrm{a}, 6 \mathrm{~b}}=11.7 \mathrm{~Hz}, J_{5,6 \mathrm{~b}}=4.7 \mathrm{~Hz}, 2 \mathrm{H}, \mathrm{H}-6 \mathrm{~b}\right), 4.02-3.98(\mathrm{~m}, 2 \mathrm{H}, \mathrm{H}-5), 3.91$ (t, $J_{2,3}=$ $\left.J_{3,4}=9.0 \mathrm{~Hz}, 2 \mathrm{H}, \mathrm{H}-3\right), 3.48\left(\mathrm{t}, J_{3,4}=J_{4,5}=9.0 \mathrm{~Hz}, 2 \mathrm{H}, \mathrm{H}-4\right), 3.45$ (dd, $J_{2,3}=9.0 \mathrm{~Hz}, J_{1,2}$ $=2.9 \mathrm{~Hz}, 2 \mathrm{H}, \mathrm{H}-2), 2.36\left(\mathrm{dt}, J_{8 \mathrm{a}, 8 \mathrm{~b}}=13.6 \mathrm{~Hz}, J_{8 \mathrm{a}, 9}=7.3 \mathrm{~Hz}, 2 \mathrm{H}, \mathrm{H}-8 \mathrm{a}\right), 2.34\left(\mathrm{dt}, J_{8 \mathrm{a}, 8 \mathrm{~b}}=\right.$ $13.6 \mathrm{~Hz}, J_{8 \mathrm{~b}, 9}=7.0 \mathrm{~Hz}, 2 \mathrm{H}, \mathrm{H}-8 \mathrm{~b}$ ), 1.64-1.60 (m, 4H, H-9), 1.34-1.18 (m, 88H, H-10$\mathrm{H}-31), 0.89$ (t, $\left.J_{31,32}=6.7 \mathrm{~Hz}, \mathrm{H}-32\right), 0.16,0.14,0.137 \mathrm{ppm}\left(3 \times_{\mathrm{s}}, 54 \mathrm{H}, \mathrm{CH} 3, \mathrm{TMS}\right) ;$ All other spectroscopic data and analyses were repeated and matched those reported by Khan et al. ${ }^{28}$

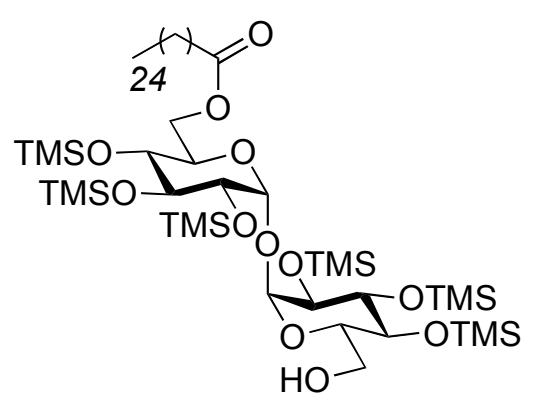

\section{$2,2^{\prime}, 3,3^{\prime}, 4,4^{\prime}$-Hexa-O-trimethylsilyl-6-hexacosanoyl-} 6'-hydroxy-a, $\boldsymbol{\alpha}^{\prime}$-D-trehalose (31) EDCI (0.20 mmol, 2 equiv) and DMAP (0.44 mmol, 4.4 equiv) were added to a solution of $2,2^{\prime}, 3,3^{\prime}, 4,4^{\prime}$-hexa- $O$ trimethylsilyl- $\alpha, \alpha^{\prime}$-D-trehalose (19, 0.1 mmol, 1 equiv.) and hexacosanoic acid ( $0.18 \mathrm{mmol}, 1.8$ equiv) in dry toluene $(5 \mathrm{~mL})$. The reaction mixture was stirred at $70{ }^{\circ} \mathrm{C}$ for seven days. The resulting precipitate was removed by filtration, and the precipitate was washed thoroughly with EtOAc $(2 \times 50 \mathrm{~mL})$. The combined organic layers were washed with water $(50 \mathrm{~mL})$ and brine $(40 \mathrm{~mL})$, dried $\left(\mathrm{MgSO}_{4}\right)$, filtered and concentrated in vacuo. The product was purified by silica gel flash chromatography (elution with Pet.Ether/ EtOAc 20:1) to give TME 33 in 45\% yield. $\mathrm{R}_{\mathrm{f}}=0.62(\mathrm{PE} / \mathrm{EA} 5: 1, \mathrm{v} / \mathrm{v}) ;{ }^{1} \mathrm{H}$ NMR (500 MHz, $\mathrm{CDCl}_{3}$ ): $\delta 4.93$ $\left(\mathrm{d}, J_{1 / 1^{\prime}, 2 / 2^{\prime}}=3.5 \mathrm{~Hz}, 1 \mathrm{H}, \mathrm{H}-1 / 1^{\prime}\right), 4.92\left(\mathrm{~d}, J_{1 / 1^{\prime}, 2 / 2^{\prime}}=3.5 \mathrm{~Hz}, 1 \mathrm{H}, \mathrm{H}-1 / 1^{\prime}\right), 4.30\left(\mathrm{dd}, J_{6 \mathrm{a}, 6 \mathrm{~b}}=\right.$ $\left.11.7 \mathrm{~Hz}, J_{5,6 \mathrm{a}}=2.2 \mathrm{~Hz}, 1 \mathrm{H}, \mathrm{H}-6 \mathrm{a}\right), 4.07\left(\mathrm{dd}, J_{6 \mathrm{a}, 6 \mathrm{~b}}=11.7 \mathrm{~Hz}, J_{5,6 \mathrm{~b}}=4.7 \mathrm{~Hz}, 1 \mathrm{H}, \mathrm{H}-6 \mathrm{~b}\right)$, 4.03-3.99 (m, 1H, H-5), $3.91\left(\mathrm{t}, J_{2 / 2^{\prime}, 3 / 3^{\prime}}=J_{3 / 3^{\prime}, 4 / 4^{\prime}}=9.0 \mathrm{~Hz}, 1 \mathrm{H}, \mathrm{H}-3 / 3^{\prime}\right), 3.90\left(\mathrm{t}, J_{2 / 2^{\prime}, 3 / 3^{\prime}}=\right.$ $\left.J_{3 / 3^{\prime}, 4 / 4}=9.0 \mathrm{~Hz}, 1 \mathrm{H}, \mathrm{H}-3 / 3^{\prime}\right), 3.85\left(\mathrm{dt}, J_{4^{\prime}, 5^{\prime}}=9.6 \mathrm{~Hz}, J_{5^{\prime}, 6 \mathrm{a}^{\prime}}=J_{5^{\prime}, 6 \mathrm{~b}^{\prime}}=3.4 \mathrm{~Hz}, 1 \mathrm{H}, \mathrm{H}-5^{\prime}\right)$, 
$3.71\left(\mathrm{dd}, J_{6 \mathrm{a}^{\prime}, 6 \mathrm{~b}^{\prime}}=11.7 \mathrm{~Hz}, J_{5^{\prime}, 6 \mathrm{a}^{\prime}}=3.0 \mathrm{~Hz}, 1 \mathrm{H}, \mathrm{H}-6 \mathrm{a}^{\prime}\right), 3.69\left(\mathrm{dd}, J_{6 \mathrm{a}^{\prime}, 6 \mathrm{~b}^{\prime}}=11.7 \mathrm{~Hz}, J_{5^{\prime}, 6 \mathrm{~b}^{\prime}}=\right.$ $\left.3.9 \mathrm{~Hz}, 1 \mathrm{H} ; \mathrm{H}-6 \mathrm{~b}^{\prime}\right), 3.49$ (t, $\left.J_{3,4}=J_{4,5}=9.0 \mathrm{~Hz}, 1 \mathrm{H}, \mathrm{H}-4\right), 3.48\left(\mathrm{t}, J_{3^{\prime}, 4^{\prime}}=J_{4^{\prime}, 5^{\prime}}=9.0 \mathrm{~Hz}, 1 \mathrm{H}\right.$, H-4'), $3.44\left(\mathrm{dd}, J_{2,3 / 2^{\prime}, 3^{\prime}}=9.0 \mathrm{~Hz}, J_{1,2 / 1^{\prime}, 2^{\prime}}=3.5 \mathrm{~Hz}, 1 \mathrm{H}, \mathrm{H}-2 / 2^{\prime}\right), 3.43\left(\mathrm{dd}, J_{2,3 / 2^{\prime}, 3^{\prime}}=9.0 \mathrm{~Hz}\right.$, $\left.J_{1,2 / 1^{\prime}, 2^{\prime}}=3.5 \mathrm{~Hz}, 1 \mathrm{H}, \mathrm{H}-2 / 2^{\prime}\right), 2.36\left(\mathrm{dt}, J_{8 \mathrm{a}, 8 \mathrm{~b}}=12.5 \mathrm{~Hz}, J_{8 \mathrm{a}, 9}=7.1 \mathrm{~Hz}, 1 \mathrm{H} ; \mathrm{H}-8 \mathrm{a}\right), 2.34$ $\left(\mathrm{dt}, J_{8 \mathrm{a}, 8 \mathrm{~b}}=12.5, J_{8 \mathrm{~b}, 9}=7.0 \mathrm{~Hz}, 1 \mathrm{H}, \mathrm{H}-8 \mathrm{~b}\right), 1.63$ (pent, $J_{\alpha, \beta}=J_{\beta, \gamma}=6.8 \mathrm{~Hz}, 2 \mathrm{H}, \mathrm{H}-9$ ), 1.38-1.20 (m, 44H, H-10 to H-31), 0.89 (t, $J_{31,32}=6.8$ Hz, 3H, H-32), 0.17, 0.16, 0.15, $0.14,0.13 \mathrm{ppm}\left(5 \times \mathrm{s}, 54 \mathrm{H}, \mathrm{CH}_{3}, \mathrm{TMS}\right)$. All other spectroscopic data and analyses were repeated and matched those reported by Stocker et al. ${ }^{118}$

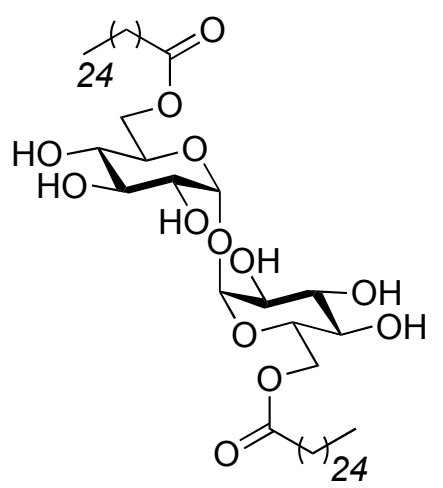

6,6'-Di- $O$-hexacosanoyl- $\alpha, \alpha^{\prime}$-D-trehalose (27) Dowex- $\mathrm{H}^{+}$ (3.0 mg) was added to a solution of TDE 25 (30.0 mg, 0.02 mmol) in $\mathrm{CH}_{2} \mathrm{Cl}_{2} / \mathrm{MeOH}(5 \mathrm{~mL}, 1: 1, \mathrm{v} / \mathrm{v})$, and the reaction mixture was stirred at room temperature. After $30 \mathrm{~min}$, the mixture was filtered and concentrated in vacuo, and the resulting residue was subjected to reversed-phase ODS-C18 column chromatography (eluting in pyridine) to obtain the fully deprotected compound 27 as a white solid (11.18 $\mathrm{mg}, 0.010 \mathrm{mmol}$ ) in 65\% yield. $\mathrm{R}_{\mathrm{f}}=0.52(\mathrm{EA} / \mathrm{MeOH}, 95 / 5, \mathrm{v} / \mathrm{v}) ;{ }^{1} \mathrm{H} \mathrm{NMR}\left(500 \mathrm{MHz}, \mathrm{C}_{5} \mathrm{H}_{5} \mathrm{~N}\right) \delta 5.78\left(\mathrm{~d}, J_{1,2}=3.7 \mathrm{~Hz}\right.$, $2 \mathrm{H}, \mathrm{H}-1), 4.98-4.96(\mathrm{~m}, 2 \mathrm{H}, \mathrm{H}-5), 4.87(\mathrm{~m}, 2 \mathrm{H}, \mathrm{H}-6 \mathrm{a}), 4.72\left(\mathrm{dd}, J_{6 \mathrm{a}, 6 \mathrm{~b}}=11.7 \mathrm{~Hz}, J_{5,6 \mathrm{a}}=\right.$ $5.4 \mathrm{~Hz}, 2 \mathrm{H}, \mathrm{H}-6 \mathrm{~b}), 4.64\left(\mathrm{t}, J_{2,3}=J_{3,4}=9.0 \mathrm{~Hz}, 2 \mathrm{H}, \mathrm{H}-3\right), 4.21\left(\mathrm{dd}, J_{2,3}=9.0 \mathrm{~Hz}, J_{1,2}=\right.$ $3.7 \mathrm{~Hz}, 2 \mathrm{H}, \mathrm{H}-2), 4.01\left(\mathrm{t}, J_{3,4}=J_{4,5}=9.0 \mathrm{~Hz}, 2 \mathrm{H}, \mathrm{H}-4\right), 2.23\left(\mathrm{dt}, J_{8 \mathrm{a}, 8 \mathrm{~b}}=12.2 \mathrm{~Hz}, J_{8 \mathrm{a}, 9}=\right.$ $7.5 \mathrm{~Hz}, 2 \mathrm{H}, \mathrm{H}-8 \mathrm{a}), 2.17\left(\mathrm{dt}, J_{8 \mathrm{a}, 8 \mathrm{~b}}=12.2 \mathrm{~Hz}, J_{8 \mathrm{a}, 9}=7.5 \mathrm{~Hz}, 2 \mathrm{H}, \mathrm{H}-8 \mathrm{~b}\right.$ ), 1.50 (pent, $J_{\alpha, \beta}=$ $\left.J_{\beta, \gamma}=7.4 \mathrm{~Hz}, 4 \mathrm{H}, \mathrm{H}-9\right), 1.26-1.04(\mathrm{~m}, 88 \mathrm{H}, \mathrm{H}-10-\mathrm{H}-31), 0.73 \mathrm{ppm}\left(\mathrm{t}, J_{31,32}=6.8 \mathrm{~Hz}\right.$, $6 \mathrm{H}, \mathrm{H}-32)$; All other spectroscopic data and analyses were repeated and matched those reported by Khan et al. ${ }^{28}$ 


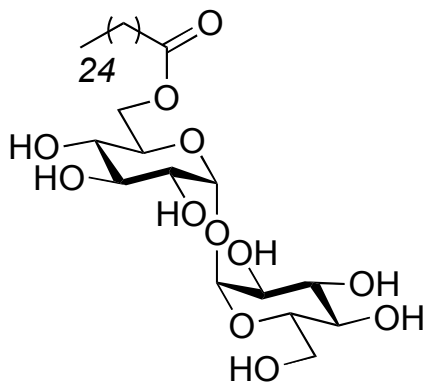

6-O-Hexacosanoyl- $\boldsymbol{\alpha}, \boldsymbol{\alpha}^{\prime}$-D-trehalose (35): Dowex- $\mathrm{H}^{+}(0.5$ $\mathrm{mg}$ ) was added to a solution of TME $33(5 \mathrm{mg}, 0.004 \mathrm{mmol})$ in $\mathrm{CH}_{2} \mathrm{Cl}_{2} / \mathrm{MeOH}(0.5 \mathrm{~mL}, 1: 1, \mathrm{v} / \mathrm{v})$, and the reaction mixture was stirred at room temperature. After $30 \mathrm{~min}$, the mixture was filtered and concentrated in vacuo, and the resulting residue was subjected to reversed-phase column chromatography (elution with $2 \% \mathrm{MeOH}$ in $\mathrm{H}_{2} \mathrm{O}$ ) to obtain the fully deprotected compound 35 as a white solid (3 mg, 0.004) in $97 \%$ yield. $\mathrm{R}_{\mathrm{f}}=0.38(5 \% \mathrm{MeOH}$ in EtOAc); ${ }^{1} \mathrm{H}$ NMR $\left(500 \mathrm{MHz}, \mathrm{C}_{5} \mathrm{H}_{5} \mathrm{~N}\right): \delta 5.84\left(\mathrm{~d}, J_{1 / 1^{\prime}, 2 / 2^{\prime}}=3.9 \mathrm{~Hz}, 1 \mathrm{H}, \mathrm{H}-1 / 1^{\prime}\right), 5.82(\mathrm{~d}$, $\left.J_{1 / 1^{\prime}, 2 / 2^{\prime}}=3.9 \mathrm{~Hz}, 1 \mathrm{H}, \mathrm{H}-1 / 1^{\prime}\right), 5.01-5.91(\mathrm{~m}, 1 \mathrm{H}, \mathrm{H}-5), 4.89\left(\mathrm{dd}, J_{6 \mathrm{a}, 6 \mathrm{~b}}=11.5 \mathrm{~Hz}, J_{5,6 \mathrm{a}}=\right.$ $1.9 \mathrm{~Hz}, 1 \mathrm{H}, \mathrm{H}-6 \mathrm{a}), 4.89-4.86\left(\mathrm{~m}, 1 \mathrm{H}, \mathrm{H}-5^{\prime}\right), 4.73\left(\mathrm{dd}, J_{6 \mathrm{a}, 6 \mathrm{~b}}=11.5 \mathrm{~Hz}, J_{5,6 \mathrm{~b}}=5.2 \mathrm{~Hz}, 1 \mathrm{H}\right.$, $\mathrm{H}-6 \mathrm{~b}$ ), $4.70\left(\mathrm{t}, J_{2 / 2^{\prime}, 3 / 3^{\prime}}=J_{3 / 3^{\prime}, 4 / 4^{\prime}}=8.9 \mathrm{~Hz}, 1 \mathrm{H}, \mathrm{H}-3 / 3^{\prime}\right), 4.68\left(\mathrm{t}, J_{2 / 2^{\prime}, 3 / 3^{\prime}}=J_{3 / 3^{\prime}, 4 / 4^{\prime}}=8.9 \mathrm{~Hz}\right.$, $\left.1 \mathrm{H}, \mathrm{H}-3 / 3^{\prime}\right), 4.37\left(\mathrm{dd}, J_{6 \mathrm{a}^{\prime}, 6 \mathrm{~b}^{\prime}}=11.7 \mathrm{~Hz}, J_{5^{\prime}, 6 \mathrm{a}^{\prime}}=2.5 \mathrm{~Hz}, 1 \mathrm{H}, \mathrm{H}-6 \mathrm{a}^{\prime}\right), 4.31\left(\mathrm{dd}, J_{6 \mathrm{a}^{\prime}, 6 \mathrm{~b}^{\prime}}=11.7\right.$ $\left.\mathrm{Hz}, J_{5^{\prime}, 6 \mathrm{a}^{\prime}}=4.9 \mathrm{~Hz}, 1 \mathrm{H}, \mathrm{H}-6 \mathrm{~b}^{\prime}\right), 4.22\left(\mathrm{dd}, J_{2,3 / 2^{\prime}, 3^{\prime}}=9.4 \mathrm{~Hz}, J_{1,2 / 1^{\prime}, 2^{\prime}}=3.9 \mathrm{~Hz}, 1 \mathrm{H}, \mathrm{H}-2 / 2^{\prime}\right)$, $4.19\left(\mathrm{dd}, J_{3^{\prime}, 4^{\prime}}=J_{4^{\prime}, 5^{\prime}}=9.4 \mathrm{~Hz}, 1 \mathrm{H}, \mathrm{H}-4^{\prime}\right), 4.18\left(\mathrm{dd}, J_{2,3 / 2^{\prime}, 3^{\prime}}=9.4 \mathrm{~Hz}, J_{1,2 / 1^{\prime}, 2^{\prime}}=3.9 \mathrm{~Hz}, 1 \mathrm{H}\right.$, $\left.\mathrm{H}-2 / 2^{\prime}\right), 4.08\left(\mathrm{t}, J_{3^{\prime}, 4^{\prime}}=J_{4^{\prime}, 5^{\prime}}=9.4 \mathrm{~Hz}, 1 \mathrm{H}, \mathrm{H}-4\right), 2.22\left(\mathrm{dt}, J_{8 \mathrm{a}, 8 \mathrm{~b}}=11.5 \mathrm{~Hz}, J_{8 \mathrm{a}, 9}=7.4 \mathrm{~Hz}\right.$, $1 \mathrm{H}, \mathrm{H}-8 \mathrm{a}), 2.21\left(\mathrm{dd}, J_{8 \mathrm{a}, 8 \mathrm{~b}}=11.5 \mathrm{~Hz}, J_{8 \mathrm{~b}, 9}=7.4 \mathrm{~Hz}, 1 \mathrm{H}, \mathrm{H}-8 \mathrm{~b}\right), 1.51$ (pent, $J_{\alpha, \beta}=J_{\beta, \gamma}=$ $7.3 \mathrm{~Hz}, 2 \mathrm{H}, \mathrm{H}-9$ ), $1.27-1.06$ (m, 36H, H-10 to H-31), $0.74 \mathrm{ppm}$ (t, $J_{31,32}=7.1 \mathrm{~Hz}, 3 \mathrm{H}$, H-32). All other spectroscopic data and analyses were repeated and matched those reported by Stocker et al. ${ }^{118}$

The TBD and C22 TME were prepared as a side product by Dr. Ashna Khan. 
General methods and materials for Liposome synthesis: The lipids, L- $\alpha-$ phosphatidylcholine and cholesterol were purchased from Sigma Aldrich and BDH respectively. Chloroform was supplied by Pancreac and Phosphate Buffered Saline (PBS) by Invitrogen. Stock solutions of phosphatidylcholine $(1 \mathrm{mg} / \mathrm{mL})$ and cholesterol $(1 \mathrm{mg} / \mathrm{mL})$ in chloroform were prepared in advance and stored at $-20{ }^{\circ} \mathrm{C}$ under Argon. Argon is used to prevent oxidation of phosphatidylcholine. A stock solution of C26 TDE $(1 \mathrm{mg} / \mathrm{mL})$ was also prepared in $\mathrm{CHCl}_{3} / \mathrm{EtOH}(1: 1, \mathrm{v} / \mathrm{v})$ and stored under Argon.

The preparation of the liposomes was performed in a similar way as previously described by van Rooijen et al. via the dried-lipid film hydration method, ${ }^{96}$ followed by sonication. Here, cholesterol and phosphatidylcholine (5.3:1 molar ratio) were dissolved in cholorform. A dried lipid film was obtained by evaporation of organic solvents under reduced pressure at approximately $45{ }^{\circ} \mathrm{C}$ for 30 minutes. The lipid layer was dispersed with phosphate buffer solution (PBS) by gentle rotation for $2 \mathrm{~h}$ at RT. The resulting suspension was then gently shaken and sonicated in a waterbath at $40 \mathrm{~Hz}$ (Ultrasonic cleaner FXP8M) for $3 \mathrm{~min}$. The liposome suspension was held under argon gas overnight at $4{ }^{\circ} \mathrm{C}$ and used within 3 days. A representative experiment for each different liposome formulation is given below.

Procedure for empty PC/cholesterol liposomes: $8.07 \mathrm{~mL}(8.07 \mathrm{mg}, 10.3 \mu \mathrm{mol})$ of phosphatidylcholine stock solution was added to $0.75 \mathrm{~mL}(0.75 \mathrm{mg}, 1.94 \mu \mathrm{mol})$ of cholesterol stock solution in a $500 \mathrm{~mL}$ round bottom flask. The chloroform was removed by low vacuum (minimum 120 mbar) evaporation, to give a white phospholipid film that formed against the inside of the flask. The lipid film was then dispersed in $3 \mathrm{~mL}$ PBS by gentle rotation (maximum $180 \mathrm{rpm}$ ) at RT for $30 \mathrm{~min}$. The resulting milky white suspension was then held under nitrogen gas at RT for $2 \mathrm{~h}$, then shaken gently and sonicated in a water bath for $3 \mathrm{~min}$. This suspension was then stored under nitrogen gas at $\mathrm{RT}$ overnight at $4{ }^{\circ} \mathrm{C}$ to allow for the swelling of the liposomes. The suspension is gently shaken before administration to cells or before diluting, in order to achieve a homogeneous distribution of the liposomes in suspension. 
General Procedure for TDE containing liposomes: The preparation of TDE containing liposomes follows the procedure of empty PC/cholesterol liposomes, as described above. Additionally, a known quantity (see below) of TDE was dissolved in $\mathrm{CHCl}_{3} / \mathrm{EtOH}(1: 1, \mathrm{v} / \mathrm{v})$, and added to the mixture of PC and cholesterol in chloroform, before evaporation of organic solvents under reduced pressure to give a dried lipid film. The resulting lipid film was then re-dissolved in $\mathrm{CHCl}_{3}$ and co-evaporated twice with $\mathrm{CHCl}_{3}$ to ensure complete removal of EtOH. Dispersion of the lipid film was then achieved, as previously described.

For 3.4.2.2: $\quad 4 \%$ weight loading of C26 TDE in liposome (actual: 2.5\%):

C26 TDE $(0.537 \mathrm{mg}, 0.488 \mu \mathrm{mol})$ dissolved in $1: 1 \mathrm{CHCl}_{3} / \mathrm{EtOH}$ was added to the mixture of PC $(8.07 \mathrm{mg}, 10.3 \mu \mathrm{mol})$ and cholesterol $(0.75 \mathrm{mg}, 1.94 \mu \mathrm{mol})$ in chloroform.

For 3.4.2.2: $\quad$ 8\% weight loading of C26 TDE in liposome (actual: 5.4\%):

C26 TDE $(1.07 \mathrm{mg}, 0.976 \mu \mathrm{mol})$ dissolved in $1: 1 \mathrm{CHCl}_{3} / \mathrm{EtOH}$ was added to the mixture of PC $(8.07 \mathrm{mg}, 10.3 \mu \mathrm{mol})$ and cholesterol $(0.75 \mathrm{mg}, 1.94 \mu \mathrm{mol})$ in chloroform.

For 3.4.2.1 and 3.4.2.2: $\quad$ 16\% weight loading of C26 TDE in liposome (actual: $7.6 \%)$ :

C26 TDE $(2.146 \mathrm{mg}, 1.952 \mu \mathrm{mol})$ dissolved in $1: 1 \mathrm{CHCl}_{3} / \mathrm{EtOH}$ was added to the mixture of PC $(8.07 \mathrm{mg}, 10.3 \mu \mathrm{mol})$ and cholesterol $(0.75 \mathrm{mg}, 1.94 \mu \mathrm{mol})$ in chloroform. 
Quantification of TDE content in liposomes: The amount of C26 TDE incorporated into the liposomes was determined by measuring the carbohydrate content in aqueous soultion using the phenol-sulfuric acid method. ${ }^{122}$ The liposomes were pelleted by centrifugation at 10,000 RPM for 10 min and the PBS was removed using a glass pipette without disrupting the pellet. The liposomes were then resuspended in distilled water to a concentration of $0.5 \mathrm{mg} / \mathrm{mL}$, and titrated down in 2-fold dilutions in triplicates into a 96-well plate. The $50 \mu \mathrm{L}$ aliquot of a liposome suspension solution is mixed with 150 $\mu \mathrm{L}$ of concentrated sulfuric acid. Subsequently, $30 \mu \mathrm{L}$ of $5 \%$ aqueous solution of phenol is added rapidly to the mixture in a 96 well plate. After the plate is vortexed for $30 \mathrm{~s}$, the plate is placed for $10 \mathrm{~min}$ at room temperature in the dark for color development. Then, light absorption at $490 \mathrm{~nm}$ is recorded on a Titertek Multiscan microplate reader (Flow Laboratories, North Ryde, Austral). Reference solutions are prepared in an identical manner as above, except that the $50 \mu \mathrm{L}$ aliquot of liposomes is replaced with distilled water. The $5 \%$ phenol in water $(\mathrm{w} / \mathrm{w})$ was prepared immediately before the measurements. The standard curve of $\alpha, \alpha^{\prime}$-trehalose (in distilled water) was fitted with a line of best fit to give an equation, $y=-1.2171 x^{2}+1.4703 x-0.0097\left(R^{2}=0.99365\right)$. This equation allowed the corresponding amount of C26 TDE in the liposomes to be determined.

Measurement of liposome size: Size and size distribution measurements were performed in triplicate by dynamic light scattering (DLS) using a Zetasizer Nano ZS (Malvern Instruments Ltd, Malvern, Worcestershire, UK) at $25{ }^{\circ} \mathrm{C}$. Calculations of size distribution based on light scattering intensity were performed by the Zetasizer Nano ZS software from the correlation functions using the General Purpose algorithm (Malvern Dispersion Technology Software). Size measurements were made on the neat liposome suspension and also in samples, which were diluted by a factor 4 with a solution of PBS buffer before size measurements performed by DLS. The neat suspensions and dilutions were measured three times each. 
General methods and materials for biological assays: C57BL/6 mice and the Mincle $^{-/-}$mice were bred and housed in a conventional animal facility at the Malaghan Institute of Medical Research, Wellington, New Zealand. All animals used for the experiments were aged between 8-10 weeks. All synthetic compounds were confirmed to be free of endotoxin, at a sensitivity of $\leq 0.125 \mathrm{EU} / \mathrm{mL}$ by testing them in Limulus amebocyte lysate (LAL) assay using an endotoxin kit (Pyrotell, Limulus Amebocyte Lysate).

Generation of bone marrow derived macrophages (BMMs): Mice were culled in an enclosed $\mathrm{CO}_{2}$ chamber, and the legs and muscle were removed by dissection. Bone marrow cell suspensions were isolated by flushing femurs and tibias of 8- to 12-weekold C57BL/6 mice with Iscove's modified Dulbecco's medium (IMDM, Gibco) Aggregates were dislodged by gentle pipetting, and debris was removed by passaging the suspension through a $70 \mu \mathrm{m}$ nylon cell strainer. The cell suspension was spun in the centrifuge at $1000 \mathrm{RPM}$ for $10 \mathrm{~min}$, resuspended in $10 \mathrm{~mL}$ ACT buffer and incubated at $37{ }^{\circ} \mathrm{C}$ for $5 \mathrm{~min}$ to lyse red blood cells. After this time, $10 \mathrm{~mL}$ of IMDM was added to the suspension and the cells spun in the centrifuge at 1000 RPM for $10 \mathrm{~min}$. Cells were adjusted in IMDM medium supplemented with FBS (Gibco, 5\%), penicillin streptomycin (Gibco, 1\%) and 2-mercaptoethanol (Invitrogen, $55 \mu \mathrm{M}$ ) containing GMCSF (clone X63/GM-CSF murine cells, $10 \mathrm{ng} / \mathrm{mL}^{-1}$ ), to give a suspension of 250000 cells/mL, and seeded on 24-well plates. Cells were cultured in a humidified incubator at $37{ }^{\circ} \mathrm{C}\left(5 \% \mathrm{CO}_{2}\right)$ and the media changed on days 2,5 and 7 . On day 10 , the media was removed and the BMMs were either primed with IFN- $\gamma\left(10 \mathrm{ng} / \mathrm{mL}^{-1}\right.$, Peprotech) for $3 \mathrm{~h}$ prior to the addition of the compounds (Experiments described in section 2.4) or liposomes. 
BMM Assay: TDE and TME solutions were prepared $(2.5 \mathrm{mg} / \mathrm{mL}$ in PBS containing $2 \%$ DMSO), vortexed and warmed to $50{ }^{\circ} \mathrm{C}$ for $30 \mathrm{~min}$ ( $\mathrm{x} 3$ ) to ensure complete solubilisation of the compounds prior to administration to BMM cultures. BMM cultures were then treated with a known amount of TDE incorporated liposomes or 20 or $40 \mu \mathrm{g} / \mathrm{mL}$ of TDE, TME, with LPS (100 $\mathrm{ng} / \mathrm{mL})$ as a positive control and media only as a negative control for the times indicated. Supernatants were collected and tested immediately for $\mathrm{NO}$ levels or stored at $-80^{\circ} \mathrm{C}$ for subsequent cytokine analysis.

Quantification of NO: NO production in supernatants was evaluated by measuring the accumulation of NO in the culture medium by using Griess reagent (modified, Sigma Aldrich) containing $1 \%$ sulphanilamide in $5 \% \mathrm{H}_{3} \mathrm{PO}_{4}$ and a $0.1 \%$ solution of $\mathrm{N}-1$ naphthylethylene diamine hydrochloride. The mixture of Griess reagent and the culture supernatuant at a 1:1 ratio was incubated at $15 \mathrm{~min}$ at room temperature in the dark, and the optical denisty (OD) was determined at $550 \mathrm{~nm}$ with a plate reader. Liposometreated macrophage supernatants were collected at different time points. The culture supernatant were analysed for their NO contents by using the in the Griess assay. ${ }^{135}$

Statistical Analysis: All experiments were carried out three times, and only representative data are presented. All graphs and statistical analyses were generated in GraphPad Prism (GraphPad Software Inc., San Diego, CA, USA). A nonparametric unpaired $t$ test was used for analysis. Data was considered statistically significant when $p<0.05$. Error bars indicate \pm standard errors of the means (SEM). 


\section{REFERENCES}

(1) Linnebacher, M.; Maletzki, C.; Klier, U.; Klar, E. Langenbecks Arch. Surg. 2012, 397, 557.

(2) McCarthy, E. F. Iowa Orthop. J. 2006, 26, 154.

(3) Wiemann, B.; Starnes, C. O. Pharmacol. Ther. 1994, 64, 529.

(4) Zacharski, L. R.; Sukhatme, V. P. J. Thromb. Haemostasis 2005, 3, 424.

(5) Baban, C. K.; Cronin, M.; O'Hanlon, D.; O'Sullivan, G. C.; Tangney, M. Bioeng. Bugs. 2010, 1, 385.

(6) Detmer, A.; Glenting, J. Microb. Cell Fact. 2006, 5, 1.

(7) Calmette, A.; Guerin, C. Ann. Inst. Pasteur 1920, 34, 553.

(8) Qin, H. Y.; Sadelain, M. W.; Hitchon, C.; Lauzon, J.; Singh, B. J. Immunol. 1993, 150, 2072.

(9) Armentero, M.-T.; Levandis, G.; Nappi, G.; Bazzini, E.; Blandini, F. Neurobiol. Dis. 2006, 24, 492.

(10) Oscherwitz, J.; Hankenson, F. C.; Yu, F.; Cease, K. B. Vaccine 2006, 24, 3018.

(11) Schoenen, H.; Bodendorfer, B.; Hitchens, K.; Manzanero, S.; Werninghaus, K.; Nimmerjahn, F.; Agger, E. M.; Stenger, S.; Andersen, P.; Ruland, J.; Brown, G. D.; Wells, C.; Lang, R. J. Immunol. 2010, 184, 2756.

(12) Davidsen, J.; Rosenkrands, I.; Christensen, D.; Vangala, A.; Kirby, D.; Perrie, Y.; Agger, E. M.; Andersen, P. BBA. Biomembranes 2005, 1718, 22.

(13) Hunter, R. L.; Olsen, M. R.; Jagannath, C.; Actor, J. K. Ann. Clin. Lab.Sci. 2006, 36, 371.

(14) Khan, A. A.; Stocker, B. L.; Timmer, M. S. M. Carbohydr. Res. 2012, $356,25$.

(15) Bloch, H. J. Exp. Med. 1950, 91, 197.

(16) Lemaire, G.; Tenu, J. P.; Petit, J. F.; Lederer, E. Med. Res. Rev. 1986, 6, 243.

(17) Ryll, R.; Kumazawa, Y.; Yano, I. Microbiol. Immunol. 2001, 45, 801.

(18) Barry, C. E., III; Lee, R. E.; Mdluli, K.; Sampson, A. E.; Schroeder, B. G.; Slayden, R. A.; Yuan, Y. Prog. Lipid Res. 1998, 37, 143.

(19) Minnikin, D. E.; Polgar, N. Tetrahedron Lett. 1966, 2643.

(20) Watanabe, M.; Aoyagi, Y.; Ridell, M.; Minnikin, D. E. Microbiology 2001, 147, 1825.

(21) Penkov, S.; Mende, F.; Zagoriy, V.; Erkut, C.; Martin, R.; Paessler, U.; Schuhmann, K.; Schwudke, D.; Gruner, M.; Maentler, J.; ReichertMueller, T.; Shevchenko, A.; Knoelker, H.-J.; Kurzchalia, T. V. Angew. Chem. 2010, 49, 9430.

(22) Nishikawa, Y.; Katori, T.; Kukita, K.; Ikekawa, T. Nippon Kagaku Kaishi 1982, 1661. 
(23) Parant, M.; Audibert, F.; Parant, F.; Chedid, L.; Soler, E.; Polonsky, J.; Lederer, E. Infect. Immun. 1978, 20, 12.

(24) Toubiana, R.; Toubiana, M. J.; Das, B. C.; Richardson, A. C. Biochimie 1973, 55, 569.

(25) Polonsky, J.; Soler, E.; Varenne, J. Carbohydr. Res. 1978, 65, 295.

(26) Tocanne, J. F. Carbohydr. Res. 1975, 44, 301.

(27) Liav, A.; Goren, M. B. Chem. Phys. Lipids 1980, 27, 345.

(28) Khan, A. A.; Chee, S. H.; McLaughlin, R. J.; Harper, J. L.; Kamena, F.; Timmer, M. S. M.; Stocker, B. L. ChemBioChem 2011, 12, 2572.

(29) Lederer, E. Chem. Phys. Lipids 1976, 16, 91.

(30) Bottle, S.; Jenkins, I. D. J. Chem. Soc. Chem. Commun. 1984, 385.

(31) Jenkins, I. D.; Goren, M. B. Chem. Phys. Lipids 1986, 41, 225.

(32) Toubiana, R.; Das, B. C.; Defaye, J.; Mompon, B.; Toubiana, M. J. Carbohydr. Res. 1975, 44, 308.

(33) Johnson, D. A. Carbohydr. Res. 1992, 237, 313.

(34) Johnson, D. A.; Livesay, M. T. J. Carbohydr. Chem. 1998, 17, 969.

(35) Datta, A. K.; Takayama, K.; Nashed, M. A.; Anderson, L. Carbohydr. Res. 1991, 218, 95.

(36) Oosterom, M. W.-v.; Rantwijk, F. v.; Sheldon, R. A. Biotechnol. Bioeng. 1996, 49, 328.

(37) Park, O.-J.; Kim, D.-Y.; Dordick, J. S. Biotechnol. Bioeng. 2000, 70, 208.

(38) Olds, G. R.; Chedid, L.; Lederer, E.; Mahmoud, A. A. F. J. Infect. Dis. 1980, 141, 473.

(39) Yarkoni, E.; Bekierkunst, A. Infect. Immun. 1976, 14, 1125.

(40) Toubiana, R.; Ribi, E.; McLaughlin, C.; Strain, S. M. Cancer Immunol. Immunother. 1977, 2, 189.

(41) Ishii, F.; Takano, S.; Kohya, H.; Ishida, N. Gan to Kagaku Ryoho 1984, 11, 1629.

(42) Fujita, Y.; Okamoto, Y.; Uenishi, Y.; Sunagawa, M.; Uchiyama, T.; Yano, I. Microb. Pathog. 2007, 43, 10.

(43) Al Dulayymi, J. a. R.; Baird, M. S.; Roberts, E. Chem. Commun. 2003, 228.

(44) Al Dulayymi, J. a. R.; Baird, M. S.; Roberts, E. Tetrahedron 2005, 61, 11939.

(45) Al Dulayymi, J. a. R.; Baird, M. S.; Roberts, E.; Minnikin, D. E. Tetrahedron 2006, 62, 11867.

(46) Al Dulayymi, J. a. R.; Baird, M. S.; Maza-Iglesias, M.; Vander, B. S.; Grooten, J. Tetrahedron Lett. 2009, 50, 3702.

(47) Toschi, G.; Baird, M. S. Tetrahedron 2006, 62, 3221.

(48) Al-Dulayymi, J. R.; Baird, M. S.; Mohammed, H.; Roberts, E.; Clegg, W. Tetrahedron 2006, 62, 4851.

(49) Al Dulayymi, J. R.; Baird, M. S.; Roberts, E.; Deysel, M.; Verschoor, J. Tetrahedron 2007, 63, 2571.

(50) Koza, G.; Theunissen, C.; Al Dulayymi, J. R.; Baird, M. S. Tetrahedron 2009, 65, 10214.

(51) Grandjean, D.; Pale, P.; Chuche, J. Tetrahedron 1991, 47, 1215. 
(52) Nolibe, D.; Masse, R.; Tenu, J. P.; Lepoivre, M.; Petit, J. F. Cancer Immunol. Immunother. 1986, 23, 200.

(53) Holten-Andersen, L.; Doherty, T. M.; Korsholm, K. S.; Andersen, P. Infect. Immun. 2004, 72, 1608.

(54) Yamagami, H.; Matsumoto, T.; Fujiwara, N.; Arakawa, T.; Kaneda, K.; Yano, I.; Kobayashi, K. Infect. Immun. 2001, 69, 810.

(55) Saita, N.; Fujiwara, N.; Yano, I.; Soejima, K.; Kobayashi, K. Infect. Immun. 2000, 68, 5991.

(56) Castillo-Velazquez, U.; Aranday-Cortes, E.; Gutierrez-Pabello, J. A. Vet. Microbiol. 2011, 151, 51.

(57) Akira, S.; Uematsu, S.; Takeuchi, O. Cell 2006, 124, 783.

(58) Ishikawa, E.; Ishikawa, T.; Morita, Y. S.; Toyonaga, K.; Yamada, H.; Takeuchi, O.; Kinoshita, T.; Akira, S.; Yoshikai, Y.; Yamasaki, S. J. Exp. Med. 2009, 206, 2879.

(59) Werninghaus, K.; Babiak, A.; Gross, O.; Hoelscher, C.; Dietrich, H.; Agger, E. M.; Mages, J.; Mocsai, A.; Schoenen, H.; Finger, K.; Nimmerjahn, F.; Brown, G. D.; Kirschning, C.; Heit, A.; Andersen, P.; Wagner, H.; Ruland, J.; Lang, R. J. Exp. Med. 2009, $206,89$.

(60) Osorio, F.; Reis, e. S. C. Immunity 2011, 34, 651.

(61) Yamasaki, S.; Ishikawa, E.; Sakuma, M.; Hara, H.; Ogata, K.; Saito, T. Nat. Immunol. 2008, 9, 1179.

(62) Matsumoto, M.; Tanaka, T.; Kaisho, T.; Sanjo, H.; Copeland, N. G.; Gilbert, D. J.; Jenkins, N. A.; Akira, S. J. Immunol. 1999, 163, 5039.

(63) Miyake, Y.; Toyonaga, K.; Mori, D.; Kakuta, S.; Hoshino, Y.; Oyamada, A.; Yamada, H.; Ono, K.-i.; Suyama, M.; Iwakura, Y.; Yoshikai, Y.; Yamasaki, S. Immunity 2013, 38, 1050.

(64) Elass-Rochard, E.; Rombouts, Y.; Coddeville, B.; Maes, E.; Blervaque, R.; Hot, D.; Kremer, L.; Guérardel, Y. J. Biol. Chem. 2012, 287, 34432.

(65) Shenderov, K.; Barber, D. L.; Mayer-Barber, K. D.; Gurcha, S. S.; Jankovic, D.; Feng, C. G.; Oland, S.; Hieny, S.; Caspar, P.; Yamasaki, S.; Lin, X.; Ting, J. P. Y.; Trinchieri, G.; Besra, G. S.; Cerundolo, V.; Sher, A. J. Immunol. 2013, 190, 5722.

(66) Wells, C. A.; Salvage-Jones, J. A.; Li, X.; Hitchens, K.; Butcher, S.; Murray, R. Z.; Beckhouse, A. G.; Lo, Y.-L.-S.; Manzanero, S.; Cobbold, C.; Schroder, K.; Ma, B.; Orr, S.; Stewart, L.; Lebus, D.; Sobieszczuk, P.; Hume, D. A.; Stow, J.; Blanchard, H.; Ashman, R. B. J. Immunol. 2008, 180, 7404.

(67) Yamasaki, S.; Matsumoto, M.; Takeuchi, O.; Matsuzawa, T.; Ishikawa, E.; Sakuma, M.; Tateno, H.; Uno, J.; Hirabayashi, J.; Mikami, Y.; Takeda, K.; Akira, S.; Saito, T. Proc. Natl. Acad. Sci. U. S. A. 2009, 106, 1897.

(68) Matsunaga, I.; Moody, D. B. J. Exp. Med. 2009, 206, 2865.

(69) Feinberg, H.; Jegouzo, S. A. F.; Rowntree, T. J. W.; Guan, Y.; Brash, M. A.; Taylor, M. E.; Weis, W. I.; Drickamer, K. J. Biol. Chem. 2013, 288, 28457. 
(70) Furukawa, A.; Kamishikiryo, J.; Mori, D.; Toyonaga, K.; Okabe, Y.; Toji, A.; Kanda, R.; Miyake, Y.; Ose, T.; Yamasaki, S.; Maenaka, K. Proc. Natl. Acad. Sci. U. S. A. 2013, 1.

(71) Carletti, D.; Morais, d. F. D.; Gembre, A. F.; Masson, A. P.; Campos, L. W.; Leite, L. C. C.; Pires, A. R.; Lannes-Vieira, J.; Silva, C. L.; Bonato, V. L. D.; Horn, C. Clin. Vaccine Immunol. 2013, 20, 1162.

(72) Kamath, A. T.; Rochat, A.-F.; Christensen, D.; Agger, E. M.; Andersen, P.; Lambert, P.-H.; Siegrist, C.-A. PLoS One 2009, 4, 5771.

(73) Gram, G. J.; Karlsson, I.; Agger, E. M.; Andersen, P.; Fomsgaard, A. PLoS One 2009, 4, e6950

(74) Henriksen-Lacey, M.; Devitt, A.; Perrie, Y. J. Controlled Release 2011, 154, 131.

(75) Bangham, A. D.; Standish, M. M.; Watkins, J. C. J. Mol. Biol. 1965, 13, 238.

(76) Maurer, N.; Fenske, D. B.; Cullis, P. R. Expert Opin. Biol. Ther. 2001, 1, 923.

(77) Pinto-Alphandary, H.; Andremont, A.; Couvreur, P. Int. J. Antimicrob. Agents 2000, 13, 155.

(78) Watwe, R. M.; Bellare, J. R. Curr. Sci. 1995, 68, 715.

(79) Pagano, R. E.; Weinstein, J. N. Annu. Rev. Biophys. Bioeng. 1978, 7, 435.

(80) Latif, N.; Bachhawat, B. K. J. Biosci. 1984, 6, 491.

(81) van Rooijen, N.; van Nieuwmegen, R. Immunol. Commun. 1980, 9, 243.

(82) Lasic, D. D. Trends Biotechnol. 1998, 16, 307.

(83) Immordino, M. L.; Dosio, F.; Cattel, L. Int. J. Nanomed. 2006, 1, 297.

(84) Goyal, P.; Goyal, K.; Kumar, S. G. V.; Singh, A.; Katare, O. P.; Mishra, D. N. Acta Pharm. 2005, 55, 1.

(85) Zhang, Y. Preclinical Formulation 2008, 19.

(86) Milicic, A.; Kaur, R.; Reyes-Sandoval, A.; Tang, C.-K.; Honeycutt, J.; Perrie, Y.; Hill, A. V. S. PLoS One 2012, 7, e34255.

(87) Aderem, A.; Underhill, D. M. Annu. Rev. Immunol. 1999, 17, 593.

(88) Duffield, J. S. Clin. Sci. 2003, 104, 27.

(89) Murphy, K. T. P. W. M. J. C. Janeway's immunobiology; Garland Science: New York, 2012.

(90) Chellat, F.; Merhi, Y.; Moreau, A.; Yahia, L. H. Biomaterials 2005, 26, 7260 .

(91) Stout, R. D.; Watkins, S. K.; Suttles, J. J. Leukocyte Biol. 2009, 86, 1105.

(92) Sica, A.; Rubino, L.; Mancino, A.; Larghi, P.; Porta, C.; Rimoldi, M.; Solinas, G.; Locati, M.; Allavena, P.; Mantovani, A. Expert Opin. Ther. Targets 2007, 11, 1219.

(93) Bingle, L.; Brown, N. J.; Lewis, C. E. J. Pathol. 2002, 196, 254.

(94) Nitta, Y.; Sugita, T.; Ikuta, Y.; Murakami, T. Oncol. Res. 2000, 12, 25.

(95) Oh, Y.-K.; Nix, D. E.; Straubinger, R. M. Antimicrob. Agents Chemother. 1995, 39, 2104.

(96) van Rooijen, N.; van Kesteren-Hendrikx, E. Methods Enzymol. 2003, 373,3 . 
(97) Kelly, C.; Jefferies, C.; Cryan, S.-A. J. Drug Delivery 2011, 727241.

(98) Hiraoka, K.; Zenmyo, M.; Watari, K.; Iguchi, H.; Fotovati, A.; Kimura, Y. N.; Hosoi, F.; Shoda, T.; Nagata, K.; Osada, H.; Ono, M.; Kuwano, M. Cancer Sci. 2008, 99, 1595.

(99) Zeisberger, S. M.; Odermatt, B.; Marty, C.; Zehnder-Fjaellman, A. H. M.; Ballmer-Hofer, K.; Schwendener, R. A. Br. J. Cancer 2006, 95, 272.

(100) Diel, I. J.; Solomayer, E.-F.; Costa, S. D.; Gollan, C.; Goerner, R.; Wallwiener, D.; Kaufmann, M.; Bastert, G. N. Engl. J. Med. 1998, 339, 357.

(101) Ahsan, F.; Rivas, I. P.; Khan, M. A.; Torres, S. A. I. J. Controlled Release 2002, 79, 29.

(102) Malam, Y.; Loizidou, M.; Seifalian, A. M. Trends Pharmacol. Sci. 2009, $30,592$.

(103) Csuk, R.; Schulthei ü, A.; Sommerwerk, S.; Kluge, R. Tetrahedron Lett. 2013, 54, 2274.

(104) Woudenberg-van Oosterom, M.; van Rantwijk, F.; Sheldon, R. A. Biotechnol. Bioeng. 1996, 49, 328.

(105) Tsuzuki, W.; Kitamura, Y.; Suzuki, T.; Kobayashi, S. Biotechnol. Bioeng. 1999, 64, 267.

(106) Schiefelbein, L.; Keller, M.; Weissmann, F.; Luber, M.; Bracher, F.; Friess, W. Eur. J. Pharm. Biopharm. 2010, 76, 342.

(107) Neises, B.; Steglich, W. Angew. Chem. 1978, 17, 522.

(108) Twibanire, J.-d. Ä. K.; Grindley, T. B. Org. Lett. 2011, 13, 2988.

(109) Paul, N. K.; Twibanire, J.-d. Ä. K.; Grindley, T. B. J. Org. Chem. 2012, $78,363$.

(110) Liav, A.; Goren, M. B. Carbohydr. Res. 1986, 155, 229.

(111) Datta, A. K.; Takayama, K.; Nashed, M. A.; Anderson, L. Carbohydr. Res. 1991, 218, 95.

(112) Nishizawa, M.; Yamamoto, H.; Imagawa, H.; Barbier-Chassefière, V.; Petit, E.; Azuma, I.; Papy-Garcia, D. J. Org. Chem. 2007, 72, 1627.

(113) DeTar, D. F.; Silverstein, R. J. Am. Chem. Soc. 1966, 88, 1013.

(114) Upadhyaya, S. P.; Davis, F. S.; Lee, J. J.; Zaw, K.; Bauer, L.; Heimer, N. E. J. Heterocycl. Chem. 1997, 34, 1607.

(115) Zhang, X.; Goncalves, R.; Mosser, D. M. In Curr. Protoc. Immunol. John Wiley \& Sons, Inc.: 2001.

(116) Auwerx, J. Experientia 1991, 47, 22.

(117) Austin, P. E.; McCulloch, E. A.; Till, J. E. J. Cell. Physiol. 1971, 77, 121.

(118) Stocker, B. L.; Khan, A. A.; Chee, S. H.; Kamena, F.; Timmer, M. S. M. ChemBioChem 2014, 15, 382.

(119) Stuehr, D. J.; Nathan, C. F. J. Exp. Med. 1989, 169, 1543.

(120) Lang, R. Front. Immunol. 2013, 4.

(121) White, K. L.; Rades, T.; Furneaux, R. H.; Tyler, P. C.; Hook, S. J. Pharm. Pharmacol. 2006, 58, 729.

(122) Masuko, T.; Minami, A.; Iwasaki, N.; Majima, T.; Nishimura, S.-I.; Lee, Y. C. Anal. Biochem. 2005, 339, 69. 
(123) Bryan, M. C.; Plettenburg, O.; Sears, P.; Wong, C.-H. Chem. Biol. 2002, 9, 713.

(124) Malvern Instruments Ltd 2004. Zetasizer Nano Series User Manual

(125) Bassal, S.; Liu, Y. S.; Thomas, R. J. S.; Phillips, W. A. Biochim. Biophys. Acta. 1997, 1355, 343.

(126) Grando, F. C. C.; Felicio, C. A.; Twardowschy, A.; Paula, F. M.; Batista, V. G.; Fernandes, L. C.; Curi, R.; Nishiyama, A. Braz. J. Med. Biol. Res. 2009, 42, 599.

(127) Ghosh, J.; Guha, R.; Das, S.; Roy, S. Infect. Immun. 2014, 82, 607.

(128) Konig, S.; Regen, T.; Dittmann, K.; Engelke, M.; Wienands, J.; Schwendener, R.; Hanisch, U. K.; Pukrop, T.; Hahn, H. Cancer. Immunol. Immunother. 2013, 62, 1587.

(129) Richardson, M. B.; Williams, S. J. Front. Immunol. 2014, 5.

(130) Yamasaki, S. Eur. J. Immunol. 2013, 43, 3156.

(131) Lobato-Pascual, A.; Saether, P. C.; Fossum, S.; Dissen, E.; Daws, M. R. Eur. J. Immunol. 2013, 43, 3167.

(132) Kodar, K.; Timmer, M. S. M.; Stocker, B. L. Unpublished results.

(133) Li, Z.; Mintzer, E.; Bittman, R. J. Org. Chem. 2006, 71, 1718.

(134) Khan, D. R.; Rezler, E. M.; Lauer-Fields, J.; Fields, G. B. Chem. Biol. Drug. Des. 2008, 71, 3.

(135) Sun, J.; Zhang, X.; Broderick, M.; Fein, H. Sensors 2003, 3, 276. 